\title{
570.945 P764v
}

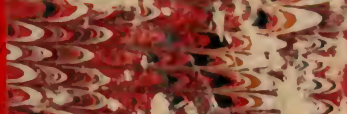
s. (4)

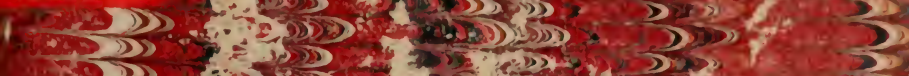

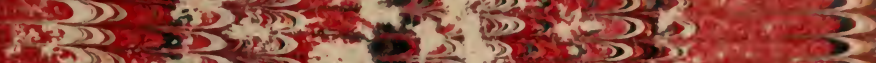

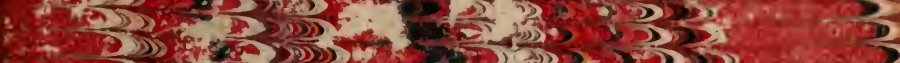

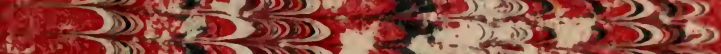

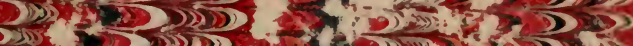

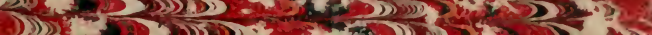

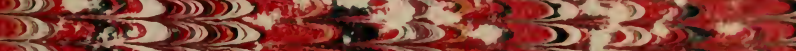

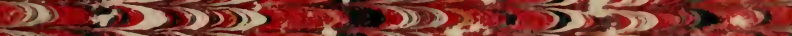

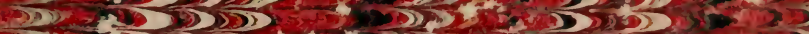

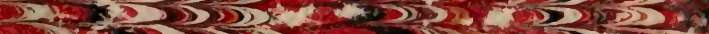

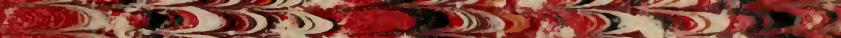
W.

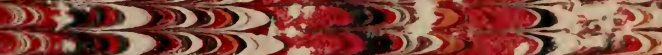

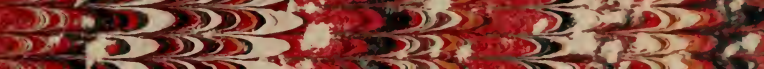

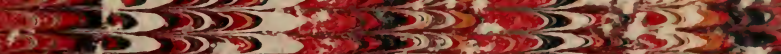

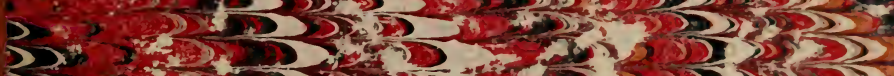

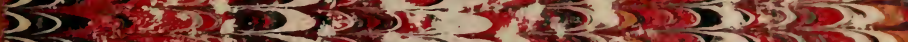

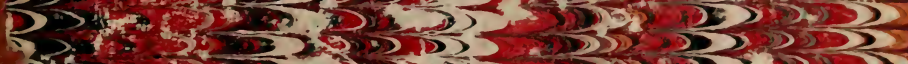

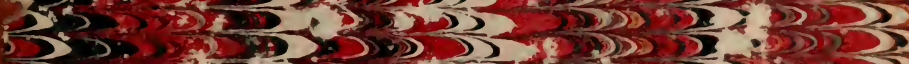

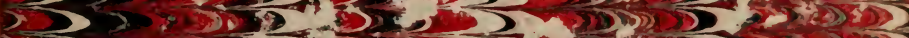

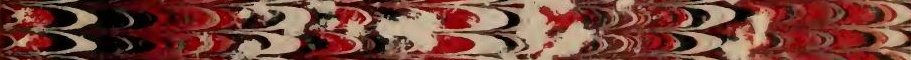

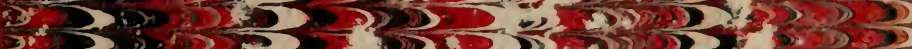

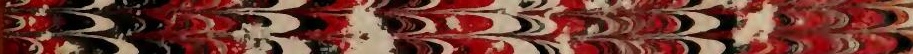

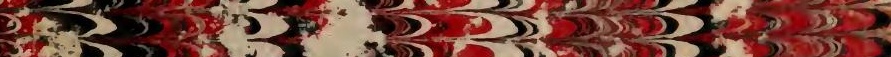

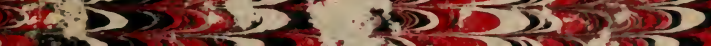

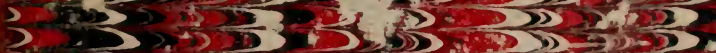

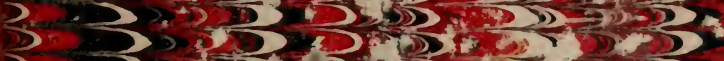

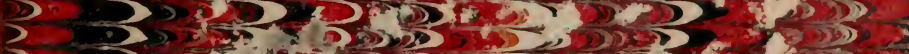

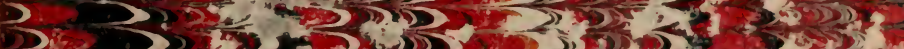

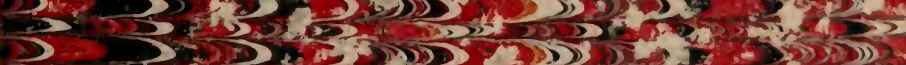

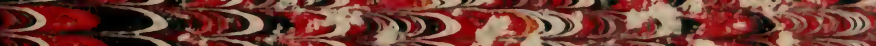

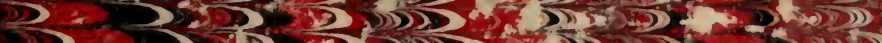

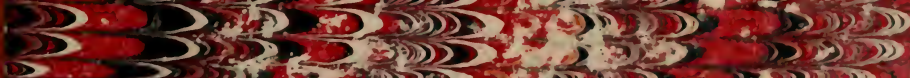

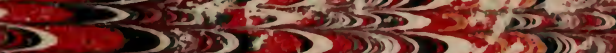

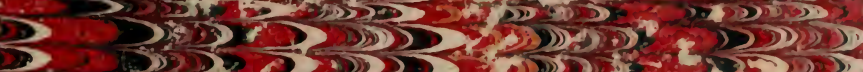

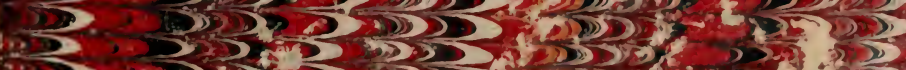

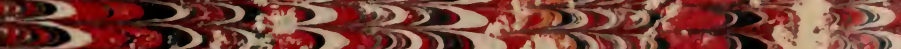

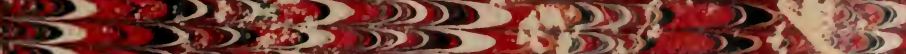

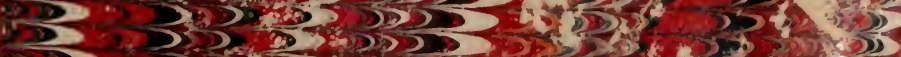

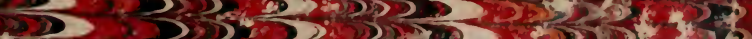



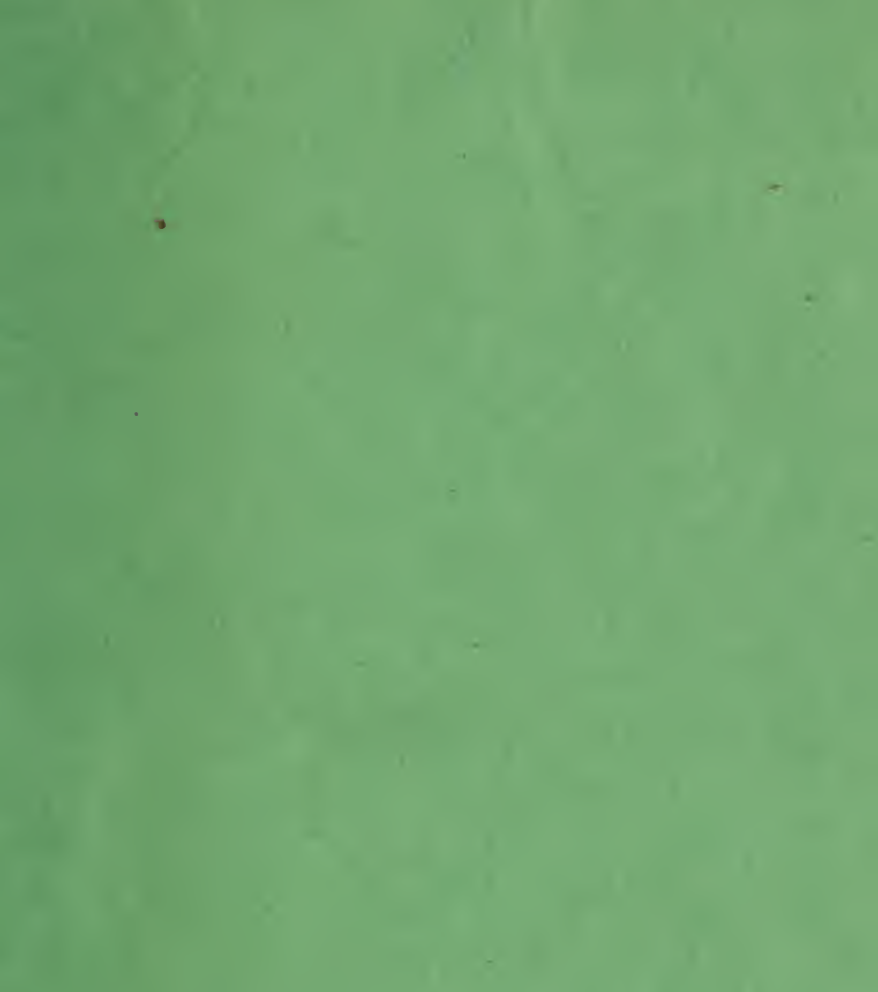

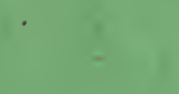

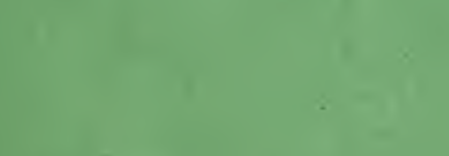






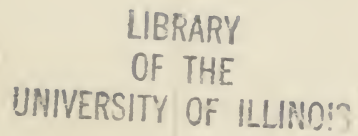




\section{F. I}

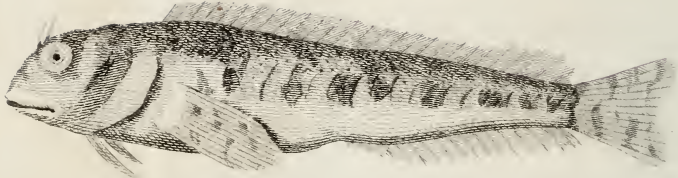

\section{F. II}

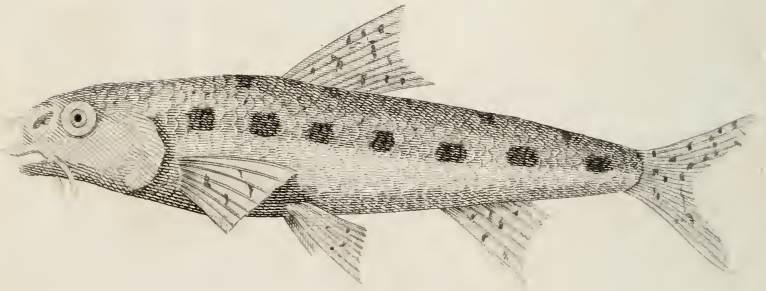




\section{I A G G IO}

A L L A G, O D, I G R D A E

\section{AL MONTE BALDO}

I I C U I SI RAGIOIN

DELLE COSE NATURALI DI QUEI LUOGHI

$$
\text { A G GIUNTOVI UN CENNO }
$$

SULLE CURIOSITA'DEL BOLGA

$$
\text { E DEGLI ALTRI MONTI VERONESI }
$$

$\begin{array}{llllllllllllll}D & \text { I } & \text { C } & \text { I } & \text { R } & \text { O } & \text { P } & \text { O } & \text { L } & \text { L } & \text { I } & \text { N } & \text { I }\end{array}$

$$
\text { DOTTORE IN MEDICIMA E CHIRURGIA }
$$

$$
\text { con una' } T \text { avola in rame }
$$

\section{IN VERONA}


.......... Vieni qui dove

Tra'l marmifero Torri, e la pescosa Torbole, Re de gli altri altero monte, La soggetta Malcesine, l'amena Primogenita sua Baldo vagheggia. Spolverini Riseid. Lib. I. 


\section{A L S I G. C URZIO SPRE NGE L}

PROFESSORE DI MEDICINA E DI BOTANICA NELL'UNIVERSITA' DI HALA IN SASSONIA

Lettera 1.: Sul Lago di Garda

Q uello, che da assai tempo io vi ò promesso, eccovi finalmente, o mio celebre amico. Vi esporrò adunque ciò, che vidi al Benaco e al monte Baldo nelle frequenti gite, che feci in que'luoghi deliziosi veramente ed ameni sopra il credere di chi non li vide. Che se avverrà, che dal mio dire vi venga alcun diletto, infinito ve ne verrebbe certamente, dove vi piacesse scorgere per gli occhi vostri ciò, che ora scorgete per le parole altrui. Le quali e sia per colpa del dicitore, e sia per diffetto di linguaggio mal giungono ad esprimere in tutto quel che nell' anima si sente.

Ora perchè l'ultima volta appunto, ch'io fui in que' luoghi, percorsi l'intera circonferenza del Lago, e visitai in ogni sua parte monte Baldo, ò in pensiero di descrivervi tale ultima gita; la quale non vi so dire quanto mi riuscisse deliziosa e cara. Imperocchè alla sempre nuova dolcezza della cosa s'aggiungeva la dolcezza dell' amicizia .

Di già nello scorso inverno io e tre miei amici amatori dell'istoria naturale avevamo divisato, come prima la stagione il permettesse, recarsi a diporto insieme e ad istruzione al vaghissimo Lago di Garda. E venuto il tempo di mandare ad effetto il proponimento, e già essendo noi acconci alla partenza, oh diss'io, 
sarebbe pur vergogna per giovani naturalisti solcar l'onde del Benaco, e non salir monte Baldo, i cui piedi bagnano l'onde stesse. Dico adunque doversi a un tempo ascendere la montagna, che voi sapete essere carissima agli amatori della Botanica. Piacque a tutti la proposta : però quando sgombre si videro di neve l'alte vette del monte, ci ponemmo in cammino. Era il solstizio d'estate, e partimmo sull'alba da Verona, e per una piacevole via tra colle e colle arrivammo a Bardolino, villaggio amenissimo sulle sponde del Lago . Il Lago di ch'io vi favello stendesi da settentrione a mezzo giorno pel tratto di circa trenta cinque miglia (I), e giace tra le fauci dell' alpi retiche, le quali a mano a mano declinando l'abbracciano da Riva che n'è capo, fino a Peschiera che n'è l'estremità. Meno di tre miglia largo da prima vassi ampliando si, che da Torri a Maderno à incirca sette miglia, e infine da Salò a Garda non è minore di dodici. La sua superficie s'innalza sopra quella dell' Adriatico metri 77. 82. (2). La sua profondità varia senza fine: la maggiore è a settentrione poco lungi da Campione

(1) Miglia comuni, vale a dire di metri 1780,80 ciascuno, e però minori del miglio geografico di 60 al grado, che consta di metri $185 \mathrm{r}, 85$, e incirca tre quarti maggiori del miglio nuovo o chilometro ch'è di rooo.

(2) L'osservazionc barometrica fu da me istituita a Garda il di 22 Luglio 1815 alle 9 e mezzo antimeridiane, mentre l'atmosfera era placidissima, e serenissimo il cielo. Il barometro segnava 27. I r. 13. 16.mi; il termometro all' aria libera all' ombra 18 gradi sopra zero. Secondo la misura presa pure barometricamente dagli ufficiali francesi del genio, e inserita nel Manuel toposraphique militaire stampato a Parigi, la superficie del Lago s'innalza dal mave metri ior. 2. Non debbo però tacere che da una livellazione eseguita da ingegnere veronese si trae, che la superficie del Lago a Lazise è più bassa forse venti metri del pelo dell'acqua dell' Adige a Verona, il qualc è più alto della superficie dell' Adriatico di m. 67.07 . 
sotto la Madonna di Monte Castello, e cid ch'è singolare a pochi passi dalla rupe, che fa parete al Lago, ove arriva a centottanta metri, laddove nel mezzo la profondità maggiore è di cento trenta (1).

Al cominciar della state, gonfiatisi i fiumi, che metton foce nel Lago, s'innalzano le acque a più d'un metro, ed iscono in varj luoghi del bacino: a tale epoca la navigazione è ben sovente pericolosa. Veggonsi dal suo seno sorgere tre vaghe isolette (2), e vaghissima è poscia la penisola Sermione, che s'avanza fra le limpide onde del Lago. Metton foce in esso il fiume Sarca a settentrione tra monte Breonio e Peneo, e il Ponale, e la Brasa, e Tusculano, ed altri piccoli torrenti a ponente. A Peschiera n'esce il Mincio. E muovon poi le acque lel Lago due venti periodici, che spirano la più parte dell' anno, e che sono sommamente favorevoli alla navigazione (3) : perocchè

(I) Secondo il Conte Bettoni la profondità maggiore, ch' è tra Castelletto e Gargnano, aggiunge a 584 metri.

(2) La prima e maggiore dicesi l'Isola de' Frati, perchè eravi ai tempi passati un convento di Francescani minori. $\dot{E}$ posta tra Saló e Dcsenzano quasi di fronte a S. Vigilio. Si congiunge alla prossima spiaggia di Manerbe per una catena di scogli subacquei. La 2.da è Tremollone lunghesso la sponda veronese tra Brenzone e Malcesine. La 3.za è uno scoglio detto l'Isoletto presso Malcesine.

(3) Il commercio del Lago è vivissimo, e ben maggiore sarebbe dove si rendesse navigabile il Mincio. Le più grosse barche portano incirca 600 miriagrammi e sono poche. La loro grandezza va diminuendo sino ai battelli di pescatore, sui quali si caricano roo miriagrammi, o dieci persone con due o tre harcajoli. Tutte le barche vanno a remo o a vela, e quando non trae vento scorrono terra terra coll' ajuto $\mathrm{de}^{\prime}$ remi. Sono munite d'un'ampia vela cuadrata maneggiata col mezzo di un albero sostenuto da corde, e dirette da un timone. Esse non navigano mai contro vento. Le più grosse con vento favorevole percorrono fino a dieci miglia all'ora : un battello di pescatore a quattro remi fa fino cinque iniglia, dove il Lago non sia contrario. 
uno vien da tramontana, e spira da intorno alla mezzanotte al mezzodi, l'altro trae dall'austro, e dura dal mezzodì a sera (I). Ed è quel primo sì violento, che concilia al Lago quell'aspetto tanto minaccioso, e si lo sconvolge, che

\section{Col fremito dell' onde al mar s'agguaglia (2).}

Però la direzione di tali venti viene in più luoghi cambiata dalle fauci de' monti, che sboccano verso il Lago, onde si destano varie correnti .

Temperata e purissima è l'aria, leggerissime e limpidissime sono le acque. Ma come dipinger le sponde? Che non fece la natura, onde compreso fosse lo spettatore d'infinito diletto, di stupore infinito? Imperocchè gli orridi dirupi del settentrione, e le balze spaventose mutansi presto nel piegare al meriggio in ridenti pendici, in collinette coperte di gelsi, d'olivi, di viti, e de' più cari doni del pomifero autunno . Apparisce novellamente la scena solitaria ed aspra, e siamo a nuove rupi, a massi ignudi e minaccianti, al silenzio, all' orrore, a cui succede di nuovo il gajo aspetto della verzura e de' dolci colli. Nè si può dire, di che incanto sieno $i$ giardini vaghissimi, che adornan quei lidi, dove la fragranza de' cedri e degli aranci ne rimembra $i$ tanto decantati da poeti d'Alcinoo e delle Esperidi.

A Bardolino dimorammo alcuni di presso uno de' nostri compagni, e furono veramente deliziosi : bello il cielo, tranquillo il Lago, su cui in leggerissima bar-

(1) Paŗe cDsa ragionevole ripetere la cagione di tal fenomeno dal movimento del sole, e l'esame della posizione del Lago e de' monti e dell'alpi prossime sembra farla manifesta.

(2) Fluctibus, et fremitu assurgens, Benace, marino. Virg. Georg. Ir. 
chetta scorrevam volando. Da Bardolino avviandoci a settentrione venimmo a Garda. Da questa terra prende ora nome il Lago un tempo Benaco. Giace nel fondo d'ampio seno, e sulla vetta d'una rupe perpendicolare all'onde eravi a' tempi andati una rocca. Ora è luogo di cara solitudine, alla quale si giunge per un sentier tortuoso fiancheggiato da annosi cipressi,

$$
\text { che fanno al sacro albergo }
$$

Di triste e pur soavi ombre corona (I).

Dall'alto dell'eremo scorgesi piacevolmente da un lato il Lago, dall' altro la ferace valle di Caprino .

Poc'oltre a Garda s' incontra il promontorio di S. Vigilio, che è veramente il più ameno luogo della sponda veronese. Imperocchè posto a mezzodi, e difeso a settentrione da monte Baldo, che ivi comincia ad erger le sue cime, si puó dire che goda una perenne primavera. Crescon ivi a cielo aperto piante delle più calde regioni, e fiorisce tra le fessure degli scogli l'Agave americana. I colli all' intorno son tutti coperti di robusti olivi, di viti, e d'alberi fruttiferi, e qui colgonsi in primavera ottimi fichi, i quali resisterono al rigore dell'inverno. L' arte poi s'aggiunse alla felicità della natura, e sulla sommità dello scoglio che sporge sul Lago collocò un vago giardino, tutto di cedri ed aranci odoroso, ed alto e magnifico s'innalza un palagio, d'onde l'occhio vagheggia l'intero Lago, e l'opposta sponda bresciana. Ora seguendo il cammino incontriam varj villaggi. Sono essi Torri, Castelletto, Brenzone;, e Malcesine, ch'è di tutti principale. Oltre al qual luogo restringesi il Lago assai; non

(1) Poesie canpestri d'Ippolito Pindexoxtz. 


\section{8}

$v^{\top}$ àn più colli, e ignudo apparisce da una banda, ed orrido il torreggiante fianco di Baldo, dall'altra stanno. gli inospiti e inaccessibili monti bresciani. Qui cupo è il Lago e melanconico. Ma vaghissima è la sponda settentrionale. Fra Torbole e Riva vedesi serpeggiante scorrere per angusta valle, e sboccar nel Lago il fiume Sarca, e amenissime colline s' elevan all' intorno, che a mano a mano più alzandosi fansi selvose; quindi son fine a tutto l'altissime alpi eternamente nevose.

Passammo adunque alla sponda oocidentale, la quale è veramente più della veronese deliziosa. Posta tra levante e mezzogiorno gode $d$ ' una temperatura sommamente dolce, oltremodo favorevole alla vegetazione, e alla salute, e al buon umor degli uomini . La spiaggia è cospersa di giardini bellissimi, da cui esce olezzo soavissimo di cedri e di aranci. E fra le prime cose ond'avemmo a meravigliare si fu la caduta del Ponale, che dagli alti dirupi gittasi con tre passi nel Lago . Rumoreggian l'onde, che si spezzano, e gli spruzzi di contro al sole d'ogni più vago modo si dipingono. Scopresi quindi fra le ignude balze la romita piaggia di Limone non meno che altra amena, e appresso la piccola di Campione. Ma scena giocondissima n'offerse l'ampio seno di Gargnano, sicchè ogni altro luogo a noi parve men bello : e colline qui vedi e pendici ridenti di prati e d'erbe odorifere, e d'olivi e d'alberi fruttiferi coperte, e di leandri e d'allori : tra case e palagi superbi s'ammirano i più leggiadri giardini di aranci e di cedri, e dell'olezzo e fragranza, che da essi ne venì, tutto pieno. In seguito visitammo Tusculano rinomatissima per le sue cartiere fabbricate sul fiume dello stesso nome, del pari che per le fucine di ferro ivi condotto dalle 
miniere delle valli vicine. La feracità e amenità di questi luoghi avventurosi trasse per avventura anzi ogn'altro gli uomini ad abitarlo fin da tempi i più rimoti . Vuolsi che quivi fosse l'antica Benaco, che diè il nome al Lago, perduta dicono per tremuoto, e secondo altri per innondazione arrenuta al principio dell' era nostra . Ed oltre le belle iscrizioni da noi qui vedute non meno che nelle prossime terre ( $\mathrm{r}$ ), altro monumento credette taluno di scoprire sotto l'onde, le reliquie di quella città. La di lei esistenza però reputano altri favolosa,

(r) Tali iscrizioni furono raccolte dal Panvinio, Grattarolo, Scipione Maffei e da altri. Le due seguenti, che non ò veduto ne' tre citati Scrittori, non fia sdicevole di qui addurre.

In un antico marmo situato nel territorio di Piva presso il tempio di S. Cassiano:

$$
\begin{aligned}
& \text { L. MAG. H'CIATO } \\
& \text { CL. SEVERA . MIARITO } \\
& \text { KARISSIMO . ET CORN } \\
& \text { VALERYO . FILIO . PIEN'TIS } \\
& \text { SIMO E ET . MAG. PRIS } \\
& \text { NIANO . SOCERO, B. M. } \\
& \text { ET SIBI . ET } \equiv \equiv \text { I. MENO } \\
& \text { RIAM . CO } \equiv \equiv \mathrm{ET} \text {. SUI . COLL. } \\
& \text { N. B. AD. ROSAS. ET PRO } \\
& \text { FVSIONES . Q. A. F. A. C. } \\
& \text { H.S. N. LX. HIL. NEDIT . }
\end{aligned}
$$

Alla Pieve vecchia di Manerbe in valle Tenesi in un angolo del Campanile sopra un cippo di marmo bianco ò veduto la seguente iscrizione a bei earatteri e bene scolpita, ma colla dimensione delle lettere decrescente ad ogni linea da ro centimetri ai $\{$.

$$
\begin{aligned}
& \text { C. LVCRETIVS } \\
& \text { CLERASIVS }
\end{aligned}
$$

SEX. VIR. AVG. PRIX.

ET. TRIDENT. GRAT. SIBI

ET COMIN. ONESIME

VXORI . CARISSIMAE

C. IVVCRETIO . HER METI

ALVMINO . PIISSINI.

LIBERTIS. LIBERTABVSOYE. E. T. 
e l'antico nome amano derivare dalla piccola terra di Naco situata alle radici del monte Peneo. Ma lasciando tai cose dico, che abbandonato Tusculano tosto ne si fe' incontro $\mathrm{Maderno}$. Fummo poscia al ridente seno di Salò, tutto cinto $d^{\prime}$ amenissime e fruttifere colline, e saluta Gazano patria di Bonfadio infelice, e veduto Manerbe, o com' altri vuole Minerva, giungemmo a Desenzano terra grossa e fiorente per commercio, quindi a Rivoltella. Ma a sè ne traea la venusta Sermione, dove tra le rovine e le antiche volte ancor ne parve risonasse la voce del tenero cantore di Lesbia. Vedemmo l'ittiogena Peschiera, e l'origine del Mincio, nè dimenticato Lazise ci riducemmo a Bardolino.

$\mathrm{V}^{\prime}$ ò dipinto del modo, che meglio ò saputo, le bellezze del Lago. Voglio ora soddisfare alla vostra dotta curiosità, e vi dirò quel che mi venne fatto di scorgere rispetto a storia naturale.

Per ciò che spetta alla costituzione delle sponde, la veronese formata in gran parte da monte Baldo consta al par di questo di una calcare stratificata di color bigio perlato o bianchiccio, e talora incarnato, a frattura liscia e terrosa, piena di petrificazioni marine. Mancano peró quivi le rocce trappiche. Tutta la falda di Baldo è sparsa di ciottoli porfirici e granitici smussati e rotondati dalle acque. La curiosità più singolare, che dassi a vedere in questa sponda, è il marmo giallo di Torri di varie tinte, fra le quali pregevolissimi sono il marmo azzurro, e il giallo con ammoniti spatificate. Le cave esistono in copia sulla pendice di monte Baldo, sovente ascoste sotto un' argilla schistosa o ardesia di color rosso, e frammiste a filoni perpendicolari di ematite di ferro, e a varj strati di marmo bianchissimo e 
variopinto in rosso. I colli poi, che dalle radici di monte Baldo vanno declinando fino a Peschiera, sono nella massima parte terreni d'alluvione, constando ora di arena ammonticchiata, ora di breccia composta di sassi calcari porfirici e granitici impastati da cemento calcare.

I monti della spiaggia settentrionale e di quella da ponente constano al pari della veronese di calcare stratificata, o di marna calcare con frequenti vestigia di corpi organizzati marini petrefatti, come Veneri, Mitili, ed altre conchiglie bivalvi, Corni d'ammone, Retipore, Madrepore, Echini, ecc., e frammiste a pietra focaja ora in massi, ora a piccoli strati e di molte fatte, massime verso Gargnano, dove oltre la focaja comune rossiccia rinviensi la bigia, la gialla nereggiante, e l'azzurra, e la verde, talora insieme connesse, ed ora più ora meno trasparenti. La massa calcare il più è bianca o rossiccia; ma a Gargnano, Campione, Salò, e all' Isula de' Frati presenta varie gradazioni di colori, dal cinerino all' azzurro e al nero, e riceve anche pulimento, siccome il vago marmo nero di Tremosine. Questa fatta di calce colorata stroppicciata manda un odore fetente. Nella calce e marna azzurrognola dei monti di Campione sono racchiuse palle più o meno voluminose di silice sovente coperte d'un astuccio calcare più duro della roccia, simili a quelle rinvenute dal Prof. Maironi da Ponte nel monte Misma del Bergamasco, e dal Prof. Catullo nel monte Carrera del Bellunese. Alcune di tali palle però constano interamente di calce o di marna indurita. I colli Salodiani sono a luogo a luogo di terza formazione. Tutti poi sono cosparsi di cristalli di quarzo in prismi bellissimi e di ciottoli di diaspro giallo e rosso ed al- 
tre tinte, non che di calcedonie, agate, e corniole eleganti, e petroselci squamosi il più rossicci.

L' arena della spiaggia è costituita di ciottoli calcari dagli adjacenti monti caduti, frammisti a sassi e frammenti angolosi granitici e porfirici bellissimi di molte fatte, e di schisto in ispecie, cui le acque de' torrenti ànno staccato dalle rocce, che costituiscono $\mathrm{gl}^{\prime}$ interni monti bresciani. Imperocchè ivi esistono ampi tratti formati di schisto argilloso, cui talora è sovrapposta la calcaria, e le serve di base un'arenaria rossa o grauvachia composta di frammenti più o meno voluminosi di quarzo impastati in un cemento argillo-ferruginoso rossiccio, e mescolato di squamette di mica. Lo schisto micaceo è all' arenaria ben sovente sottoposto, e tal rara volta sorrapposto. Fra le fenditure degli strati poi apparisce lo spato calcare in prismi esagoni elegantissimi. Entro a tali rocce stanno pure le rinomate miniere di ferro, che lavoransi nelle fucine fabbricate sui fiumi Brasa e Tusculano. Sul margine meridionale finalmente del Lago scorgonsi quıa e là piccole stratificazioni di torba.

Un fenomeno singolare noto da lunghissimo tempo offre il Lago al lato orientale di Sermione. Osservando attentamente la sua superficie scopresi una congerie non interrotta di bolle d'aria talora fumanti, che in cinque distinte parti escono gorgogliando sulla sopraffaccia dalla profondità d'incirca 70 metri. Una sesta sorgente apparisce più addentro nel Lago. L'odore di tali holle è d' nova fracide inclinante al sulfureo, il sapore acidetto . Alcuni sperimenti ànno svelato in esse gas acido carbonico e gas idrogeno solforato. 
Altro fenomeno, di cui mi fecero accorto i pescatori, è una corrente subacquea da essi detta il corrivo, la quale quando esiste sconvolge e ammucchia loro di tal fatta le reti, che sono astretti a desistere dalla pescagione . Questa ò veduto a Lazise, a Bardolino, a Garda, ma assai entro il Lago: rapidissima poi è a $\mathrm{S}$. Vigilio, a Torri, Malcesine, Limone, Campione, Gargnano. Essa à due direzioni, o da mezzodi a settentrione, o da settentrione verso austro. La superficie del Lago suvente non dà verun indizio dell' esistenza del fenomeno, mentre o è placida, o è appena lievemente increspata, ovvero le sue onde sono agitate in senso opposto. Continua talora due o tre giorni, e persevera nell'incominciata direzione. Secondo quello che affermano i pescatori suole apparire il corrivo dopo grandi burrasche. Le due prime volte appunto, ch'io osservai il fenomeno, il di e la notte antecedente il sovero ovvero tramontano avea sconvolto l'onde benacensi, e la direzione era da mezzogiorno a settentrione. Una terza volta vidi la corrente in direzione opposta, e nel giorro antecedente fuvvi un fiero turbine cagionato dall' óra o vento meridionale. Dal che ne emerge, che la cagione di un tal fenomeno sono i venti, i quali sospingendo violentemente le onde ora a una estremità del Lago ora all'al tra, le costringono a schiudersi una corrente opposta negli strati inferiori onde ristabilire l' equilibrio . Che quello che asseveriamo sia vero, il conferma l'osservazione di ciò che interviene nella parte meridionale del Lago, come a Bardolino, Lazise, Maderno, Tusculano . Quivi il bacino essendo molto ampio, e potendo le acque rifluire liberamente, la corrente subacquea e di gran lunga meno rapida che a S. Vigilio, a Torri, e nelle 
parti più settentrionali. Oltracciò, avendo quivi frequente dominio altri venti oltre $\mathbf{i}$ due principali, assume la corrente altre direzioni, vale a dire da oriente verso occidente, o da occidente, in oriente secondo che il vento preceduto muoveva da occidente ovvero da oriente.

I vegetabili che crescono nelle acque del Lago sono pressochè tutti quelli che si rinvengono ne'luoghi acquatici e palustri. Però fra le fenditure delle rupi germogliano varie piante delle regioni australi. Eccovi il catalogo delle principaii che ò rinvenuto e nelle acque e sulle sponde, aggiunto alle meno comuni il luogo dove nascono.

Agave americana. Nelle rupi a S. Vigilio, Limone, Gurgnano, Tusculano, Salò, Isola dei frati.

- B. fol. margine luteo. $S$.

Vigilio.

Agrimonia Eupatorium.

Agrostis miliacea. Canpione, Gargnano.

Alisma Plantago.

Allium angulosum. Torri.

- neapolitanum. Bardolino.

— paniculatum.

- rotundum. S. Vigilio,

Moniga .

Althaea officinalis .

Alyssum calycinum .

Amaranthns Blitum

-

- - sylvestris.

Anagallis tenella. Garda .

Andropagon Gryllus

- halepensis

- Hischoemum .

Anethum Fœniculum.Nelle rupi. Anthemis arvensis

- Cota

- tinctoria .

Anthericum ramosum.
Apargia incana. Ponale, Campione, $S$. Vigilio.

Arabis Turritis. Isola dei Frati, Sermione.

Arenaria bavarica. Riva, Limone, Carnpione. Artemisia Absynthium .

- - camphorata.

Arum italicum. Garda, Minerva. Arundo Donax . Gargnano, Salò, Isola dei Frati.

_- epigejos. Limone. Phragmites.

Asclepias Vincetoxicuu .

Asparagus acutifolius. Garda, Bardolino .

Aspidium Thelipteris.Peschiera. Aster Amellus

- - annuus.

Astragalis Onobrychis. S.Vigilio, Gargnano.

Athamanta Cervaria.

- Libanotis

- Oreoselinum.

Atriplex patula. Peschiera. Atropa Belladonna.Tra Sermione e Peschiera.

Betonica officinalis,

Bidens bipinnata. 
Bidens cernua. Peschiera. - tripartita.

Bromus erectus. Huds. Smich. Buphtalmum grandiflorum

\section{—- Speciosissimum . Presso} Tremosine.

B. caule plerumque monanthodio, foliis alternis, ex amplexante basi ovatis, nudis, denticulatis, subciliatis, inferioribus in petiolum alatum semivaginantem desinentibus . Mihi.

Provenit etiam in monte Grigna vallis Saxina provincia Comensis, loco dicto la valle dei molini. Corollulæ luteæ. Quintili et Sextili mensibns floret. Perenn.

Buphtalmum spinosum. Brenzone, Riva.

Bupleurum Odontites. Castelletto.

\section{Gerardi .}

Challitriche aquatica, $\propto$ autumnalis. Peschiera.

- $y$. C. verna.

Calluna vulgaris.

Caltha palustris. Peschiera.

Campanula bononiensis. Tra

Castelletto e Torri.

- glomerata

- patula. Brenzone, Ca-

stelletto.

__ persicifolia

- rotundifolia. Malcesine,

Ponale.

-

- spicata

- Tracheliunn.

Carduus defloratus.

Carex acuta

—_ alba. Malcesine.

- baldensis. Limone.

clandestina. Malcesine. dioica. Malcesine.

- Michelii

- paludosa.
Carex pendula. Peschisra, Tusculuno .

_- pillulifera

- recurva

- Schreberi

- verna

- vulpina.

Carpesium cernuum. Isola dei Frati, Rivoltella, Peschiera.

Caucalis grandiflora.

Caulinia fragilis

Celtis australis.

Centaurea crupina

* - Jacea, ß. C. amara. L. WV .

- n nigrescens

- paniculata

- Scabiosa.

Ceratophyllum demersum. $P e$ schiera, Sermione.

Cercis Siliquastrum .

Circæa lutetiana .

Chara hispida

- vulgaris.

Cheirauthus erysimoides.

Cnicus palustris. Peschiera, Tusculano.

Cochlearia Coronopus. Salò. Conjugata angulata. Vauch.

—_ cruciata Ejusd. Nei prati, _- lutescens Ejusd. e nei se_ porticalisEjusd. ni, e nei __ princeps Ejusd.) ruscelli.

Convolvulus Cantabrica .

Coriandrum testiculatum .

Cornus mascula .

Coronilla Emerus.

Coryllus Avellana .

Cratægus monogyna .

Crepis foetida.

Cucubalus Behen.

Otites .

Cyclamen europæum .

Cyperus flavescens

- fuscus

- glomeratus. W. Enum. non Sp. pl. Ejusd. (Segu. Ver. III. p. 68. t. 2. f. 3.) Peschicra, Lazise. 
Cyperus longus?

- Monti.

Cytisus argenteus. Gargnano, Salò

- hirsutus

- nigricans

- purpureus. Malcesine, IVavené, Riva.

- sessilifolius.

Daphne alpina. In campagnola di Malcesine fra le rupi del. la sponda.

- Laureola. Lungo il $P_{0}$ nale, Campione.

Dianthus atro-rubeus.
Erythrea Centauriun. E. caule tetragono dichotomo corynboso, foliis ellipticis trinerviis, floribns sessilibus, calycibus quinquefidis, corollæ tubo dimidio brevioribus. Mihi. Copiose circa Piscariam, Malamscilicem et alibi. Ann.

* - intermedia. Mihi. Chironia intermedia. Mérat Nouv. flor. de Paris pag. 9 . E. caule ramoso dichotomo, compresso-tetragono, foliis ovatis, summis linearibus, floribus pednnculatis, calycibus quinquepartitis corolla tubo subæqualibus. Mihi. Provenit in pratis humidis secus lacum in loco dicto $\mathrm{Lu}$ gana Sirmionem inter et Arelicam. Augusti initio florentem legebam. Mihi occurrit etiam secus Ticinum fluvium; nec non adthermas Aponinas in colle dicto il Montiron, post Junii mensis initium florens. Ann.

Eupatorium cannabinum .

Euphorbia Lathyris. Alla Pieve vecchia di Minerva in valle Tenesi, presso l'antica iscrizione superiormente recata.

- nicæensis

- palustris. Sermione, $\mathrm{Pe}_{\mathbf{C}}$ schiera, Lugana.

Nell'opera citata ò creato il genere Hexagonia con un funEuphrasia tricuspidata. Canpione, Ponal, Malcesine.

Eronimus latifolius. Malcesine.

* Favolus mori. Mihi. Hexagonia mori. Pollin. Hort. et prov. veron. pl. nov. vel min. cogn. pag. 35. fig. 2. Malcesine, Bardolino, Salò. go, cui non seppi riferire a nessuno degli ammessi dal Persoon nella sua celebre Synopsis methodica Fungortum. Ora trovo che il Sig. Beauvois nella Flora dell' Owar e Benin à stabilito il genere Favolus, che à appunto i caratteri della mia Esagonia. Addotto pertanto un tal genere come al mio anteriore, e ad esso riferisco come nuova specie la mia $\mathrm{He}$ xagonia mori. 
Ferula nodiflora. ( ardia.

Festucat fluitans. Pesclierele, Tusculano.

—— serotinat.

licus Carica .

Fontina!is antip: a . $P_{e}$ schiera.

Fumaria capreola Nei giardini di Limon

Falium Bocconei

- svlvaticum.

Geranium robertianum.

firatiola officinali Peschiera Hellehorus foctidt. A Moniga

presso Manerbe.

- niger. Punale, Campione.

- viridis. Gaidu.

Hesperis tristis. Limone.

Hieracium florentilum

- sabaudım

- n umbellatum.

Hippuris vulgaris. Peschiera.

Holtonia palustris. Pardolino. Hydrocharis Morsus ranx.Peschicia.

Hydrodiction pentagonum . Vanch. Peschiera.

Hypericum humifusum . Garda. - quadrangulum. Peschiera. IIypnum riparioides. Tusculano. Jasionc montana.

Ilex Aquifolium. Campinne e altrove.

_ ß. inermis. Varicta bellissima, presso il Ponale.

Inula britannica

- dysenterica

- hirta

- Pulicaria

- squarrosa

- germanica .

Iris Pseudo-Acorus. Peschicra, Sermione. Juncus articulatus

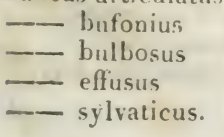

Peschiera.

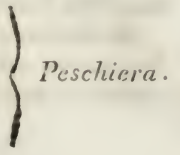

Jungermannia helveticat. Alle cateratte del Ponale.

\section{Lactuca perennis}

—_ Scariola.

Laserpitum Siler. Monce Castello, Ponule.

Lathyrus hirsutus. Peschierce. - sylvestris. Campione, Salio.

Laurus nobilis.

Lavandula Spica .

Lecrsia oryzoides. Pesclicera,

Lemna minor

- polyrhiza

- trisulea.

I.cpidium graminifolium

- potræum. Malcesine.

Linkia Nostoc

* - pulposa. Mihi.

- verrucosa. Bardolino.

Lonicera Xylosteum .

Lotus siliquosus. Pescliera, Sermione.

Lycopus curoprus

- exaltatus. Lagana, Pcscliera.

Lythrum Salicaria.

Morsilea quadrifolia Pescliera.

Medicago Gerardi.

Melanipyruu sylvaticum .

Melica cœrulea .

Melilotus officinalis

- - - $\beta$. fl. albo.

Melissa officinalis.

Melittis Melissophyllum

Mentha aquatica

—— arvensis Peschier,

_- Pulesium (e altrove.

Menianthes Nymphoides. I'e schicra.

Mespylus Cotoneaster. Punale, Monte Casteillo.

Myriophyllum spicatum
- verticillalum.

Najas monospermid.

Nepetat nuda. 
Nerium Oleander. Campione, Limone, Salu, Moniga, Castelletto.

Olea europæa. Fra le rupi.

Ononis minutissima. Monte Castello, Gargnano. pinguis.

Onosma echioides .

Ophrys apifera. Alla Rocea di Garda.

___ arachniles. Ivi .

- - aranifera. S. Vigilio.

Orchis hircina. S. Vigilio.

- Morio

- piramidalis.

Origanum vulgare.

Pastinaca sativa.

Pedicularis palustris. Peschiera.

Phalaris arundinacea.

Phillyræa latifolia. S. Vigilio, Monle Castello, $e$ altrove.

Phytolacca decandira.

Picris hieracioides.

Pimpinella dioica.

Pistacia Terebintlius.

Plantago subulata.

Poa aquatica. Peschiera.

* - - $\beta$. prolifera. Varietà bellissima, presso $P e-$ schiera.

- compressa.

Polygonum amphybinm. Peschiera, Sermione, Tusculano.

- Hydropiper

- minus

- Persicaria.

Polysperma glomerata. Vauch.

Potamogeton com-

pressum

- crispnm

- densutu

- lucens

- marinum

- natars

- perfoliatum

- serratum.

Potentilla alba. Garda, Bar-

seni .
Potentilla caulescens. Riva,

Campione, Gargnano.

Prenanthes muralis.

Primula veris. Ma!cesine.

—— vulgaris.

Prunella graudiflora.

Pteris ayuilina.

Punica Granatum.

Pyrus forminalis.

Quercus Esculus

- Ilex

- Iles

- pedunculata. Salio, Desenzano, Rivollella.

_- Robur.

Ranunculus fluviaitis \} Pesclice- scelleratus. $\{r a$.

Pieseda Phyteuma. Bardolino, Garda.

Rihamnus Frangula

_- pumilus. Monte $\mathrm{Ca}$ stello.

- saxatilis.

Rhus Cotynus.

Riccia fluitans. Peschiera.

Rosa rubiginosa fl. albo. Campione.

Rosmarinus officinalis. Copio sissimo fra le fessure di Monte Castello, e presso Cannpione.

Pubus coesius. Pesctriera.

Rumex acquaticus.

Ruscus aculeatus.

Puta chalcpensis.

Saccharum Ravenna. Peschierce e lungo la Sarca.

Sagittaria sagitaifolia . Peschiera.

Salix caprea

Nei — riparia

porti, triandra.

Salvia glutinosa.

e nei Salvinia natans. Peschiera.

Samolus Valerandi. Peschiera,

Bardolino, Tusculano.

Saponaria officinalis .

Satureja hortensis. Garda.

dolino, Lazise. 
Satureja montana.

Scabiosa graminifolia. Limone, Mome Castello, Gargnano.

- Succisa. Peschiera, Cavalcaselle.

- - transilvanica. Peschicra.

Schonus Mariscus. Peschiera.

- - nigricans. Peschiera, Malcesine.

Scirpus acicularis. Peschiera. - Caricis. Peschiera, Lugana.

- - dichotomus. Presso Manerbe .

Senccio aquaticus: foliis inferioribus obovatis subdentatis, reliquis pinnatifido-lyratis dentatis, radio patente, seminibus glabris. Mihi. Nei luoghi umidi.

- Jacıbra : foliis radicalibus petiolatis ellipticis dentatis, caulinıs imis pinnatifido-lyratis, reliquis scmiamplexicaulibus pinnatifidis, radio patente, seminibus hirsutis. Mihi. Nelle selvette dei colli a Castelletto, e in valle della Sarca.

- paludosus

__ viscosus. Malcesine, Torbole.

Sesleria coerulea.

Silene nutans

- Saxifraga. Limone, S. $V$ igilio .

Sisymbrium amphibium

- Nasturtium

- - sylvestre

- tenuifolium.

Sium angustifolium

- latifolinm.

Solanum Dulcamara

- miniatum $\alpha$ fructu miniato

- $-\beta$. fructu luteo.

Salò .

- nigrum.

Sonchus palustris . Peschiera.

Sparganium ramosum .
Scirpus Itoloschonus. Peschicril.

- lacustris

- maritimus

mucronatus

- triqueter.

Scrophularia aquatica. Peschiera, Sermione.

- nodosa.

Scutcllaria galcriculata .

Seseli anuuum. Burdolino, Colia.

la Sarca.

Sempervivum tectorum. Monte Castello.
Spartium junceum. Campione, Gargnano.

_ radiatum. Campione, Manerbe.

Stachys palustris

- sylvatica.

Stipa pennata. Garda.

Tamus communis.

'leucrium Chamædrys

- montanum .

Thalictrnm angustifolium

- flavum.

Thrincia hirta. Peschiera.

Thymus Acynos

_- angustifolius

- Calamintha

- grandiflorus

- lanuginosus

- Nepeta.

'Tordylium maximum. Bardolino, Salò. 
Trichodium caninum.

'Trifolium angustifolium. Peschierce.

- fragiferum

__ medium

- montanum

- rubeus

- scabrum. S. Vigilio.

Turritis hirsuta.

Tussilago Farfara. Peschiera. - Petasites .

Typha angustifolia

_- latifolia

- minima.

Ulva gelatinosa. Vuuch. Púschiera.

* - Lurbinata. Milii. Sermionc, Isola dei frati. Vedrassi la descrizione inferiormente.

Utricularia vulgaris. Peschiera. Valeriana dioica. Peschiera, Bardolino.

_- officinalis.

Molte e squisite specie di pesci annidano nel Benaco, parte d'acqua dolce, parte originarj del mare; infra $\mathrm{i}$ quali merita particolare menzione il carpione. Crescono quivi i pesci a mole meravigliosa, e sono saporitissimi . Quelli da noi osservati sono i seguenti :
Nome scientifico
CONDROTTERIGJ
Nome v'ernacolo

l'etromyzon branchialis $A P^{\prime} O D I$

Muræna Anguilla no.

Viburuum Lantana

- Opulus. Burdolino, SerVicia lutea. Malcesine, GaiErncenos

\section{Vitis vinifera.}

Zyzyphus Paliurus

_- vulgaris.

di Gaida, fra le fenditure della rupe.

Vallisneria spiralis.

Verbascum Blattaria

Lychnitis
Verbeua officinalis.
Veronica Anagallis
- Beccabunga
_- oflicinalis. Ponale, Cam-
pione, Malcesine.
_- prostrata

Lychnitis
Verbeua officinalis.
Veronica Anagallis
- Beccabunga
- oflicinalis. Ponale, Cam-
pione, Malcesine.
- prostrata

Lychnitis
Verbeua officinalis.
Veronica Anagallis
- Beccabunga
_- oflicinalis. Ponale, Cam-
pione, Malcesine.
_- prostrata

Lychnitis
Verbeua officinalis.
Veronica Anagallis
- Beccabunga
_- oflicinalis. Ponale, Cam-
pione, Malcesine.
_- prostrata

Lychnitis
-_ - . fl. albo.
Verbeva officinalis.
Veronica Anagallis
_- Beccabunga
_- oflicinalis. Ponale, Cam-
pione, Malcesine.
- prostrata

Valeriana rubra 
Caput conicum obtusum; mandibule dentibus minutis acuris armate, superiore longiore; oculi rolundi, pupilla nigra, irtde argentea vel citrina; branchiarum opercula mollia; dorsum convexum; linea lateralis recta; ahromen alhicans; anus in medio corporis; pinua dorsalis, caudilıs, et pectorales cinereat, fuscomaculatie, analis et ventrales hyalinie.

\section{Yome scienlifico \\ Nome volguie}

\section{TORACICI}

Cottus Gobio ADDOMINALI

Cobitis Barbatula

$$
-
$$

Salmo Trutta

- Umbla.

\section{Esox Jucius}

Clupea Alosa major

- parvula

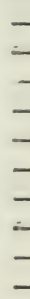
- Carpio

- Tinca

- Idlus

- Phoxinus

- Grislagine

- Ruttilus

- Aphya

- Orfus

- Alburnus -

- Vimba

- benacensis. Mihi. (fig. 2 ) -

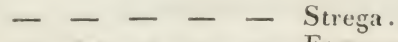

C. pinna ani radiis 7 , cirris 2 , cauda bifida. D. ro-11. P. 8. V. 8. A. 7. C. $18-19$.

Non è questo il Temolo dei popoli d'Italia, Salmo Thymallus, il quale abita nel nostro Adige, nel Ticino, e in altri nostri fumi, ma è un pesciolino della lunghezza al più di otto centimetri (incirca un palmo), di un sapore dilicatissimo, non molto comune, e che si suole pescare solo nella stagione iemale. Appartiene ai Ciprini barbati, come quello ch'è fornito di due cirri alla bocca; à dieci o undici raggi alla pinna dorsale, otto o nove alle pettorali, otto alle 
ventrali, sette all'anale, diciotto, o diciannove alla coda. A' il capo conico con due fossette bislunghe nella fronte avanti agli occhj, l'iride argentina? la mandibola inferiore appena un po' più breve della superiore; i coperchi delle branchie lisci; la linea laterale dritta; il corpo coperto di squame poco distinte, d'un colore cinereo lucido con macchie oscure; le pinne cinerine con punti e striscie fosche.

Prima di chindere questo articolo piacemi farvi un cenno d'un pesce della famiglia de' toracici, il quale comunque non si rinvenga nel Benaco, pure non vuole essere negletto, perchè da niuno, a qnello ch'io sappia, venne ricordato come abitatore de' nostri fiumi . E questo il Pleuronectes flesus, figurato dal Block nella tav. 44 , e definito dal Linneo nella Fn. svedese nel seguente modo, $P$. oculis dextris, linea laterali aspera, spinulis ad pinnas. D. 55.62. P. 10-12. V. 6. A. 40.41. 43. C. 14.17 . Se ne pesca di varia grandezza in primavera, nella state, e in autunno ne' fiumicelli Tartaro e Molinella, il primo de' quali comunica col Po, e il secondo mette nel primo. Ora sapendo noi, come il Pleuronette fleso, o Passero fluviatile soglia in primavera montare dal mare nella foce de' fiumi, onde deporre le uova nell' acque dolci, potrebbe credersi originario del1'Adriatico. Ma può essere eziandio, che abiti costantemente, e si moltiplichi nei suddetti luoghi, mentre vive bene nell' acque dolci, ed è noto, che gli abitatori della Frisia l'ànno trasportato ne' loro stagni, e reso famigliare .

Oltre del Gambero comune, Cancer Astacus, rinvengonsi al nostro Lago due granchietti . L' uno è il Cancer Squilla (Gamberozoli volg.), che abita infra l'erbe 
palustri tanto del Benaco, quanto delle risaje nostre, ed è la varietà a rostro dritto. L'altro è il Cuncer Pulex (Salterello volg.) ; ritrovasi nel greto a quattro dita, dove si moltiplica prodigiosamente. Dalla sua bocc a esce un umore corrosivo, atto a sciogliere la terra. E poichè nelle ore calde suole escire dal covacciolo, reca sommo danno alle tele di lino e di canape, che si stendono dai benacensi ad asciugare ed imbiancare sulla spiaggia, mentre le foracchia di mille modi con l' umore onde si prepara l'alimento. Fu scoperto anche in alcuni pozzi di Verona, e nelle terme di Caldiero .

Io vorrei tesservi un intero catalogo degli augelli, che frequentano il Benaco, molti de' quali rinvengonsi pure nelle nostre risaje ; ma finora non fu possibile istituire un distinto esame di tutti. Quelli che da noi furono esaminati e preparati in diverse occasioni sono $\mathrm{i}$ seguenti :

Nome scientifico.

Nome italico e volgare.

\section{OCH E}

Anas Cygnus

- Boscas

Clangula

- clypeata

- Fuligula

- Penelope

- strepera

- rufina -

—_ acuta - ferina -

- Crecca

\section{- africana}

- Querquedula

- fusca.

Mergus Serrator --

- Albellus

Pelecanus Onocrotalus

Colymbus glacialis

- Immer

- cornutus
- Ciguo. Ilal. Veron.

- Anitra selvatica. Ital. Veron.

- Quattr' occhi. Ital. Veron.

- Anitra longirostra.

- Cápo negro. Venez.

- Anitra Penelope. Ital.

- Anitra strepera.

- Capo rosso maggiore. Ital.

- Coda lancea. Ital.

- Collo rosso. Ital.

- Garganello, Sartella, Cercevolo. Iual. Mazzurino. $V$ er.

- Anitra africana .

- Cercedula, Gargauello, Sartella . Ital.

- Anitra nera. Ilal.

- Smergo segatore. Ital. Serula. Venez.

- Smergo bianco. Ital.

-- Pelicano comune od Onocrotalo. Ilal.

- Colimbo ghiaccialc.

- Margou. Benac.

- Colimbo cornuto. 
Colymluis cristatus

- auritus

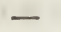

- Urinator

- minor.

Larus hybernus Sterna minuta

__ fissipes

- hyrundo -

$$
\text { GRALLL }
$$

Fulica atra - - chluropus. Ardea stellaris -

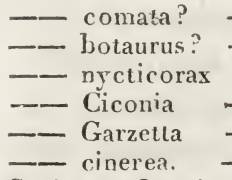
Scolopax Glottis .
- Colimbo crestulo o Sperga. Tlal. Struffon dalla cresta. Benac.

- Colimbo orecchiuto. maggiore. Struffoni Benue. Fisanelle, Compita. Venez

- Colimbo minore. Ital. Strufloni. Benac.

- Coccai, Ziveltinc, Sardenar. Verun.

- Gavia marina. Ital.

- Rondione marino. Itat.

- Sterna rondine. Ital.

- Folaga nera. Ilal. Folega. Veron.

asa Folagr verdipiede.

- Il Trombone o la Trabucine'. Flal Tarabello. Veron.

- Airone. Teron.

- Airone. Veron.

- Airone. Veron.

- Cicogna. Itul. Zigogna. I'eron.

- Garzelta. V'eron.

- La Sgarza o Airone cinerino. Ttal. - Totane, Limosa. Venez.

Ma poichè io v'ò fatto parola degli augelli, che visi-r tano l'onde del Benaco, piacciavi che v'adduca il nome pare de' principali fra quelli che abitano o passano pei colli benacensi, e per gli altri monti veronesi, o che frequentano le risaje nostre e i luoghi palustri $\left(^{*}\right)$. Perö. rispetto ai nomi vernacoli debbo avvertire darsi il caso, non a vero dire frequente, che tale augello sia chiamato con diverso nome nelle diverse parti della Provincia, e avvien pure che lo stesso norne diasi a più specie congeneri. Una tale considerazione mi fia per avventura scudo contro la taccia d'inesatto nomenclatore.

$$
\text { SPARVIERI }
$$

Falco Milvus - - Nibbio. Ftal. Milan. Veron.

$$
\begin{gathered}
\text { - Buteo - Pojana o Falcone Bozzago. Ital. Poja- } \\
\text { na, Puja. Veron. }
\end{gathered}
$$

${ }^{*}$ Q Questi c i superiormente accennati formano parte d' una raccolta d'augelli imbalsamati da uno di noi, quattro anni fanno, presentata a S. A. il Principe Eugexio, e che fu collocata nella villa Bonaparte a Milano. 
Falco communis - - Falcone. Tlat:

- - var. italiea - Falcone. Ital.

- Nisus - - S - Slarvierr comune, o Fringucllajo. Ital.

balchetto. I eron.

Tinnunculus - Comibello, Gavincllo, Tristarcllo. Ilul.

Gavinel. Veron.

palumbarius. - Astore, T'erzolo. Ital.

Strix Bubo - - - Dugo, Guflo reale o baubagianui selvistico. It. Gran duca. Fer.

- passerina - Ciretta. Ic. Zivetta. f'eron.

- Hainmea - - Allocco minore. JiOtns. - - Duca cornuto, Allocchercllo, Assiuolo. Il. Ciedo? Veron.

Lanius Excubitor - Velia maggiore o cinerina, Gazzasparviera. It. Regestola, Regestola falconera. Feron.

-Collurio. - - Velia maggiore ferruginosa, Passera gazzera, Falconello. ílal.

Corvus Corax - - Corvo comume. Il. Veron.

Corone - - Cornaechia comune. Ical.

Cornix - Cornaeclia ammantata o Meflacelia. Io

Monedula - - Taccola. Ital. Íeron.

Pyrrhocerax - 'Tacrola, Monlan. veron.

- Glandarius - - La Ghiandaja o Gazza ghiandaja. It. Gazza. V $\boldsymbol{c} r$.

- Gazzera comume o Gazza Il. Checca V $\mathrm{T}$ s.

- Corvo franginoce.

- Coraccia garrula; Gazza marina, Pica marina. Itul.

Oriolus Gralbula.

Cuculus canorus.

Yunx Torquilla.

Picus viridis

- major - - Picchio vario o Culo rosso.It. Pico.V er.

- medius. - - Pigozzo o Culo rosso, Pipo. Ilal. Pico. $\mathrm{Ver}$.

Sita curopar. - - Peciotto comunc. It. Pariacciac. Pence.

Alcedo Ispida. - - Uccello pescatore, Martino pescatore. Jual. Pionbini. Veron.

Ierops Apiaster.

Upupa Epops. -

Certhia muraria faniliaris. -

- Dareno o Apiastro comune. Ital.

- Bubbula. It. Galletto di mar. Benac. Gallo del paradiso. Venez.

- Cerziar murajola. Il.

- Cerria comune o Rampichino. Tlat. Rampeghino. fier. 
GRALLE

Fulica nævia

- - Grinetta, Tordo geisemino. It. Scorzana, Sinarziana. Mantov. Grugnetto. Novar.

fusca - - Giratola, Viatara.Ver. Grugnetto . Novara.

- B. Gallinula maj. Giratola. Ver.

Rallus Crex - - - Scopajola, o il Re di Quaglie, o la Gallinetta terrestre. Ital. Re de Quaje. Ver.

_- aquaticus - - Scorzana, Squarziana. Veron. Manlov. Porzana, Forzana. Venez.

- Porzana. - - Girardine. Veron. Milan. Novar. Porzana. Bologn.

Ardea Gru . - - Gru. Ital. Veron.

Recurvirostra Avocelta - Avocetta.

Tringa pugnax - - Il Combattente. Ilal.

- Vanellus - - Pavoncella. Ilal. Paonzina. Veron.

Scolopax rusticula - Beccaccia comune, Gallinella. Ital. Beccassa. Ver. Arzia. Bresc. Gallinazza. Lomb.

- Gallinago - - Beccaccino reale, o la Pizzardella. Ital. Beccanotto. Veron.

- arcuata - - Torquato. Ital. Arcuato. Venez.

Charadrius Pluvialis. - Piviero, Pluviero. Ital. $\mathrm{Ver}$.

$$
\text { GALLINE }
$$

Tetr $\geqslant 0$ Coturnix

- Perdix

- rufus - =

- Francolinus -

- Urogallus -

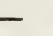

-

Quaglia. Ital. $\mathrm{Ver}$.

Pernice. It. Ver. Pernigone. Lomb.

- Coturnice. Ital. Coturno. Veron.

- Francolino. Ilal.

- Gallo cedrone, o Gallo alpestre maggiore, od Urogallo. Ital Gallo de montagna, Gallo salvadego, Zedron. Veron. Ven.

- Lagopus - - Pernice bianca di monte, o Pernice alpestre, o Francolino di monte. Ital. Gallinette, Galline selvadeghe. Ver. Pernise bianche di montagna . Ver. Trentin.

_- Tetrix. - Crallo alpestre minore, o Faggiano nero. It. Gallo de montagna. Benac.

PAsseri.

Columba Oenas -

- Turtur - -

Alauda arvensis -

- pratensis -
- Piccione terrajolo. It. Favazzo. Ver.

- Tortora, Tortoro. It. Ver.

- Allodola maggiore o Panterana. Ital. Lodola . Ver.

- Allodola pratajola, o la Mattolina. Ital. Scozzetta, Sgussetta. Ver. 
Alauda pratensis $\beta$. Alau-

da pratensis candida- Sguzzetta hianca. Fet

- campestris - Spipoletta. Firent. Sguzzellone. Ver. Tordino. Ven.

--- trivialis - -- -- Allodola triviale. It. Lodola, Tordina. Ver.

cristata -- - -- Allodola cappelluta. It.Cappellota. Ver.

Calandra - -- -- Calaudra. Ital. Ver.

arborea. - - - Allodola arborea, Tottovilla. Ital.

Sturnus vulgaris -- -- Storno. It. Ver. Storliuo. Ver. Stornello. Venez.

--- cinglus -- -- -- Storno acquajuolo. Itul. Mcrlo aquarolo. $V \mathrm{cr}$.

Turdus viscivorus - -- Tordo maggiore. It. Stordella. Benac. Dresso . Lombard.

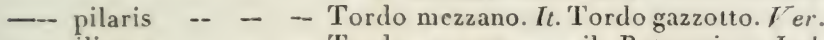

-- iliacus -- -- -- Tordo comune o il Boltaccio. Iial. Tordo zigarol. Benac.

musicus - -- -- 'Tordo, 'Tordo sassello o il Malvizzo . Ital. Tordo. Veron.

Merula -- -- -- Merlo. Ital. Ver.

-- Merlo rosso, Storno rosso. Ital. Ver.

-- Squarozzolon. Ver.

-- Passera solitaria. Ital. Ver.

-- Garrulo di Boemia. Ital. Galletto di busco. Ital. Ver.

Loxia Curvirostra -- Il Crociere. Ital. Becco in crose. Ver. --- Coccothraustes - Il Frosone o Frisone. Ital. Ver. Sfrisone. Bresc.

--- Pyrrhula -- -- Monacchino o Ciuffolotto. Ital. Zionzolo, Ziolunzolo. Veron.

--- Chloris. -- -- Verdone. Ital. Zaranto, Taranzolo, Teranzo. Veron.

Emberiza Hortulana - Ortolano. Ital. Ver.

nivalis - -- -- Ortolano nevale o di montagna. Ital.

Osel de la neve. Benac.

Citrinella -- -- Zivolo giallo . Ital. Smajarda. Ver. Spajarda. Mov.

passerina -- -- Pionza. Ver.

Cia. -- -- -- Cia. Llal. Zia, Ziga. Ver. Lumbard.

Fringilla cœlebs -- -- Fringuello. It. Franguello, Finco. Fer.

--- Montifringilla - Fringuello montanino, o la Peppola. Itul. Miontano. Ver.

Carduelis -- -- Cardello, Calderuggio, Cardellino. It. Gardelin. Ver. Rivarin. Lomb.

Spinus -- -- -- Luccherino, o la Lecora. Itul. Lughierin. Var.

Citrinella - - Verzelino. Ilal. Zuerzelin. Vcrun. 
Friugilla Cannabina -- Montanello o Fanello marino. Itcí. Fanel grosso. Ver.

-- Linota - - - Fancllo, Faonello. Iial. Fanel piccolo, Fainel. Ver. Fanetto. Lomb.

--- domestica -- -- Passcra domestica o Capannaja. Ital. Passara, Passara grossa. Ver.

-- montana - -- -- Passara montanina. It.Passara piccola, Passara garaottina. Ver.

-- nivalis. - -- Fringuello nevale. It. Franguel de la neve. $V$ er.

Muscicapa atricapilla.

Motacilla - Luscini:- Rossignuolo, Ussignolo. It. Ver.

--- Ficedula -- -- Beccafico. It. Beccafigo. Ver.

--n nevia -- -- -- Boarola, Boarina. Benac. Bologn.

-.- Ph cenicurus -- -- Codirosso. It. Squarozolo. Ver. Cul rosso. Bologn.

-- Rubicola -- -- Pettrrosso, Pecchietto.It.Pittaro. Ver. Pettorosso. Venez.

-- Curruca - -- -- Beccafico canapino. It.

-- flava -- -. -- Cutrettola gialla o Coditremola. Iıal. Squazzacoa, Tremacoa. Ver.

-- Regulus -- -- -- Fiorrancino. It. Micheiin stellin. Benac.

--- Trochvlus -- -- Regolo comune. Ii. Tuit. Benac.

- Troglodytes - -- Sericciolo o Re di macchia o Reatino. It. Reatin, Imperatorel. Ver.

-- atricapilla - - -- Capinero. It. Ver.

-- Suecica .

Parus cristatus

- major

-- -- Cincia crestuta. It. Piansotta . Benac.

- Cingallegra, Cinciallegra maggiore; Cinciapotola. It. Parossola, $\mathrm{S}$ ecronzola, Zifotola. Yer.

- coeruleus - Parozzolino, Cinciallegra turchina. It. Pelaton. Benac.

- ater - - Cinciallegra brima. It. Paiossola piccola. Ver.

- biarmicus - - Occi boini. Ver.

- pendulinus. - Pendolini, Occi boini. Ver.

Hirundo rustica - - Fondine comune. It. Ver:

_- urbica - - Roudine domestica o il Balestruccio comune. Ital.

- Apus. - - Rondine maggiore o il Rondone. Ital. Rondone, Dardaro. $V e r$.

Caprimulgus curopisus -- Calcabotto europeo . Ital. Lattaca: vre. Benac.

Ma che vi dirò de' rarissimi insetti, delle elegantissime farfalle, che libano gli umori nettarei sui fiori delle 
benacensi pendici, o albergano nelle acque, o s'ascondono nel fimo, o si pascono dell'erbe dei prossimi monti? La loro enumerazione eccederebbe i limiti d' una lettera, ned io sarei da tanto. Abbandono quest'oggetto all' amico Bernardino Angelini, per opera del quale escirà spero fra non molto !'Entomologia veronese. Intanto per non lasciare al tutto digiuna la vostra curiosità $m$ ' accontentero d'annoverarvi alcuni per noi rinvenuti, pregevoli o per la rarità o per l'eleganza delle loro forme e colori. La nomenclatura è quella del Systema naturce di Gmelin, dove non sia

citato altro autore.

\section{COLEOTTERT}

Scaralæus sacer. Sui colli. mobilicornis. M. Baldo.

- nasicornis

- Fullo. Sui monti Lessini

- Ticentini.

--- Silenus. MI. Minldo.

-- Eremita. M. Baldo.

argenteus.

Jucanus Capreolus

--- caraboides

--- parallelipipedus.

Nitidula strignata

Curculio harbarus

--- roridus. Valle di Ronci.

Abietis. M. Baldo.

-_ Pini. M. Baldo e Lessini.

Cerambyx oculatus

-n Salicis
Cerambyx moseliatus

-- alpinus. MI. Jícelita.

- coriarius

- Faber

_-n trifasciatus

--- hatniensis .

Mycrophagus testaceus. Faldr. Lutr. Nei funghi coriacei.

Dermestes boleti. Villers. Nei funghi.

Cara!us germanus

-.- sycophanta. Al Lagn.

Elmis Maugetii. Lutr. Dirila

Linkia verrucosa, e solto lo pietre in un ruscello pressu

la Musella sui colli.

Lampyris italica

(*) Sui Coleotteri appariscono non di rado delle macchie bianchissime, cotonose, Jislunghe o irregolari, le quali coprono a modo di anello le articolazioni, specialmente del capo col tronco, e del tronco coll' addome. Osservate tali chiazz: a microscopio composto mi venae veduta un' infunità di esilissimi filamenti sempliri, nudi, ed eguali, talora affastellati, i quali spuntano da uno strato bianco. Da clie ó conchiuso essere un Bisso o Fungo bissoideo appartenente al genere $\boldsymbol{D} e$ matium del Persoon, e l'ò chiamato $=$ Dematium colcopterums: cespitosum, coufluens, album, gossypium referens. Milii. 
Elater virescens. M. Baldo .

_- carbonarius

--- sanguineus

- germanus.

Meloe bimaculata. ' ill.

Litta vesicatoria Gmel. Micloe vesicatoria Vill.

Hylesinus oleiperda. Fabr. Sugli Olivi.

Tenebrio rugosus, Vill.

Staphylinus hirtus . In Ortigara di M. Baldo e al Benaco. EMITTERT

Gryllus italicus

\section{- tataricus \\ - turritus \\ - nasutus \\ -n subulatus}

culá albá dorsali.

Mantis religiosa

_- oratoria.

Cimex eysptiacus

- albo-lineatus

- scapha. Vill.

-_ Allionii.

Blatta lapponica. Nei boschi . __ orientalis. Nelle case.

- germanica.

Fulgora europæa. Sui colli .

Cicada hæmatodes

_ - aurita. Presso il Po.

Coccus ol ex. Latr. Oliv. Sugli Olivi del Benaco

LEPIDOTTERI.

Papilio Apollo. II. Baldo e Lessini:

- Cardamines

_- Ilia

- - Iris

- Machaon

- Lathonia

- Antiopa

- Atarlanta

- ci album. Vill.

Phaliend Aesculi

- Caja

- Catax
Phalsena Paronia major

- - media

- minor

- Mellonella Alni

Napelli. M. Baldo.

- Hera

- Fagi

- Sponsa

- Nupta.

Sphinx Oenotherie

- Nerii

_- Convolvuli

- Pinastri

- Euphorbia

—_ carniolica. Sui colli .

_- ichneumoniformis

__ fuciformis. Sui colli . Atropos . NEUROTTERT .

Libellula pedemontana .

Myrmeleon libelluloides

_- formicarius. Sui colli

- Formicalynx. Al piano.

_- italicus. Vill.

—- barbarus.

Phryganca grammatica. Vill. _- reticulata. Al piano.

Hemerobius maculatus.Sui colli. IMENOT TERI.

Apis argillacea. Scop. Vill. sui colli.

-_florentina

- - violacea

-_ fulviventris

- manicata

- luctuosa

- sylvarum

- lapidaria .

Vespa unguiculata. Vill.

__ pomiformis. Esciva dal

culmo della Zea Mays.

Mutilla europrea. MI. Zevola.

Sirex Gigas. Sui monti.

pygmæa. Nei prati.

Scolia flavifrons. M. Baldo.

Leucospis gallica. Vill.

_- dorsigera. Al piano. 
Chrysis aurata

- iguita

- cyanea.

Cynips Quercus peclusculi.

Nei boschi.

\section{Cymps (Ouercus petioli \\ - Iagi}

- psenes. Al Prato della farme presso C'ampione.

Dopo tanti scritti discordanti publlicati intorno alla Caprificazione, non fia cosa al tutto vana l'addurre alcuna nuova osservazione a dilucidazione d'un tale argomento. La Caprificazione è quell'operazione usata fin da tenupi i più rimoti e ancora a di nostri in Levante, la quale à per oggetto di promuvere la maturazione dei Fichi. Consiste nel collocare sopra un albero di Fico, che non genera che Fichi secondarj, alcuni Fichi primaticci o Fichi-fiori infilati. Sortono da questi deşli insetti ( $C_{\text {ynips }}$ psenes, Linn. Diplolepis Ficus Caricce Latr. ) carichi di polvere fecondatrice, i quali s'introducono per l'occhio ne' Fichi secondarj, e fecondando i semi accelerano la maturazione del frutto. Una tale dottrina da molti seguita, infra i quali è pure il gran Linneo, vien particolarmente dal Pontedera nostro rifutata. Pensa questi, che gl'insetti arcelerino la maturita dei Fichi allo stesso modo, che vengono pii presto mature le nostre pere e mele, le quali furono ferite dagli insetti. Altri naturalisti all'opposto reputano, nè a torto siccome io giudico, essere la Caprificazione un effetto dell' abitudine degli uomini e nulla piu. Diflatti in molte contrade di Levante non si conosce la Caprificazione, nè in Italia, in Francia, in Ispagna. E l'Olivier, ch'c dimorato lungamente nelle isole dell' Arcipelago, asserisce, che da qualche tempo andavasi trascurando wna tale operazione, e ad onta di ciò otteneansi Fichi ottimi a mangiarsi. Oltracciò dove fosse mestieri alla maturazione dei Fichi, che la polvere seminale penetrasse nei Fichi feminei, sia per sé stessa diseminata nell' aria, sia per opera dei Cinipi, siccome si vuole, ogn'nom comprende, che tali Fichi maschi non potrebbero fecondare e i Fichi femine già cresciuti, e quelli che appena appariscono sull'albero, e quelli clie non sono ancora apparsi, e che tuttavolta maturano oltre due mesi appresso.

Nelle nostre contrade il Cinipe psene non depone giammai le nova nei Fichi coltivati, i quali tutti malurano i loro frutti, ad onta che diansi molie varieta, ,he generano solamente femine tauto nei frutti primaticci che nei secondarj, siccome o altrove fatto vedere. Abita egli costantemente i Fichi selvatici, i quali appo noi non maturano giammai il frutto. Io ó notato tre varietà di Fico sclvatico. La prima a frutto d'un verde sovente azzurrognolo, la seconda a frulto nero: sono vo!garissime sugli antichi edificj e nei luoghi sassosi : ambedue portano soltanto Fichi feminei. La terza genera frutti, ne' qnali avvi moltissimi lior: feminci con pochi maschi presso l'uc- 
chíetto. O'veduto quest'nltima varietà soltanto sulla sponda bresciana del Benaco particolarmente al luogo detto il prato della fame: Ai priini d'Agosto aperti i suoi frutti offerironsi zeppi di Cinipi. Ogni ovario contenea un individuo. Alcuni di essi erano tuttora nello stato di larva, altri prendevano a trasformarsi e ad escire, altri erano già nello stato d'insetto perfetto. Ad onta di ció tntti tali frutti cadevano dall'albero immatưri, come cadẹvano gli altri Fichi androgini clella stessa varietà non penetrati dai Cinipi .

Diplolepis gallie liuctoric. Encycl.

- Quercus folii. Encycl.

- bedeguaris. Encycl.

Sphex interrupta *

fi insubrica. Eatro le cạse

\section{DITTERT.}

Asilus flavus. M. Baldo:

- crabroniformis.

-

- ferox. Vill. 11 Benseco

presso Sermione.

Tipula quadrimaculata . lióverè di Velo.

- pectinicornis. Al piano. Oestrus veterinus. Bracy Clark.

Presso gli armenti in valle

Fraselle di monte Zevola.

Bonblyylius ater.

Musca conopsoides. Fill.
Musca crabroniformis. MI.Zevo-

- meridiana

_.. Hottentolta. Vill.

- coccinea. Vill.

Tạbanus al pinus. I'ill. Mr. Ballo. - albipes. Gm. M. Baldo. ATTERI.

Scolopendra Gabrielis . Nei lelamai.

Onișcus pustulatus. Sui colli - Armadillo.

Julus arborum

\section{- complanatus}

Lepisma thezeana, Fabr. Latr. Nonte Bulca.

Aranea punctoria. Vill. Nelle cantirse.

- sarruinolenta.

Phaliugium Opilio. Nelle case. - cornutum. Nei boschi.

- cancroides.

Io terminerò di favellarvi degli animali coṇ una parola intorno ai nostri serpenti. Sei sono i serpenti della provincia nostra, quattro soli dei quali però mi $\mathrm{i}_{j}$ è avvenuto vedere nei contorni del Lago. Il primo e più comune è la Biscia (Carbonazzo, Bastoniere Ver.) Coluber Natrix, animale innocente, che si moltiplica prodigiosamente vicino alle acque, e che vive principalmente di pesciolini e di rettili acquajoli-. Di questo ò vedúto una varietà nei contorni di Peschiera d'oltre un metro di lunghezza. Il suo colore era più oscuro dell' ordinario e a macchie indistinte, "má nón 
mi è avvenuto di poterla esaminare particolarmente. Un'altra varietà di gran mole abita ne' cespugli lungo i torrenti delle colline. Il suo colore è ceruleo con macchie nere ai lati, e con due serie di macchie più piccole di ugual colore per di sotto. Il secondo è la Vipera del Redi, Vipera Redi Lacépéde, Coluber Redi Gmelin: mi venne questa veduta sui colli veronesi, ma è assai rara. Il terzo è la Vipera comune Coluber Berus, la quale nei contorni del Benaco, e alle falde di monte Baldo à una tinta di un rosso più carico che altrove, massime nella coda, l'apiçe della quale è pinto in giallo. Il quarto Golubro ( Angio Ver.) abita nei luoghi asciutti e pietroși. Io l'ò rinvenuto su tutti i cólli veronesi, ai piedi di monte Sumano, e sugli Euganei, onde potrebbe chiamarsi il Colubro veneto. Non so se alcuno de' nostri naturalisti ne abbia favellato. Si può díre, che appo noi tenga il luogo del Colubro verde-giallo (Coluber viridi-flavus, Couleuvre verte et jaune Lacépéde, Milò, Melò, Milordo Lombard.), il quale in Lombardia predilige pure i luoghi aridi e sassosi, particolarmente nei contorni di Brescia, Milano, Pavia, Novara. Nella provincia nostra all' opposito il Colubro verdegilallo fu dá me veduto solo nelle basse regioni verso il Po. Ma per ciò che spetta al nostro Angio pare desso il Coluber flavescens dello Scopoli e del Gmelin. A' dugento venticinque scudi addominali, e settantacinque paja di scudetti codali. La sua ordinaria lun'ghezźa è d'un metro, ma arriva per avventura a un metro e meźzo. La sua testa è ovale, il corpo coperto di'squame liscie ellitico-esagone, superiormente fosco, per di sotto giallognolo. È d'una agiliti senza pari . 
34

Il suo cibo più ordinario sono i rospi. Fugge velocissimo all' avvicinarsi dell' uomo. Più volte ò tentato sospingerlo al morso coll'irritarlo, ma sempre indarno, ad onta che gli avverrebbe di ciò fare, potendo spalancare le fauci, ed essendo armato di denti acutissimi. L'ultimo serpente è l'Anguis fragilis ( $\mathrm{Or}$ bisolo Volg.), con tale aggiunto distinto, perchè alla minima percossa si rompe. E l'unico che abiti anche sugli alti monti, avendolo più volte rinvenuto alla metà dell'altezza di monte Baldo. E il più torpido fra i nostri serpenti, ed il più breve. E fornito' d'occhi lucidissimi, ma piccoli assai, la qual cosa fu cagione alla credenza degli abitatori di Baldo e dei contadini Lombatdi ch' e' sia cieco, e di chiamarlo con tal nome. Appo loro è pure opinione comune, che sia velenoso, tuttochè innocentissimo e impotente a mordere .

Tornati adunque a Bardolino, siccome ò detto, l'ospite nostro e compagno ne strinse a rimaner seco alcuni di, e ben potete credere se fu lieto soggiorno. Il mattin rugiadoso ne scorgeva alle vicine terre, dove sommamente ci dilettava il vedere e la coltivazione de' poderi, e gli usi degli abitanti. Si vivea la sera in lieta brigata, e godevamo sovente solcar al pallido lume della luna le tranquille onde benacersi. Se non che ne perturbavan alquanto il piacere i pescatori con que' loro inganni tesi agli incauti abitatori dell' acque. Miseri! mentre qua e là mossi da amore 4 van guizzando caggiono nella rete ministra di morte. Allorchè poi il sole era più cocente davam'opera agli studj naturali, o alla piacevole ombra d'un vicino bosco ne ricreavan l'anima i carmi de' nostri poeti. 
$\mathrm{Ma}$ in proposito di boschi non so rimanermi dal favellarvi d'un fenomeno, cui ne indussero a contemplare quelli che vestono le pendici benacensi, e il fianco di monte Baldo. Intendo io la direzione de' rami inferiori degli alberi, la quale al dire di Dodart (Mem. de l'Académie Française an. 1699. p. 73.) suol essere parallela al suolo, donde nascono, sia esso o non sia orizzontale. Il Dodart voi ben sapete, che spiega il fenomeno per mezzo de' due angoli, che formano le fibre dell'albero ( cui suppone continue, rette, e parallele dall'estremità delle radici a quella dei rami), l'uno alla base delle radici, l'altro alla base dei rami. Io non prenderò in particolare disamina cotale ipotesi, la quale non è sostenuta nè da osservazioni anatomiche, nè ảa fisiologiche. Noterò solo, che il parallelismo de' rami inferiori degli alberi annosi col suolo n'è sembrato sensibile solo nei casi, dove la inclinazione del suolo coll'orizzonte era somma : nei piani dolcemente inclinati non iscorgeasi differenza nella direzione dei rami dei due lati degli alberi. La quale osservazione e la nota disposizione dei rami a seguire la luce ne ànno indotti a pensare, che il fenomeno del parallelismo de' rami degli alberi, che si elevano dai piani sommamente inclinati, provenga dal bisogno, ch'ànno essi della luce. Imperocchè nel lato dove l'albero forma angolo acuto col suolo i rami onde godere la luce debbono elevarsi, e que' che non possono ciò eseguire periscono, come scorgesi negli alberi antichi, ne' quali il peso de' rami superiori comprimendo gl'inferiori verso il terreno osta loro d'allungarsi, laddove nel lato opposto possono i rami estendersi a loro agio. 
Una delle occupazioni nostre predilette nelle ore calde furono le osservazioni microscopiche sulle Conferve e sulle Linchie o Tremelle. Voi conoscete intorno a questo argomento le belle scoperte di Adanson, Corti, Fontana, Muller, Schérer, Orazio Saussure, Chantrans, Roth, Dilwin, Bory, Vaucher . A quest' ultimo in ispecie dee saper grado moltissimo la fisiologia vegetale, perocchè le di lui fatiche sparser fulgida luce sul vario modo di crescere e moltiplicaŕsi di cotali piante; sicchè si comprese che davasi un istesso nome a una famiglia composta di molti generi differenti. Anzi ciò ch'è più, confermate le osservazioni di Adanson, Corti, Saussure, videsi che annoveravansi fra le piante esseri pertinenti al regno animale, quali sono le Oscillarie. Ciò non pertanto regna tuttora controversia appo i naturalisti intorno alle osservazioni del Vaucher. Quelle in ispecie che risguardano le Oscillarie e le Linchie sono contrastate . E non solo negasi da taluno alle Oscillarie il moto di locomozione, ma e il notissimo di oscillazione non vuolsi ammettere come spontaneo, e s'ama meglio riferirlo al rapido accrescimento d'i cotali esseri, ad elasticità o ad altra causa meccanica. La sicura via di trarsi da tali dubbiezze è l'osservazione e lo sperimento, e noi la tentammo. Abbiam dunque raccolte tutte le Conferve, Oscillarie, e Linchie, che ne si paravano in copia nei seni e nei porti del Lago, e nei ruscelli, e nei fonti, e nei lunghi opachi, e sovr'esse abbiamo intraprese le nostre osservazioni.

Io nón entrerò in particolare racconto di tutte le osservazioni per noi istituite sulle Conferve. Dirovvi solo, che le determinate mercè 1' esame microscopico 
furono la Conferva aurea del Dilwin, la Polysperma glomerata, l'Hydrodyction pentagonum, le Conjugata princeps, C. cruciata, C. porticalis, C. angulata, C. lutescens, la Prolifera parasitica del Vaucher, e parecchie altre non descritte, delle quali ò pubblicato la descrizione nel primo fascicolo delle piante nuove o rare del veronese. La più volgare fra queste è la Conjugata princeps o Conferva jugalis del Muller. Noi ebbimo la ventura di osservarla in ogni stato, vale a dire prima di accoppiarsi, nell'atto che accoppiavasi, e dopo. Sommo diletto ne porgeva la contemplazione di questi esseri singolarissimi in tanti varj aggruppamenti. Qui filamen ti liberi, là che imprendevano a mandare il tubetto comunicatore; e dove la spirale già disponevasi a passare nella celletta corrispondente, onde costituire il globetto moltiplicatore, e altrove erano i filamenti accoppiati, e già appariva in una cavità il globetto, mentre la corrispondente era vôta. Tutte le altre Conjugate furono da noi rinvenute accoppiate e frammiste insieme alla maggiore, ad eccezione della Conjugata angolata, che ne si offerse talora isolata .

Ma le Oscillarie a sè chiamavano oltre ogn'altra l'attenzione nostra; nè fu malagevole accontentarla , perocchè ne rin:enimmo in copia nei fossi ove l'acqua è stagnante, sulle strade, nei luoghi opachi, e negli atrj delle case. Si conoscono le Oscillarie a prima giunta, perchè i loro filamenti insieme ammassati formano strati lucenti e come vellutati d'un verde carico, e talora bruno o sanguiguo. Sentono anco un cotal odore disgustoso, il quale vien tosto fetente serbandole per alcun tempo nei vasi. Il carattere perì 
essenziale di cotali esseri singolari, onde furono chiamati Oscillarie, è il movimento incessante di oscillazione da destra a sinistra o da sinistra a destra of sicchè vanno mutando continuamente luogo . Corti paragona tale movimento a quello de' serpi . Cinque furono le Oscillarie, che pei loro caratteri particolari si fecero distinguere. Però non sono riescito a riferirle sicuramente alle già descritte dagli autori. Laonde prenclerò a esporre brevemente i loro caratteri, e in qual modo appalesavano i loro movimenti .

I filamenti o tubetti della prima specie possono valutarsi d'un 300 di linea in grossezza. Sono trasparenti, ed ànno un'estremità più rotonda dell'altra, la quale è come troncata. Constano di anelletti, che sono il doppio più larghi che lunghi. La figura del Vaucher cui più s'assomigliano è quella della Oscillatoria fusca tav. I5. fig.9. Abita questa Oscillaria nel fondo dei fossi d'acqua stagnante, formando arcpj verdissimi tappeti. Cresce anche nella vasca della fonte dell'Orto botanico di Verona mista alla' seguente. Essa ne à palesato chiarissimamente il movimento di oscillazione e di locomozione. Talora il movimento di locomozione ne si offerse non angolare, ma retto, eguale, e continuato, sicchè vedeansi i filamenti attraversare in linea retta il campo del microscopio.

La seconda è intorno un terzo più piccola della prima. Rinviensi negli stessi luoghi. I filamenti sono trasparenti, e i loro anelli visibilissimi, e all'incirca tanto larghi che lunghi. Formano tappeti d'un verde cupo, e si dispongono nello strato o letto regolarmente a stella, con una estremità allo infuori. I suoi movimenti laterali sono lentissimi, nè siamo pervenuti a 
scorgere il moto di progressione. La figura del Vaucher più alla nostra somigliante ì quella della Oscillatoria Adansonii tav. 15. fig. 6.

La terza consta di tubetti la metà più piccoli di quelli della prima. Sono essi trasparenti, e senza visibili anelli. Formano sul suolo e sulle pareti delle case volte a bacio delle chiazze di color verde volgente al rosso scuro, e talora simili al sangue corrotto. $L^{\prime}$ ò rinvenuta più volte anche in Verona negli atrj delle case, e particolarmente in quello del palazzo Bevilacqua, altre volte mia abitazione. Inclinerei a crederla l'Oscillatoria lavigata tav. 15. fig. 10. del Vaucher. I movimenti nei lati e locomotivi appajono distintissimi, ed è la specie che più li offre simili a quelli de' serpenti, siccome li descrive il Corti. In questa siccome di più facile ritrovamento ò istituito moltissime osservazioni, e mi sono accertato del modo, onde si moltiplicano cotali esseri, vale a dire per separazione de'filamenti in più parti, siccome ne àn rivelato il Corti ed altri. È tenacissima della vita . Sovente l'ò raccolta sul suolo dopo molti giorni, che non era caduta pioggia, e però ristretta in membrana aridissima e fragile. $0^{\prime}$ fatto pure seccare delle ciocche per più giorni al sole cocente. E immerse in seguito nell' acqua, appena lo strato erasi rammollito, e tosto i filamenti si disponevano a stella, e prendevano a muoversi .

La quarta di tutte la più piccola parmi certo l'Oscillaria alba tav. I5. fig. Ix. del Vascher. I suoi filamenti sono trasparentissimi e senza visibili anelli. Essa mi è comparsa tre volte sulle Conferve acquajole serbate per qualche giorno ne' vasi onde studiarle. 


\section{0}

Forma sovr'esse un velo bianco a luogo a luogo più fitto. I suoi movimenti laterali sono lentissimi, nè m'è avvenuto vedere quelli di locomozione .

La quinta finalmente sembrami l'Oscillatoria parietina del Vaucher tav. 15 . fig. 8. E della grandezza della prima con anelli distintissimi, larghi la metà più che lunghi. Ambedue le estremità sono rotonde, non però terminate da globetto, come asserisce il Vaucher della sua. Forma ampie macchie d'un verde cupo sulle pareti dei condotti d'acqua, e l'ò rinvenuta inoltre sulle ruote dei molini appo Verona, massime in quelli lungo la via d'Avesa. I suoi movimenti oscillatorj e locomotivi si manifestarono chiarissimamente.

Dopo aver esaminato le nostre Oscillarie al microscopio composto, ed esserci assicurati della spontaneità de' loro movimenti, abbiam voluto sottoporne alcune all' azione del microscopio solare. Nè vi so dire quanto fosse il nostro diletto nel vedere cotali esseri minutissimi muoversi come tanti serpentelli per diversi lati, quale con maggiore quale con minore lentezza $\left(^{\star}\right)$. Appresso piacquene cimentare le Oscillarie in varie foggie, esponendole all'azione del caldo, del freddo, e dei varj liquori. Però non ne avvenne scorgere la differenza di celerità ne'movimenti notata dal Corti . Uguale era la lentezza tanto nell'acqua a pochi gradi sopra zero, come nella riscaldata oltre trenta. Lo stesso occorrea quando si tuffavano in varj liquori come nell' aceto, nell'alcool, nell'orina : solo dopo al-

(*) Questa osservazione fu ripetuta parecchie volte collo stesso lieto successo nella scuola di fisica di Verona alla presenza del mio illustre amico Prof. Giuseppe Zamboni, e di tutti gli allievi. 
quanti momenti cessava ogni movimento, indizio della virtù ledente di cotali liqguori. Il fenomeno per noi rinvenuto vero è la sensibilità delle Oscillarie all'azione della luce. Allogavansi delle ciocchette di Oscillarie in un recipiente ricoperto di carta nera alla quale faceasi da un lato un forellino, onde penetrasse la luce, e vedeansi indi a poche ore tutti i filamenti sparsi per l'acqua del recipiente ammassati verso il forellino. Rivolgevasi allora il vaso in modo, che il pertugio rimanesse dal lato opposto, e poco tempo dopo scorgevansi le $\mathrm{O}$ scillarie affullate verso il lato illuminato. Da tutte queste osservazioni pertanto, ma particolarmente dal movimento di locomozione noi abbiam conchiuso essere le Oscillarie da ascrivere veramente al regno animale.

L'analogia che regna infra le Oscillarie e le Linchie ne indusse a considerare queste pure particolarmente, e a conoscere le loro abitudini. Constano le Linchie, com'è noto, d'una membranella piena d'una sostanza gelatinosa, nella quale nuotano molti sottili filamenti moniliformi. Cotale membrana a certa età si fende, e i flamenti racchiusi si separano in tanti globetti, ciascuno dei quali moltiplica la specie. Dalle quali cose si trae, che la maggior differenza, che avvi tra le Oscillaric e le Linchie è la membrana, che serve loro d'inviluppo, perocchè la gelatina delle Linchie viene rappresentata nelle Oscillarie della materia ontuosa, che loro serve di letto. Due Linchie noi abbiam preso a considerare primamente in questi luoghi, la comune o Nostoc ( Tremella Nostoc Limn.), che a noi occorrea in copia ne'viali del giardino e sul vicino pratello in riva al Lago, ove escivarno a 
diporto, e la Linchia verrucosa ( Tremella verrucos $a$ Linn.) rinvenuta appiccata ai sassi del fondo d'un ruscello. Distinguesi questa dalla Nostoc perchè più soda, non pieghettata, ma tondeggiante e bernoccoluta, e inoltre è sempre applicata ai sassi o alle zolle e non mai libera. I filamenti poi della verrucosa non sono si chiaramente moniliformi come quelli della comune. A noi caleva più che altro conoscere $\mathrm{i}$ movimenti, onde al dire del Vaucher sono forniti i filamenti. Due ne osservò egli nelle Linchie: uno consisteva in una repentina contrazione sui lati, ed era rarissimo, e avvenía solo quando agitavasi l'acqua; l'altro di locomozione ma lentissimo, sì ch'era bisogno onde avvedersene osservare attentissimamente la posizione de' flamenti infra loro. Aggiunge poi, che il momento più propizio onde scorgere tal movimento è quando la membrana sta per ischiudersi, e la gelatina è più liquida .

Abbiamo tolto a osservare primamente la Linchia comune, ma non siamo riusciti a vedere alcun movimento ad onta d'ogni mezzo posto in opera, e agitazione, e liquori acri, e il calore. Più avventurati fummo nell'esarne della Linchia verrucosa, che abbiam rinvenuta nelle sue diverse età . Abbiam veduto più e più volte separarsi sotto i nostri occhi i filamenti in globetti, pria l'ultimo, e a mano a mano gli altri; talora però disfeasi a un tratto tutto il filamento in globetti, massime quando l'individuo era adulto perfetto. Ma onde avvederci de' loro movimenti dopo molti inutili tentativi fummo astretti a ricorrere all' artificio, suggerito dal Vaucher, di disegnare sopra un pezzo di carta la disposizione rispettiva dei filamenti 
nella propria gelatina, e osservarla ivi a qualche tempo. Abbiam ripetuto gli sperimenti per ben dodici volte, valendoci delle Linchie allo stato di maturazione, e da essi ne parve di poter asserire, che i filamenti della Linchia sono dotati di un movimento, benchè sovrammodo lento, di locomozione; conciossiachè il più delle volte abbiam rinvenuto la loro posizione assai cambiata, quali essendosi avvicinati, quali allontanati .

Ma voi certamente sarete compreso dalla meraviglia all'udire ascrivere alle Linchie una sostanza, che dal pien consenso de' Botanici è annoverata fra' Licheni . E questo il Collema pulposum dell'Acharius (Lichenogr. p. 632.) o Lichen pulposus del Bernhardi, comunissimo sui sassi di monte Baldo, sulle sponde del Benaco, e su tutti i colli del Veronese e delle vicine provincie. Varia egli senza modo, ora vestendo una forma più propria di Linchia, crespa o lobata, il più senza vestigia di apotecj, ed è il Lichen crispus di Linneo, ora venendo come granuloso, ora coprendosi nel centro di apotecj di color rossiccio, e ammucchiati, qualità proprie del Collema polposo. Alcune di tali varietà ò a voi più d' una volta spedite. Contuttociò noi abbiam in esse riconosciuti i caratteri delle Linchie. Imperocchè non solo nella sostanza polposa costituente il corpo o tallo, e negli apotecj o organi che mentiscono la fruttificazione, abbiamo scoperto un'infinità di esilissimi filamenti moniliformi, che si separavano in globetti sotto i nostri occhi; ma ciò ch'è più, avendo posto in opera l'artificio di che vi ò detto favellando della Linchia verrucosa, ci siam più d'una fiata avveduti d'un cambiamento di luogo de' filamenti fra loro. 


\section{4}

Una pianta singolare della famiglia delle Alghe ab. biam discoperta nell' acque placide dei seni massime nell'Isola dei Frati e a Sermione. Mi soccorre di averla anco rinvenuta, or volge il quarto anno, nei fossi delle risaje di Villimpenta presso il forte d'Attila. Sta essa appiccata per la parte inferiore alle foglie della Vallisneria, delle Carici, dei Scirpi e d'altre erbe palustri; e dove ne venga staccata galleggia alla sopraffaccia dell'acqua. La sua consistenza è gelatinosa, e si spappola di leggieri fra le dita; il colore giallognolo. E tubulosa e tortuosamente ravvolta quasi a modo di budello. La sua figura s'avvicina alla conica, da acuta e sottile all'estremità inferiore a mano a mano dilatandosi verso l'apice, ch'è assai ottuso o ben anco globoso. Osservato un brano di essa a microscopio presenta due membrane esilissime e trasparenti senza visibile organizzazione, le quali racchiudono una gelatina, entro cui nuota un' infinità di filamenti. Cotali filamenti sono alquanto più lunghi di quelli della Linchia Nostoc, ma non moniliformi. Sono più grossi a un'estremità, e conservato nell' acqua per alquanto tempo qualche pezzo staccato, e osservato a microscopio ò veduto la più parte de' filamenti disporsi a stella nei contorni dei pezzi, coll'estremità più grossa rivolta internamente e coll' apice sporgente fuori della gelatina. I filamenti sparsi nel centro dei pezzi aveano varie direzioni, e alcuni appariano anche incurvati. Una tale disposizione simile a quella delle Oscillarie mi à fatto sospettare della loro natura animale, ma non sono riescito a scorgere verun movimento. Solo mi è avvenuto vedere la separazione della estremità più grossa dei filamenti sotto 



\section{A L L O S T E S S O.}

Lettera II.da sul monte Baldo

Dopo alcuni giorni adunque di dimora a Bardolino, come vi dicea, c'incaminammo a monte Baldo, e fummo pria di tutto a Caprino, villaggio fabbricato alle di lui falde meridionali. E posto monte Baldo alle fauci dell'alpi, che l'Italia dividono daila Germania, a venti miglia a ponente di Verona. L'Adige ne hagna le radici a levante, il Benaco a occidente, a settentrione à l'Alpi Retiche, cui si congiunge per la valle Lagarina, e declina dolcemente a mezzodì sino a S. Vigilio e Caprino. Stendesi l'alt o suo dorso da settentrione verso austro, e s'estolle sino all'altezza di 2228 metri, ed à di lunghezza trenta miglia, di larghezza undici. Il suo fianco orientale è erto e inaccessibile, quello d'occidente parte è selvoso, parte si stende in ampie valli e dolci pendici. Le più umili cime sono tutte erbose, le altissime sono ignude balze ed aspri dirupi. Ma che veduta da que' luoghi! Scorgiamo a settentrione scender dall'Alpi del Tirolo precipitoso l'Adige. Roveredo ne si para in distanza; la sottoposta valle amenissima è cospersa di borghi e villaggi; le Alpi Retiche col vertice mai sempre nevoso disposte ad anfiteatro chiudono la prospettiva. Deliziose colline appariscono a levante, che soavemente declinano in fertili valli, e l'Adige frettoloso, che ser- 
peggiando penetra in Verona, che fa di sè bella mostra; e quando sia cielo serenissimo spingesi la veduta ai monti Berici, agli Euganei, al mare. A mezzo giorno eccoci la vasta pianura dall' Alpi all' Appennino, e le Città si discoprono, e i borghi, e scorrer vedesi il Mincio e il Po. Ma tutto vince la veduta di ponente, poichè tutto vince la bellezza del Lago. Vedesi dal piè del monte, che n'è bagnato, estendersi il mobil piano dell'acque cerulee; e le rupi di fronte, e i verdi colli delle sponde, e $\mathrm{i}$ giardini, e $\mathrm{i}$ villaggi, e le isolette, e le navi scorrenti sull'ondeggiante superficie son tale incanto, la cui dolcezza non si puù con parole esprimere. Venite, amico, venite sul giogo di Baldo. Meraviglioso soprammodo è lo spettacolo, che qui offre la natura alla vostr'anima sensibile!

Nè men caro vi riescirà monte Baldo per sè stesso . Imperocchè e vi rallegreranno da un lato amplissime praterie, tutte di variopinti fiori seminate, onde olezzo soavissimo si diffonde all'intorno, e v'empieranno dall' altro l' anima di sublimità le folte selve maestose, venerabili per Faggi antichi, per Larici, per Pini e per Abeti altissimi; e qui son pendici amene cosparse di capanne, e veggonsi gli armenti pascolar tranquilli, mentre il pastore all' ombra fa eccheggiar le balze della sua zampogna; e là asprissimi dirupi appariscono, e roccie precipitevoli, e valli squallide prom fondissime, ove la natura regna solitaria e minacciosa. Nè mancan torrenti, che precipitano dalle rupi, ruscelli di dolci acque, che van rigando con lento corso il seno di amene vallette, e fonti gelidissimi circondati da salvatici alberi. Nulla poi è a dire della serenità del cielo e dell'aria saluberrima che vi si 
spira; perchè niente avanza il bello azzurro di quello, niente la leggerezza, l'elasticità di questa, sic chè par ne infonda altra vita.

E da tutte queste cose beveva io appunto una dolcezza infuita, seduto sull' erbosa vetta di Baldo, che soavemente oliva; quando piacque alla mia ventura offrirmi uno spettacolo spaventoso e sublime. Limpido era il cielo sull'alto gingo, taceva ogni vento, ed ecco sui fianchi del monte adunarsi nembi, mugghia orribilmente il tuono, guizza la folgore sotto a' miei piedi, il lago è coperto di caligine, e s'eleva in monti e si profonda in voragini. Odo intanto da altro lato il fragore e lo scroscio della grandine, e il rovinío de' torrenti, che fan fuggire armenti e pastori. La tempesta infuriò alcun tempo, ma de'venti lottatori vince quel di ponente, e disperso è ogni nembo, e la valle e di nunvo illuminata, e l' onde del lago tingonsi novellamente di cilestro; tutto è calma, è riso. E il sole che da ponente lanciava i suoi raggi uno spettacolo meroviglioso mi offerse: un arco bellissimo, non già qual sogliamo comunemente rimirarlo, ma sotto forma di settemplice circolo apparve in cielo.

Ma venendo ora a ciò che risgnarda particolarmente il naturalista, e intendendo io dirvi anzi tutto alcuna cosa intorno alla costruzione di monte Baldo, non posso non comprendere nella disamina anco gli altri monti Veronesi, ciò richiedendo l' analogia di questi con quello. I monti Veronesi sono diramazioni dell' Alpi Retiche primitive poste nel centro del Tirolo, le quali a rnano a mano declinando s' appianano negli ame- 
nissimi colli vestiti d' olivi, di viti, e d'alberi fruttiferi, alle cui falde è fabbricata Verona $\left({ }^{*}\right)$.

${ }^{*}$ ) L' estensione dclla provincia Veronese dal nord al sud, ossia da monte Pertica ad Ostiglia sul Po è di miglia nuove di mille metri ciascuno, ossia chilometri numero settanta, corrispondenti a incirca quarantadue miglia geograficlse italiane: l'estensione dall'ovest all' est cioc̀ da Sermione a Montebello ¿̀ di chilometri sessanta, o miglia geografiche incirca trentaquattro. La superficie quadrata è di tornature trecento quarantacincque, o incirca mille ottocento cinquanta miglia geografiche. Quasi la metà di questa provincia a settentrione è montuosa: segue poscia l'alta pianura, la quale a mano a mano abbassandosi termina nclle paludi verso il Po. Varj fiumi poi scorrono per essa, l'Adige, il Mincio, e i fiumicelli Tartaro, Molinella, Menago, Bussè, Tregnone, Fibbio, oltre i torrenti Fumane, Lavagno, Tromegna, Illasi, Alpone', Chiampo ed altri minori. Verona capitale della provincia giace alla distanza d'incirca sessanta miglia dall'Adriatico, al $28 .^{\circ} 40^{\prime} 39^{\prime \prime}$ di longitudine, e al $450^{\circ}{ }_{2} 6$. $^{\prime} .^{\prime \prime}$ di latitudine. I confini da me stabiliti della provincia nostra non sono quelli che à di presente, o che ebbe nelle epoche passate, i quali ora si restrinsero ora si ampliarono col variare de' governi. Io è amato prendere dei punti fissi che mi parvero i limiti naturali, e li ò seguiti anche nella sposizione della Flora veronese, che omai è giunta a suo termine.

Io qui aggiungerò le distanze verticali dalla superficic dell'Adriatico tanto di Verona, che di molti altri luoghi massime montuosi della provincia, e di alcuui delle prossime provincie. Cotali distanze furono rilevate dalle osservazioni barometriche calcolate secondó il metodo di Biot. Molte di queste le ò istituite insieme al valente mineralogo, mio dolcissimo amico e collega ne' viaggi montaneschi il Conte Ignazio Bevilacqua Lazise. Noi abbiamo stabilito col celebre Oriani l'altezza media del barometro al livello dell' Adriatico pollici

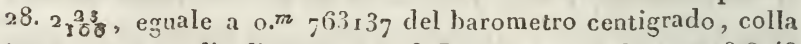
temperatura media di + io $\frac{74}{100}$ di Reaumur, eguale a $+13 .^{\circ} 43$ del termometro centigrado. E abbiamo preso per punto fisso in Verona la sommitâ dell'idrometro costrutto in pietra sull'Adige allo sboceo del rastrello di ferro presso la inaddietro Chiesa di S. Salvar Corle-regia, al punto ove sono segnati i piedi verouesi 1 4. Dalle osservazioni eseguite pel corso di 26 anni in Verona per ordine dell' Accademia d'Agricoltura, Com-

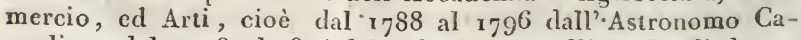
gnoli, e dal $179^{S}$ al i 814 dall' $\Lambda$ b. Tommaselli risulta l'altezza i edia del barometro riportata alla sommità dell'idrometro 
Spaziose valli scavate dai torrenti tagliano da settentrione a mezzodi questi monti, delle quali le prin-

stesso pollici $27.11, \frac{40}{10}$ nguale a $0, m 756752$ del barometro centigrado, e la temperatura media $a+10 .^{\circ} 7,668 \mathrm{di}$ Reaumur, eguale a $+13.4^{\circ} 3$ del centigrado. E però la distanza di detta sommità dall' Adriatico è di metri - 70.500 Pelo dell'acqua ordinaria dell'Adige in Verona sta-

bilito a piedi quattro dell'idrometro stesso, cioè metri 3. 429 sotto la di lui sommità Ospedaletto in valle P'ulicella. Casa Butturini a piano

terreno - - - - - - -130.49

Dolcé. Osteria delle due Torri, piano terreno - 127. 23

Lirentino. Osteria, piano terreno - - - 209.30

Ario. Osteria in faccia alla Chiesa parrocchiale,

piano terreno : - - - - - 15r. 33

Mori. Osteria in piazza, piano terreno - - - 202. 49

Garda. Livcllo dell' acqua del Lago - - 77.82

Pesena. Casa Fontana, piano terreno - - 295 . 5I

Caprino. Usteria - - - - - 275.05

Monte Baldo. Santuario della Corona, limitare della

Chiesa - - - - - - -

776. 50

Monic Croce sopra la Corona, al luogo pii eminente ove appariscono materie vulcaniche - - - - roo5. 95

- - La Ferrara. Osteria dei Dossi sopra la Chiesa - - - - - 909. oo Valle Ortigara. Alla Chicsa $\quad-1439.62$

- - Fontana di Navole, una delle pì̀ elevate di monte Baldo - - - 1675.56 Vetta di Costa bella, che confina alla valle delle Buse - - - -2044.87 Cima più meridionale di Monte maggiore, confinante alla valle delle pietre, cui credo quella che lo Sternberg chiama Costa bella - - - 2068. 80 La stessa, secondo Sternberg - -2062 . 81 Monte maggiore. Vetta detta Colma di Sascaga, la piì elevata di monte Baldo - - Secondo il Manuel topographique Militaire - - 2129 .

Secondo Sternberg - 2228. 40

_- _ Cima di valle Fincstra, secondo Sternberg
Campione, all Osteria $--1350 .{ }^{76}$ 
52

cipali sono le valli dell'Adige e Lagarina, che dipartono monte Baldo dagli altri monti Veronesi, e lungo

Honte Baldo.Acque-negrc, al luogo ove sono i testacei presi ncl tufo vulcanico - $-\mathrm{r} 375$. 32 -_ - Piano della Cenerc, all'Osteria - - Iooo. oo

- - - Miniere di Terra verde, presso la valle de'Suppiadori - - - - 1218. 52

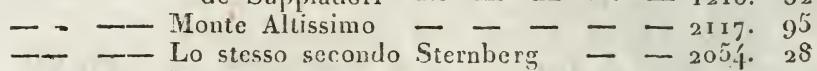

- - Monte Campo dello stesso Altissimo, ai più elevati indizj di sostanze vulcaniche - - - - - ${ }^{5} 59$ r. og

- Brentonico. Casa Ballisti - - - 689. 72

- - Casiion di Mori. Casa Banedetti - - 546. 63 -_ - Tierno, luogo delle Mesotipi e del Li$\begin{array}{r}\text { tantrace } \\ \text { Grezzana, all'Osteria - }- \pm-D= \pm 320.9^{2} \\ \hline\end{array}$

Monti Lessini. Chiesa nuova, limite della porta della Chiesa - - - - 1068. 82 Scandole - - - - 1120. $7^{5}$ Montagna dei Pezzi sopra le Scandole, ove appariscono materie vulcaniche - 1380.48 Monte Tomba, sommità, incirca - 1868.15 Roncà, all' Osteria - - - - - - 56 . 24 Monte Calvarina, alla valle di Roncà. Sommità del S. Gio. Ilarione, alla valle di Roncà. Casa Balzi 699. оо Salvioni, mezzo miglio a meriggio della villa, nella sala terrena - - -

-_ Prismi basaltini alle case Panaroti - - - - - - 258.18

- - Case Scarmana sulla via di Vestena - 386. 40 Bolca. Villaggio all' Osteria Camponogara _ - 827. 30 - Cava degli Ittioliti detta la Pesciaja Maffei - 597. 12 - Suo vertice detto la Purga - - - $-94^{6} 4^{2}$ Schio. Limitare dell' Osteria Gaole presso la piazza - 197. 19 Monte Sumano. Punta dell'Idolo - - - - i2S8. I8 - - La stessa secondo Sternberg - - 1278. 89 - - La stessa secondo Marzari-Pencati - $1250.9^{5}$ - - La stessa secondo Chiminello (Journal dc physique XIII pag. 45̃ 7$)-1208.73$ - Pra de' Pradazzi tra S. Orzo e Piovene secondo Zanolli - - - 159.17

- - Punta della cima dei Pra de' Frati, secondo Marzari - - - - 1218. $5 \$$ 
le quali scorre la via di Germania e scende il fiume; le valIi Pulicella e Pantena, l'una a destra l'altra a sinistra della cittì, e la valle d' Illasi quindi, e la famosa di Roncà di tutte la più orientale. Si congiungono essi da oriente co'monti Vicentini, a occidente il Lago di Garda li separa dai monti Bresciani. Negli antichi tempi era l'alveo dell'Adige differente da quello che scorgesi a dì nostri, e assai più alto: rnolsi anche che la valle Lagarina fosse allagata dalle

Monte Sumano. Casa Borghero, allc falde del monte, secondo lo stesso - - - 505. 09 Caselle di Roagua, le piì alte case del monte, lo stesso - - - $58.7^{2}$ Pra minore, secondo lo stesso - -878.73 Prima punta dei Pra Gerini - - 1086. 44 Cima della valle della Crosetta - - ir5i. 62 Al Conveuto - - - - - 1186. 35 Capitello deila Costa di Marco - - iris. 67 Casara dei Zanini -- - - - 1097. 54 Pozza di Mar di Faggia - - 1012.67 Capritello di merzo - - - 97 t. 40 Punta in cima del Costone di Piovene, sotto i Pra di Bovolco - - 702. 5' Al piede della costa di Priazza - 521. 34

S. Orzo . Casa Marzari, secondo lo siesso - - 3jr. 24 'Tiene. Principio del paese verso Vicenza, secondo lo stesso - - - - - 277 . II Osteria nuova, tra Vicenza e Tiene, secondo lo stesso - $21 \$ .39$ Vicenza. Base della Torre maggiore in piazza, secondo Herrissch - - - - 160. 00 Monte Novegno presso Schio, secondo lo stesso - 2006 . Monte Toro, seconclo lo stesso - - - 2122. Monte PortuIe, nei sette Comuni, secondo lo stesso - 2060. Monte Verena, nei sette Comuni, secondo lo stesso - 1820. Monte Mandriolo vei sette Comuni, secondo lo stesso 1960. Monte Toraro, secondo lo stesso - - - - 1820. Monte Coste basse nel Vicentino - - - 2060. Moute Pasubio nel Vicentino - - - - 1760. Monte Crace nel confine del Tirolo - - - 250. Monte Venda il più elevato dei colli Euganci, secoudo Strangc - - - - 
sue acque. Discorrea esso fra le falde di monte Baldo e monte Bibalo, che era continuazione del monte Pastello, e sboccava per la valle di Caprino. I Romani furono quelli, che spaccate le rupi della Chiusa dischiusero la strada di Germania e un nuovo letto al fiume. Del che indubitata prova ne porge la direzione della vetta dei monti Bibalo e Pastello, e più 1a giacitura degli strati dei monti della Chiusa, che tagliati quasi a piombo costituiscono le due sponde dell' Adige, e che si osserrano dovunque corrispondenti .

Il più elevato di questi monti è il Baldo, seguono poscia il Pertica di poco minore o forse di eguale altezza, quindi Campobruno, Tomba, Zevola, Gramulone, Alba e Bolca, i quali tutti costituiscono a settentrione la catena di confine della nostra provincia col Tirolo. Le loro vette son per quasi due terzi dell'anno coperte di neve, ed offrono croste scarme e frastagliate, punte acute, rupi orride ed ignude, caratteri che distinguono questa fatta di rocce. Qualche macchia di Mughi (Pinus Pumilio $W$.) e d'alcun altro nano arboscello scorgesi a stento sui loro fianchi. Di pascoli e di prati feraci sono coperti la più parte de' monti meno elerati, e in ispecie i Lessini. Le maestose selve di Larici, di Pini, di Abeti, e di Faggi, che un tempo li vestivano, furono a questi ultimi secoli quasi al tutto divelte; sorgente fatale dell' incostanza del nostro clima, dello straripamento de' fiumi, e d'altri danni gravissimi!

La massa che compone questi monti non meno che la rimanente catena, che circoscrive la pianura Veneta a settentrione, consta di calcare secondaria in istrati 
più o meno orizzontali, di colore il piì grigio sudicio, o perlino, o bianchiccio, o talora d'un rosso languido, di frattura affatto terrea, imperfettamente concoide, opaca e senza lucentezza. Gli strati calcari racchiudono filetti e vene spatiche, frequenti straterelli di creta, di marna, di argilla, di focaja, di silice, di quarzo, e sono zeppi d'infinite maniere di corpi organici vegetabili e animali specialmente marini petrificati, ora confusi e ammonticchiati, ora disposti quasi in famiglie, come rami, foglie, frutti di diverse piante (a Grezzana, a Bolca), serpenti, chiocciole, turbini, bucini, ostriche di maravigliosa grandezza (Roncà, Brentonico, Bolca), madrepore, astroiti, spine d'echini, raggi di stelle marine, nummali o discoliti di moltissime specic, noccioli di anomic, e particolarmente di corni d'ammone di numero e varietà grandi sopra ogni credere. Però $\mathrm{i}$ petrefatti che vogliono particolare ricordanza sono gl'innumerabili ittioliti di monte Bolca. All'altezza di seicento metri dal mare entro strati irregolarmente inclinati di schisto marno-bituminoso, alternanti colla calcare amorfa rinvengonsi tronchi di varj alberi, felci, od altre erbe di diversi climi massime marine improntate sullo schisto, e penne d'augelli, e scheletri di crostacei, di serpenti, d'insetti, ma particolarmente di pesci. I più di cotali pesci giacciono in una posizione tranquilla colle loro parti più delicate ossee e cartilaginose al tutto conservate e penetrate da cristallizzazioni calcaree o anche piritose. Le disamine intraprese dai naturalisti ànno rivelato appartenere altri ad esseri tuttora viventi nell' acque salse e nelle dolci dei climi nostri e dei lontani, ed altri molti essew per anco 


\section{6}

sconosciuti. Oltre quelli già descritti ed incisi nell'insigne Ittiolitologia Veronese avvene moltissimi recentemente scoperti, che adornano i gabinetti dei Signori Gazzola, Castellini e d'altri naturalisti, e il loro numero va tuttodi crescendo.

Ágli ittioliti voglionsi aggiungere le osteoliti scoperte nei monti di Valmenara presso Grezzana, e quelle rinvenute nella breccia calcare di una grotta al Cerè presso Alfaedo, e sovrattutto le smisurate zane e le ossa di elefanti disotterrate al Serbaro presso Romagnano, con quelle di cervi e d'altri quadrupedi dell' ordine delle fiere e de' ruminanti, coperte da un terriccio untuoso, e penetrate da uno spato calcare .

Ma tornando alla roccia calcare dico, che avvi diversi luoghi dei monti nostri, ne' quali essa acquista un granito più fino e più duro, riceve palimento, e costituisce i pregevoli marmi, de' quali è ricca la provincia veronese. Vi ò già fatto cenno dei leggiadri marmi di Torri. Bellissimi pur sono il giallo-rosso fosforeggiante di Brentonico, il rosso venato di S.Ambrogio, il marmo lumachella di S. Vitale, ch'è a fondo rosso variegato dalle osteoliti bianche, e la lumachella opalizzante fosforica del monte Pernise presso Lugo $\left({ }^{*}\right)$. Però a mano a mano che ci eleviamo e c'interniamo ne' monti la calcare si dispone in istrati meno regolari e diversàmente inclinati. Scompariscono gli strati di silice e di focaja, e più rare sono le petrificazioni. Molte cime di monte Baldo come Costabella,

${ }^{\star}$ ) Si annoverano oltre jo specie di marmi veronesi, de? quali però la metà si rinvengono soltanto erratici. 
valle Finestra, bocca di Narene, Altissino, sono formate di calcare ora squamosa e lucente, ora a grano finissimo che passa in oolite a globetti più o meno minuti, più o meno pronunciati. Fra le varietà di calce carbonata de' nostri monti due meritano particolare menzione : una è la Calcare iu bastoni affistellati, o Calcare bastonite comunemente detta madreporite. Noi ne abliamo rinvenuto un gran masso erratico a manca della strada che dal Campedello scorge al Cambrigaro presso la Ferrara di monte Baldo, staccato forse dall'alta pendice, e colà precipita dal vicino torrente; l'altrà è quella bella varietà di bastonite non rara in monte Viale e in altri luoghi del Vicentino, la quale chiamano tartuffite perchè percossa col martello manda odore di tartuffo. Trovasi questa presso Castagnì.

Le spelonche che soglionsi aprire ne'monti calcari, non mancano a noi pure, come al Ponte di Veja, al Covolo di Velo, al Pralungo sotto l' Ortigara in monte Baldo, e altrove. Niuna però merita particolare menzione, laddove non vogliansi eccettuare quelle del Corolo di Velo, ove è fama, che siensi rinvenute delle ossa di orso. Plammenteremo piuttosto una curiosità singolarissima, che offresi nei monti oltre Lugo, detta il Ponte di Veja. All'ingresso d'una valle tutta circondata di dirupi calcari, che riempiono l'anima d'un dolce orrore, prolungasi un arro gigantesco d'una regolarità ed armonia meravigliosa. Consta esso di strati di marmo variopinto. La sua grossezza è di metri 6, 84, la larghezza di 17, 10, sicchè porge comodissimo varco dai due lati della valle. Però le due facciate non sono egualmente estese. Due scogli alti 
metri 29, 4I sostengono a modo di pilastro l'arco orientale, che s'apre con una corda di 39 metri sopra 2I, 20 di saetta; nel mezzo avvi poi un frontalo di metri 3, 42 di prominenza. La corda dell' arco occidentale, il quale è assai irregolare, ascende a metri 52,66 . Due spelonche dischiudonsi ne' suoi fianchi, una delle quali più ampia s'addentra per lango tratto nel monte. Un ameno ruscello, che lentamente mormorando discorre sotto l'arco precipita a pochi passi dall'altezza d'oltre settanta metri, e il bordo della cascaba sporge in un secondo arco di metri $\mathrm{r}_{9}, 82$ di corda sostenuto egualmente da due alti scogli, ove è scavata altra grotta meno estesa.

Rispetto alla roccia primitiva, che serve di base ai monti Veronesi, essa non si discopre in veruna parte, ed è mestieri dedurla dall'esame de'continui monti Vicentini, Bresciani, e Tirolesi. Le rocce che più comunemente si mostrano alla base sono lo schisto micaceo e l'argilloso, ai quali sovente è sovrapposta nn'arenaria macigno, o una grauvachia costituita da un impasto di ciottoli di quarzo grossolani o minutissimi, ove predomina talora quasi al tutto il cemento argilloso misto a osilissime squamette di mica, costituendo di tal guisa l'argilla schistosa.

L'unica roccia, che oltre la calcare meriti considerazione sui monti Veronesi è quella intorno la quale pende tuttora sonina controversia appo i naturalisti, massime fra quelli che non ànno viaggiato in Italia, se sia di origine acquea o del fuoco. Il trappo (trappo stratiforme o secondario de' Werneriani) modificato in basalto, in vachia, in mandoloide, in tufo vulcanico e basaltino, o direm noi diverse fatte di lave 
veggonsi apparire fra la calcare in moltissimi luoghi . Le falde settentrionali di monte Baldo constano in molta parte di tufo vulcanico e di vachia, e i villaggi Castione, Besagno, Tierno, Brentonico sono piantati sovr'essi. Torna poscia ad apparire ora tufo vulcanico o basaltino, ora pozzolana, ora vachia, ora basalto amorfo a S. Valentino, a Pozza ferrera, ai Pianetti, in Campo sull'Altissimo, alle Scalette, ove continua lungo i fianchi deI torrente Aviana, sui quali sono le cave di talco zografico. Il tufo vulcanico e la vachia sbuccan fuori di nuovo sulle coste dell' Artillon e deli' Acque negre, alla Ferrara, alla Croce presso la Corona, e molti pezzi erratici di basalto amorfo ò rinvenuto al Cerbiolo. Abbonda inoltre il trappo in valle dell'Adige fino a Trento, e trappica è la base del monte Bondon, il quale custeggia l'Adige a tramontana di monte Baldo. Scopresi pure frequentissimo negli altri monti veronesi come in valle Pulicella ai dorsi di S. Fiorino presso Miazzurega, e a Marano, come pure a Monte Larzano sopra Avesa, in Valdonega presso Verona, a Lavagno, alla Croce presso Chiesa nuova, alle Scandole, alla Montagna dei pezzi sopra le Scandole, a Tinazzo, presso monte Zevola, presso Badia Calavena, alla Ghiazza, a S. Bartolommeo, al Progno, a Rovere di Velo, a Montebello, e basalto amorfo è il vertice di monte Tomba, basalto colonnare quello di Bolca alto dalla sopraffaccia del mare metri 946,42 . Dal che si scorge, che le rocce trappiche esistono ad ogni altezza de' nostri monti, incominciando dai cento metri all' incirca dal livello del mare come in Valdonega, e a monte Larzano, ed elevandosi ai mille e seicento in Campo dell'Altissimo di 
monte Baldo, e a forse mille e novecento ( $\mathrm{I}$ ) in monte Tomba .

La vachia e il tufo sono sovente coperti dalla roccia calcare, e occorre ben anco l'alternativa ripetuta di strati calcari zeppi di petrificazioni con altri d'argilla e di vachia, o di tufo vulcanico, che contengono pure corpi marini avviluppati entro al loro impasto come in valle di Roncà, e nella vicina valle di Trissino particolarmente a Montecchio. Alle Acque-negre e all'Artillon di monte Baldo ove la roccia costituente il fianco del monte consta di discoliti di molte fatte con pettiniti, echiniti, ed ostreiti impastati con un cemento marnoso-calcare, di eguale natura è pure il tufo vulcanico, ed evvi sorrapposta la calcare comune, che sull'alta vetta diventa oolitica .

La vachia del veronese non è diversa da quella de' vicini monti. Di colore il più bruno o nericcio, talora bigio cinerino, o verdastro, di frattura terrea, opaca,

( ${ }^{\pi}$ Dico forse mille e novecento, non osando asserirlo senza dubbiezza ad onta che n'abbia eseguita la misura barometrica, perchè alcune bollicine d'aria penetrarono nel tubo in seguito a una violenta cadiuta. Tuttavolta le piante seguenti ch'ivi allignano mi fanno accorto, non guari rilevante esserne la differenza:

Achillea Clavennæ.

Anemone alpina.

Arenaria striata.

Athamanta cretensis.

Cerastium latifolium.

Cherleria sedoides.

Dryas octopetala.

Grilinm baldense.

Geum montanum.

Juncus trifidus.

Iychnis quadrielentata.

Lycopodium Selaginoides.

Ophrysalpina,

Paclerota Bonarotta.
Pedicularis rostrata .

Phyteumiz comosa.

Pinus Pumilio.

Primula carniolica .

Rhododendron ferrugineuni

- hirsutum.

Salix reticulata

- retusa.

Saxifraga mutata

- - Ponie.

Tussilago discolor .

Valeriana saxatilis.

Viola biflora. 
senza lucidezza; di durezza mediocre; talora fragile; di odore argilloso, di struttura il più cellulosa a cellette rote, e intonacate di terra verde o piene di sostanza d'altra natura costituendo la mandoloide, e talora affatto massiccia e compatta formando passaggio al basalto. Entrano fra i suoi componenti ordinarj l'orniblenda comune e il feldispato, e talora l'augite - pirossena, impastati da un cemento argillo-ferruginoso più o meno abbondante. I nuclei che riempiono le cellette della mandoloide ora sono calcare amorfa - spato calcare, o talco zografico, o quarzo, o calcedonio che talora fa passaggio al semiopalo come ne' monti di Badia Calavena verso Bolca, o stilbite, o mesotipe, o analcimo. Di peregrina bellezza sono le mesotipi in grossi prismi quadrangolari, riuniti in fascetti disposti a rentaglio o a stella, di aspetto limpido vetroso o bianco, e talora farinoso, che si rinvengono alle falde settentrionali di monte Baldo tra Tierno e Besagno al luogo detto pais. Sono esse disposte in filoncini qua e là nel tufo vulcanico semiscomposto, ed evvi sovrapposto uno schisto bituminoso con vestigia di carbon fossile, forse continuazione di quello della prossima valle di Sorna. La mesotipe rinviensi anche nel tufo della valle di Sam presso $\mathrm{Ca}-$ stione, come pure nella mandoloide e nel basalto dei monti di Lavagno, ma è assai meno pregevole. A Montecchio in valle di Trissino nelle fenditure della mandoloide avvi bellissime cristallizzazioni di stronziana solfatica. Però un fenomeno ancor più singolare offre Monte Viale sul Vicentino. Rinvengonsi in esso corpi organizzati marini penetrati dalla stronziana solfatica, la quale ora li incrosta, e ricopre, ora li pe- 
netra, e ne riempie $i$ gusci in guisa che modellatasi sulla loro figura assume perfettamente la forma della conchiglia, che si è distrutta. Il celebre Ab. Fortis fu il primo a far cenno di cotali petrefatti; ma non essendo nota all'epoca in cui scriveva la stronziana, ebbe i suoi cristalli per ispato calcare. In seguito il Conte Marzari-Pencati valente mineralogo, eseguendo di commissione del Governo italiano una collezione di fossili per le università del Regno, fe' conoscere l' errore del Fortis. Il Sig. Castellini di Castril Gomberto fervido amatore di mineralogia vieppiù ne diffuse la conoscenza, offrendo in dono ottimi esemplari a molti naturalisti .

La vachia o è stratificata, o più sovente rinviensi in grandi masse ammonticchiate, o si conforma in palle di varia grandezza sferiche o schiacciate siccome alle Scalette in monte Baldo, a Tinazzo, presso Chiesa nuova, a Roverè di Velo nei monti Lessini, a Bolca, alla Calvarina in valle di Roncà, a Montecchio e Castel Gomberto in valle di Trissino. Le palle appajono formate di strati concentrici, e constano sovente di trasso o tufo basaltiro, vale a dire di frantumi o ciottoli di basalto impastato da un cemento argilloso, come ò veduto alle Scalette e alla Croce in monte Baldo, e alla Calvarina, a Bolca, e a Montecchio .

Il basalto trovasi sempre prossimo o in continuazione della vachia e della mandoloide. È d'un colore nero grigio più o meno carico, opaco, senza lucentezza, più o meno duro, difficile a spezzarsi, di frattura ineguale scagliosa, come ferrea e ben anco concoide. Fra i suoi componenti si distinguono l'orni- 
blenda e l'olivina. Forma esso ora grandi masse informi come ne' monti Baldu, Tomba, Lavagno (I), ora si conforma in colonne di bellezza meravigliusa. Celebri in tutta Europa per la loro disposizione e r'egolarità sono i basalti de' Panarotti presso S. Gio. Ilarione e d'altri luoghi nella valle di Roncà, ove sono più o meno inclinati all'orizzonte, quelli del vertice o purga di Bolca, e quelli della valle degli Stanghlıellini di Vestena, ove si scorgono perpendicolari. Cotali prismi sono il più esagoni, talora pentagoni, quadrangolari, e rare volte triangolari. Però vengono questi superati per la varietà e grandezza da tuelli che mi vennero veriuti a Trissino, ove esistono prismi di quattro sino a otto angoli, del diametro di mezzo metro, altri di settanta sopra cinquanta centimetri, altri di ottantadue metri sopra trentaquattro. A Trissino pure venne scoperto il basalto in tavole.

Terre gialle e rosse stratificate irregolarmente esistono in varj luoghi de' nostri monti, come alle falde di monte Baldo presso Pilcante, in Verona stessa, a Montorio, ad Avesa, a Marcellise, al Ponte di Veja, a S. Bartolommeo, le quali tutte forniscono alla pittura colori vivaci. Ma rinomate sono le miniere di terra verde o argilla veronese o talco zografico. Giacciono nella mandoloide e nella pozzolana della parte settentrionale di monte Baldo sul pendio d'una profonda

(1) Allorchè neğli anni addictro progettatosi di ristabilire il famoso arcu de' Gavi si scoperse la base dell' Arco e un pezzo d'antica struda ronuana, videsi ch' era questa lastricata cou ciottoli di basalto. Poichè i monti basaltini più prossimi a Verona sono que' di Lavagno, è cosa al tutto verosimile, che da essi abbiano tratto i Romani il basalto occorrente. 
valle intersecata dal torrente Aviana. Secondo l'analisi di Klaproth consta l'argilla veronese di

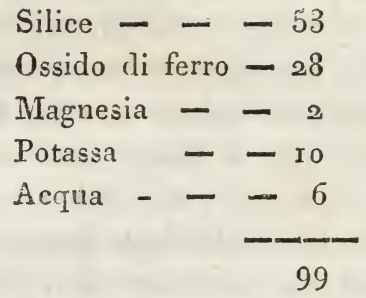

Secondo lo Sternberg e il Meyer contiene anche del Manganese. I filoni sono circondati da verde e da azzurro montano misti a piriti di ferro. In minor quantità si rinviene talco zografico anche presso Castione, e in monte Bolca, e a Lavagno.

Il ferro e il manganese sono i soli metalli, che finora vennero rinvenuti nella nostra provincia. Trovasi il manganese ossidato metalloide alle sette fontane presso Alcenago. Una miniera di ferro, di cui furono incominciate le escavazioni nello scorso secolo ed ora abbandonate per mancanza di co mbustibile, esiste in valle di Botte poco lungi da Brentonico, ed altra per la stessa ragione obbliata avvi alla Ferrara dello stesso monte Baldo, ove si addittano tuttora le reliquie delle fuciue poste sul torrente, le quali anticamente diedero il nome al villaggio. Vestigia di ferro ossidato lenticolare furono scoperte in monte maggiore, di Baldo, e ferro solforato trovasi abbondantemente sparso nella calcare dei colli .

Fra i fossili della provincia rammenteremo innanzi tuttơ la calcare di Lavagno e di Mazzurega, che serve a lastricare le strade di Verona, e che contiene minuti pezzi di quarzo costituendo di tal guisa un' are- 
naria $\left({ }^{*}\right)$. Non è molto dissimile dalla cosi detta piétra forte onde si selciano le strade di Firenze, e che consta di squamette di mica, e granellini di quarzo . Altra arenaria stratificata bruna costituisce il letto del Litantrace della valle dei Prusti sulla sponila del torrente Illasi; e consta di mica, quarzo bruno, e feldispato con nocciuoli di calcare bianca laminare. Le volte e $\mathrm{i}$ sotterranei delle fortificazioni di Verona sono a luogo a luogo coperte di soda sovente mista a porzione di nitro. In un sotterraneo del Castello S. Felice fu rinvenuta abbondantissima e pura. E poichè il colle vicino, e il terrapieno constano di tufo marno-calcare zeppo di ostreiti, pettiniti, echiniti, nummali, ed altri petrefatti marini, pensa il Cav. Lorgna ch' essa prenda origine dalla loro sostanza. A Valcaregna presso Castione nelle cave di marmo trovasi solfato di magnesia erratico, e cave di solfato di soda esistevano altre volte a Roverè di Velo. Gesso inoltre estraesi a Torri, creta a Marcellise e a S. Floriano, e pietre focaje in copia grande al Cerro .

Avara non ci fu la natura anche di acque minerali . $\mathrm{Vi}$ ò già fatto menzione delle acque di Sermione. In valle di Botte in monte Baldo avvi un' acqua minerale fredda, che meriterebbe un' analisi diligente. Manca pure un'esatta analisi d' altra acqua minerale fredda, che zampilla presse Roverè di Velo a fianco d'un monte, la cui roccia principale sono la vachia e il tufo vulcanico con carbonato calcare e solfuro di ferro. I!

(I) Chiamasi volgarmente masegna, nome che i Padovani danno a un porfido particolare che costituisce in molta parte. gli Euganei, e che serve a lastricare Ic strade di Padova, e che per avventura è una lava. 
Sig. Bozza da cento libbre mediche ottenne una qưantità discreta di gas acido carbonico e di carbonato di calce, mezzo grano di solfuro alcalino, ed $8 \frac{1}{3}$ di ossido di ferro.

Una sorgente termale fu scoperta al cadere dello scorso secolo in valle Pulicella, al lato occidentale del palazzo Roveretti, presso il villaggio Domejara, lunghesso la strada di Germania, a nove miglia da Verona. Il Conte Roveretti desiderando possedere una fonte presso il suo palazzo ebbe ricorso al Sig. Pennet, che viaggiava in Italia. Accondiscese questi di buon grado, e a lato al suo palazzo indicò una polla d'acqua profonda metri 6r , 7246 (piedi veronesi 180). Si scavò tosto il suolo nel luogo indicato sino alla profondità di metri 60,50 , ed ecco scaturire un'acqua calda, che si eleva all'altezza di quattro metri. Qualche anno dopo venne ad asciugarsi affatto la sorgente, e rimase asciutta per più mesi : e già trattavasi di scavare il pozzo ivi costruito per altri cinque piedi ( metri 1,7145 ), quando tornò di nuovo l'acqua qual prima, ne più scomparve. Giace tale sorgente a meriggio di un colle intieramente composto di calcare secondaria sparsa di echiniti e d'ammoniti, la quale a luogo a luogo riceve pulimento. Le più prossime vestigie di materie vulcaniche sono quelle poste sopra Mazzurega a oltre due miglia in linea retta fra i monti . I vegetabili ch'ivi allignano sono quelli degli altri colli Veronesi. Il fondo d'onde zampilla la sorgente è elevato dalla superficie del mare metri $7^{\circ}$, posta. la base del palazzo misurata barometricamente di metri 130 . La temperatura dell' acqua appena estratta dalla fonte è in ogni stagione di gradi 34 sopra los 
zero Reaumuriano, e il termometrografu del Beilani da me calato al fondo del fonte segnò gradi 35. La trasparenza, il colore, l'odore, il sapore sono egualissimi a que' dell' acqua comune di sorgente. Il peso specifico ai r 6 gradi sopra lo zero è più di mezzo centesimo di quello dell' acqua distillata, e all' incirca eguale a quello dell' acqua di varj pozzi di Verona . Conservata per più giorni nei recipienti alla temperatura di 16-1 8 gradi + o rimase trasparentissima, ni formò deposito veruno. Esposta al fuoco prese a bollire due minuti prima dell'acqua distillata .

Io aveva deliherato istituirne un'esatta analisi, ma dal cimento d'induzione o analisi preliminare essendomi avveduto della scarsezza e tenue quantità de' principj che contiene, non ò posto cura a proseguirla con quello scrupolo ch'era mestieri, onde precisarne indubitatamente la proporzione. Il risultamento degli sperimenti fu il seguente.

Una porzione d'acqua lasciata raffreddare quindi fatta bollire per un quarto d'ora rimase trasparentissima, nè fece deposito veruno. Cimentata l'acqua nella fonte e appena estratta e raffreddata, e bollita tanto colla tintura di tornasole che con quella di curcuma non offerse cambiamento. Lo stesso uccorse cogli acidi solforico, nitrico, muriatico, colla tintura di galla, coll' acido prussico, colla soluzione di prussiato di potassa, coll' acqua di Lauro ceraso, e coll'alcoole. Coll'acido ossalico rimase da prima trasparente, ma coll'agitazione si fece a poco a poco lattiginosa. Col nitrato e col muriato di barite avvenne appannamento. Filtrata l'acqua su cui aveva agito il nitrato di barite vi si versò nitrato d'argento, che produsse 
un copioso precipitato bianco grumoso. Filtrata l'acqua stessa già cimentata col nitrato d' argento vi si versó acido solforico, che lasciò l'acqua trasparente nè produsse precipitato. Un precipitato spugnoso si ottenne pure dall' acqua termale, su cui si versò nitrato d'argento, prima che fosse stata cimentata co' sali baritici .

Coll'acqua di calce avvenne leggero intorbidamento. Sul sale precipitato si versò acqua stillata, che non lo disciolse interamente. Vi si aggiunse acido solforico, e non avvenne effervescenza. Sperimenti posteriori ànno dimostrato solfato di magnesia disciolto nell' acqua, e la porzione di sale rimasta insolubile solfato di calce si riconobbe.

Coll'acetato di piombo si ottenne dall'acqua termale un precipitato bianco piuttosto copioso. Col carbonato di potassa e d'ammoniaca divenne lattiginosa, e il precipitato raccolto si disciolse con effervescenza nell' acido solforico, e si scoperse un carbonato di magnesia .

Tali sperimenti ed alcuni altri che per brevità io lascio ne rivelano, che 1'acqua termale di Domejara non contiene nessun gas nè acido libero, e che i sali in essa disciolti sono una tenue quantità di solfato di magnesia, e una maggiore di muriato di magnesia . Dall' osservazione dei precipitati ottenuti con varj reattivi inclinerei a stabilire, che in cento libbre mediche d'acqua termale avvi di

Solfato di magnesia grani 30

Muriato di maguesia grani $7^{\circ}$ 
Dall' addottianalisi pertanto nom comprende, debols dover essere la virtù medicinale di tale acqua, e che le guarigioni mercè lei ottenute di alcune artritidi, reumi cronici, ed erpeti voglionsi ascrivere principalmente al calore del bagno con essa fatto.

Dalle falde di due colline di vachia sovrapposta alla calcare conchiglifera stillano le acque termali di Caldiero, celebri fin dai tempi di Augusto e che nel terzo consolato di Petronio Proho ebbero il nome di fonti di Giunone. I Veronesi al finire del secolo 15. mo le ristaurarono, ma tuttavolta sono ora decadute dall'antico splendore. Conservano esse in ogni stagione la temperatura di 22 gradi. Il loro peso specifico è di roo5, e l'analisi di 100 libbre mediche istituita dai Dottori Zenone Bongiovanni e Matteo Barbieri à rivelato

Gas idrogeno solforato in quantità mediocre

Carbonato di calce - gr. 8 ?

- - di magnesia - - gr. I7

- d'allumina - - gr. I00

Solfato di soda - - gr. 32

- di calce - - - gr. 30

- d'allumina - - - gr. 2 I

Muriato di soda marziale - gr. 45

- di calce - - - gr. 15

- di magnesia - - gr. 9

Terra silicea - - - gr. 8

Ossido di ferro $-\quad-$ gr. 6

gr. 280

Finalmente farò un cenno del litantrace o carbon fossile, che a larga mano diffuse la natura sui colli 
e monti Veronesi. E ben è sventura che in tanta scarsezza di combustibili giaciansi neglette cotali miniere, mentre il luro scavo e salverebbe i pochi boschi, che ancor rimarigono pei bisugni delle arti, e diminuireblue l'alto tributo di danaro; che la provincia Veronese paga annualmente al Tirolo per la provvisione del cumbustibile.

Monte Bolca, questo rinomato cimitero d'infinite fatte d'esseri organizzati, e sede di meravigliosi fenomini geologici, offre ben anco un suolo sovrammodo dovizioso di litantrace schistoso e piceo ottimo a far bollire prontamente il ferro. Sopra la calcare, che costituisce la base e l'ossatura del monte, e che racchiude $\mathrm{i}$ celebri ittioliti, riposa una infinità di strati paralleli di litantrace alquanto inclinati all'orizzonte nella direzione del N.O. al S. E. coperti e intersecati da trappo vulcanico, e in alcuni luoghi a immediato contatto del basalto in rozzi prismi paralleli, di cui abbiam già fatto cenno, e che li ricopre e costituisce il vertice detto la Purga di Bolca. Lo schisto bituminoso serve di matrice.

Altra ricca miniera di litantrace schístoso ottimo a far bollire il ferro meglio e più presto d'ogni altro, siccome rivelarono gli sperimenti del Cavalier Amoretti e quelli istituiti nelle fonderie militari, trovasi alle falde settentrionali-orientali di monte Baldo sulla sponda destra del torrente Sorna inferiormente al villaggietto Train. Varj indizj di litantrace dansi a vedere presso S. Gio: Ilarione; e sul pendío del monte Calvarina nella stessa valle di Ro ncà, al luogo detto la casa dei Rossetti, esistono strati di schisto argillo-bituminoso in isfoglie sottilissime, cui $\mathrm{i}$ volgari chiama- 
no libri del Diavoio. Alternano essi can istrati di tulis vulcanico giallo-scuro penetrati dal bitume. Finalmente litantrace ottimo piceo esiste alla Ghiazza, lignite filuroso presso Castagnè, lignite terreo alla Ca di David, litantrace schistoso a Grezzana, e in valle Tanara, e in più altri luoghi, siccome vedrassi dalla luella ed utilissima memoria, ch'è per escire alla luce Sui combustibili fossili dclla provincia Veronese del sullodato Conte Ignazio Bevilacqua Lazise.

A compimento di questo abbozzo geologico debbo aggiungervi, che parecchi nostri colli constano ora di tufo calcare o mattajone, ora di breccie o puddinghe composte di ciuttoli, e frammenti calcari più o meno roluminosi, impastati in un cemento calcare, coi quali ávvi talora dei sassi di porfido e di granito. Tali sono 'ruelli che dividono la valle di Caprino dalla valletta di Rirole, come pure la catena che da Garda va declinando verso Peschiera. Di puddinga sono pure alcuni gioghi meno elevati di monte Baldo, come in valle Basiana, ove risultano di frammenti più o meno voluminosi e angolosi di calcare impastati da un cemento calcare o marnoso; e il fianco settentrionaleorientale dell' Altissimo cii monte Balło sopra Brentonico consta di ghiaja e di sabbia calcare, sicchè offre frane e scoscendimenti considerevoli. Tutta l'alta pianura Veronese infine è quasi interamente costituita d'alti banchi di sassi calcari, porfirici, schistosi, e granitici, di quarzo, di focaja frammisti a sabbia, ad arenaria, a creta e argilla. L'Adige e i torrenti, che giù precipitano da'monti, ne discuoprono a luogo a luogo i diversi strati. Questi ultimi fenomeni par cosa ragionevole attribuire alle varie catastrofi, cui ebbe a 
soffrire il nostro fiume; e sovrattutto alI' epoca che vennero spaccati i monti della Chiusa.

Dalla esposizione fattavi della struttura di monte Baldo voi vedete, mio celebre amico, che non molte cose presenta atte a risvegliare la curiosità del geologo. Che se noi prendiamo ad esaminare gli animali che lo abitano, poco rilevante comprenderemo esserne pure la disamina. Niuno avrene che non sia nei prossimi monti o nel piano Veneto-Lombardo, salvo l'ordine degli insetti, de'quali peregrine specie si rinvengono. Vi ò già fatto cenno degli augelli e dei serpenti nostri. Niun quadrupede particolare abita il monte Baldo. Il solo Lupo (Canis Lupus) vien talora a infestare gli armenti, e l'Orso (Ursus Arctos) e la Camozza (Capra rupicapra) dopo la distruzione delle spaziose selve cercarono altrove più sicura stanza. Quello, onde va celebre questo monte, voi ben sapete essere le infinite e rare piante che alberga. Le sue diverse plaghe, altre apriche e aduste, altre eternamente ombreggiate, le sue valli profondissime e deserte, le sue balze sublimi fanno sì ch'ivi crescano vegetabili di famiglie e di climi disparatissimi. Questa è la cagione ond'è mestieri percorrerlo e nelle diverse stagioni e moltissime volte. Io verrò descrivendovi le varie parti; e ponendo mente allo scopo principale de'nostri viaggi, nutro frducia che non vorrete appormi una soverchia distinzione di luoghi.

Coloro che partono di Verona per due strade possono giungere alle falde di monte Baldo. Una è la via di Germania, che si abbandona valicando l'Adige al di là della Chiusa, e giungendo a Brentino, piccola terra edificata alle radici orientali. Di là per la via 
detta delle scale si ascende alla Corona. Giace questo Santuario in una gola del monte sull'ignudo fianco d'una rupe torreggiante. Guidano ad esso un tortuoso sentiero chiuso fra folte selve d'alberi e d'arbusti assai piacevoli a riguardarsi, e secento e più scaglioni nel sasso scavati, e alternanti con frequenti piani onde riesca meno disastrosa l'ascesa. Fra le fessure di cotali balze germogliano rare stirpi di piante. Presso la sommitì del giogo avri una fonte graziosa, le cui acque gelidissime porgono grato ristoro a quelli che accorrono a visitare quell' sugusto ricetto di solitudine. Alquanto più alto sorgono alcuni casolari detti i Coltri e i Crosati. Da essi piegando verso settentrione si giunge alla Ferrara; ma proseguendo verso ponente ne si presentano le ubertose praterie del Prabasar e la valle Basiana ricca di pascoli e di selvette di Noccioli, di Lamponi, e di Citisi e simili piante fruticose. Sopra questa giace la valle Fredda, la più ferace fra le vicine. Altra valletta posta a mezzodi chiamasi Ime . Però abbandonando la valle - fredda incontrasi a tramontana il Campedello, quindi la Ferrara, villaggio composto di molti gruppi di case e capanne vagamente disposte nel fondo d' una vastissima valle, fiancheggiata a ponente dal maggior dorso del monte, a oriente dal vertice dell' Albaré. I contorni della Ferrara sono ricchi di prati e di pascoli, e intersecati da rigagnoli, che tutti si congiungono nel torrente che giù precipita dai dirupi della Corona. Un profondo ed aspro burrone di questa valle dicesi valle orsa. Progredendo in retta via verso settentrione si ascende al Cambrigar indi in Noveza. Questa parte eminente divide come in due il monte, sicchè le ac- 
74

que, che discorrono dal pendio meridionale, fluiscono nel rivo della Ferrara, e quelle da settentrione nel torrente Aviana. Noveza parte è piena di pascoli feraci, parte è selvosa e dirupata, e in questi dirupi rinvengonsi copiose piante. Poco oltre a Noveza avvi il Campione, e il limpidissimo rio delle Acque-negre, ove altre volte era il confine della repubblica Veneta. L'eccelso dorso di Baldo costeggia a ponente tutti questi luoghi, ed i rivolgimenti del di lui fianco orientale sono determinati con proprio nome. Partendo da valle - fredda il primo luogo chiamasi la Lonza, ove è una valle angusta e inaccessibile detta valle Brutta Segue poscia la valle Losanna e il Sassetto e la Fontana del di lui nome situata sull'alta pendice del monte di fronte al Campione.

Al rio delle Acque-negre tre vie ne si appresentano: l'una a destra guida a monte Gambon, al CerBiolo e alla parte ultima e più orientale di Baldo detta i Lavaci. Per quella di mezzo lungo il ruscello si sbocca al pian della cenere, e alla selva d' Avio. La terza a settentrione scorge all' ampia valle d'Artillon e ai Dossioli, ove un tempo esistevano antiche foreste ora in gran parte divelte. Dall'Artillon si fa passaggio ai prati di Brentonico e del Tredespin. In questi monti era anticamente l' ampia selva detta de'Brentegani rar.mentata dal Pona nel suo Monte Baldo descritto, e che nell' edizione latina chiama sylva Brentonicensis. Abbandonando poscia a man manca gli Zocchi, Navene, e Tolghe, quindi Canalette, e l'Altissimo si scende pel luogo detto le Scalette, ove in due si parte il sentiero. L'uno guida per i Pianetti, Pozza Ferrera, e S. Giacomo ai villaggi Bren- 
tonico, Castione, Tierno e alla valie pereorsa dal torrente Sorna, luoghi situati alle falde settertrionaliorientali; per l'altro orientale si scende per la valle del Tretto e il sentiero de' soppiadori lungo il torrente Aviana alla terra Avio.

L'altra strada che da Verona conduce a monte Baldo è quella di Caprino, dal qual villaggio per varie viottole si può ascendere. Una di queste detta la Costa yei cani guida ai monti della Corona. Per l'altra più meridionale detta via dei Lumini, chiusa fra il giogo di questo nome e il Piore si giunge alla valle Ortigara, dore ne si parano lieti pascoli e boschi di Faggi spaziosi, che colla loro opacità fanno gradito contràsto all' amenità de' vicini prati. Inferiormente all' Ortigara ànnovi le praterie e lo selve di Pra-lungo e di Brenzone. Ma dall'Ortigara ascendendo in via retta fassi incontro l'erboso ed ampio durso Costa bella, a destra del quale giace la valletta di Navole, d'onde si scende in valle-fredda. Nel pendio dı Navole occorrono i sassi di valle fredda, luogo caro ai Botanici per le peregrine piante ch'ivi allignano; e progredendo alquanto rinviensi la fontana di Navole, fonte meravigliosa si per l'altezza sua, che per la copia e freschezza delle sue acque. Una valletta a destra della fonte porta il nome di valle del Bastion.

Da Costa - bella scendendo alquanto per la pendice occidentale entrasi nell' erbosa valle Vaccaria innaffiatis dal grazioso fonte di Brigaldello o delle Buse. Da valle-vaccaria dopo lunga via si passa all' ampia foresta di Piombe. Ma pigliando camınino lungo il dorso Costa-bella si ascende all'altissime cime di Baldo dette monte maggiore. Disastroso però è il tra- 
gitto per l'alta vetta al di là del giogo Coval santo, ond'è mestieri deviare alquanto sul fianco orientale . Lungo la via veggonsi da occidente valli profondissime da sassi e da rupi ignude circondate, nel profondo delle quali alberga eternamente la neve. Sono esse la valle delle buse, le valle delle pietre o di monte Maggiore, la valle delle ossa, la valle $d i$ S. Zeno, la valle grande o fonda o dritta anche chiamata, la valle Orzera, la valle Finestra, e la valle delle Pozzette, selvagge tutte, nè da niuno per avventura frequentate fuor dai Botanici. In questi gioghi difficili e in queste valli allignano le più rare piante alpestri . Però superate le sublimi vette di monte Maggiore, difficile sovrammodo e periglioso riesce il tragitto nelle seguenti, formate di nude punte e di scoscese balze, e fiancheggiate a ponente dalle dette valli Orzera e Finestra. E però trascorso il Sassetto miglior consiglio è abbassarsi sul fianco orientale fi no all'Artillone, ove le cime si fanno meno erte, e a mano a mano s'appianano, e vengono erbose. Per queste facili vette movendo $\mathrm{i}$ passi si giunge agli ubertosi prati e alla maestosa selva di Malcesine e alla bocca di Navene, d'onde si discende al Benaco. Al di qua di Navene s'incontrano a oriente i pascoli dei Zocchi, e a occidente l'asprissimo sentiero di Ventrar (se sentiero può chiamarsi), i di cui dirupi generano peregrine piante. Oltre Navene ànnovi i pascoli di Tolghe e Canalette, e il giogo più settentrionale di Baldo detto l'Altissimo già per noi rammentato.

Eccovi, egregio amico, la topografia di monte Baldo. Porrò fine a questa lettera adducendovi il nome delle specie da me raccolte e in questa e nelle molte altre 
gite da me intraprese, e vi agginngerò il luogo dov' esso allignano, e l' altezza dial livello del mare. Di tal guisa io v'offrirò un saggio di geografia botanica. In ò diviso la geografia botanica Veronese e delle prossime provincie Vicentine e Bresciane in tre regioni o zone, desumendone i confini principalmente dagli alberi, come quelli che concigliano un aspetto proprio alla vegetazione. La prima, che chiarno regione collinesca - delle Querce e de' Castagni, aggiunge a incirca settecento metri. La seconda, la regione montanesca o dei Faggi e delle Conifere, Pini, Abeti, Larici, s'eleva dai settecento ai mille trecento metri. La terza, la regione alpina o dei Mughi e dei Cembri, dai mille e trecento arriva fino alle nevi. La regione sotterranea e la nevosa, quella che incomincia, questa che pon fine alla vegetazione, ambedue abitate dalle crittogame, mancano alle provincie nostre. Perù rispetto alle tre regioni ammesse io non debbo tacervi, che $\mathrm{i}$ confini per me stabiliti non sono al tutto irremovibili, avrenendo qui pure come altrove, ch'ora si elevino, ora si abbassino secondo la diversa plaga. La Carice baldense, poniam caso, pianta montana e ben anco alpina fu da me colta fiorente ai primi di aprile sulle rupi ventose della regione collina di Limone al Lago . Nelle rupi della Corona di monte Baldo meno alti di 800 metri, ma esposti a bacío cresce la Puellerota Bonarotta erba alpina; laddove nei boschi a meriggio di Chiesa-nuova nei Lessini, elevati a più di roometri vegetano le Querce. Cosi nei contorni di Schio e di Recoaro all'altezza di dugento in trecento metri nascono l'Arabis alpina e l'Arabis ovirensis, la Cardımine impatiens, l'Euphorbia syluatica, la Daphne 
Laureola ecc. piante montane. Nella valle dei Ronchi sopra Ala, e alle falde occidentali-settentrionali di monte di Baldo sopra Malcesine ò veduto crescere entro la regione collina l'Arbutus Uva ursi col Rhododendron ferrugineum, e Rhododendron hirsutum cogli Abeti, coi Pini, e coi Faggi, piante montane, e le prime ben anco alpine. Fra gli scogli bagnati dalle acque del Benaco, ma esposti al soffio incessante d'aquilone, tra Malcesine e Navene, tra Riva e Limone crescono la Daphne alpina, il Cytisus purpureus, e lo Spartium radiatum, il quale veste i dirupi di valle-fredda, dell'Artillone, e dell' Altissimo di monte Baldo, e le parti più alte de' monti Pertica e Sumano. Le sponde benacensi Bresciane all'opposto, situate a levante-meriggio fino all'altezza di 150 metri dal mare e di 70 sopra il Lago, sono coperte di Ramerino, di Leandri, di Fichi e di Olivi spontanei, di Melagrani selvatici, di Agave, di Allori. Che se prenderemo a esaminare la vegetazione del Tirolo e del Friuli scorgeremo vieppiù crescere le eccezioni. Dalle quali cose n'emerge, non essese la rarefazione dell' aria che determina l'abitazione delle piante, com' altri pretese. E comunque non neghi affatto $l^{\prime}$ influenza della natura del suolo e della quantità dell' acqua che cade annualmente dal cielo; pure la temperatura è quella che esercita la principale azione sull' abitazione delle piante.

Non reputo inutile avvertire, che molte piante crescendo volgarissime in più parti di monte Baldo iu non ṇe ripeterù il nome ogni volta. Aggiungerò alcune note alle piante novelle $o$ pregevoli o che meritano dilucidazione. Seguirò la nomenclatura del Will- 
denow per le Fenerogame e per le Felci, quella dell'Hedwig o del Bridel o dello Schwaegrichen nei Muschi, del Roth nelle Epatiche, del Vaucher o del Dillwyn nelle Alghe, dell'Acharius nei Licheni, e del Persoon e Decandolle nei Funghi.

Dalla valle di Caprino pertanto sino ai Masi e al cosi detto pra-bestemmia sotto pra-lungo all'altezza d'incirca 700 metri crescono le seguenti, che si rinvengono pure nella più parte dei colli e nell'alta pianura Veronese.

Acer campestre .

Achillea Millefolium

\section{- tomentosa.}

Adonis anuua. A. xstivalis et $\mathbf{A}$. autumualis $\mathbf{L}$.

A. caule glabrc superne sulcato, foliis multifido-linearibus, petalis 3-8 oblongoovatis . Mihi.

L'Adonis aeslivalis, e l'Adonis autumnalis non scno specie ma varictà; perocchè fra le biade si rinvengono individui coi fiori a otto petali grandi, altri con cinque minori, altri con tre, quattro, sei, sette di varia grandezza, e dai semi raccolti da essi ò ottenuto pressochè tutte le suddette varietà .

Aecidium clematitidis

- confertum - DeC euphorbiae.

Aegilops ovata. Agaricus edulis . Agrimonia Eupatorium . Agrostemma Githago . Aira caryophyllea _- cespitosa

Ajuga Chamaepithis - genevensis reptans.

Alchemilla Aphanes.

Allium angulosum

\section{- carinatum}

- paniculatum

- rotundum

- vineale.

Alnus glutimosa.

Alopecurus agrestis

- geniculatus.

Alsine media.

Althaea cannabina.

Alyssum calycinuma

- paniculatum

_- Sativum.

Amanita caesarea. Fra i Castagni al luogo detto i ilasi. Amaranthus Blitum

—_ retroflexus

- sylvestris.

Anagallis arrensis $\alpha$ fonicea

- - $\beta$ corulea .

A. caule diffuso, foliis ovatis subtus punctatis, calycis laciniis lanceolatis. Milii.

II solo colore della corolla nelle due varietì è cosiante nella seminagione, gli allri caratteri desuniti dalle foglie, dai peduncoli, dalli forma e contorni della corolla, dal calice variano tntti; e is raccolto ben atro esemplari a foglie terne, 
quaderne tanto delía varietà cerulea che della fenicea . Anchusa italica - officinalis.

Andropogon Gryllus

- Ischaemum

- halepensis.

Anethum Foeniculum .

Angelica sylvestris.

Anthemis arvensis

- Cota

- Cotula

- tinctoria. (Buftalmo del

Mattioli. Pona bald.ital. 4.) Anthericum ramosum.

Authoxantum odoratum. Anthyllis Vulueraria. Apargia autumnalis.

Apargia crispa

- hastilis .

Arabis thaliana.

Arctium Lappa.

Arenaria serpillifolia

- tenuifolia ( $\mathrm{Alsine}$ tenui-

folia. Segu. Ver. 1. 418.tab.

VI. fig. 2.)

- - $\beta$. pentandra (AL-

sine pentastemon, gramineis

foliis, geuiculata.Segu. $V e r$.

III. I 75 .

Aristolochia Clematitis .

Artemisia Absyntium

- campestris

- camphorata.

A. caule erecto; foliis caulinis pinnatis, incanis, pinnis subtrifidis linearibus, floralibus indivisis, linearibus; panicula virgata; anthodiis glohosis pedunculatis nutantibus; peranthodiis incanis. Mihi.

Artemisia camphorata $\beta$. A. subcanescens. W. Enum.

L'Artemisia camphorata e l'Artemisia subcanescens W. volgarissime in tutti i nostri colli sono varietà, siccome mi fecero accorto e l'osservazione instituita per più anni sulle piante spontanee, c la loro coltura nell' Orto di Verona. Le foglie ora sono yuasi verdi affatto, ora bianchiccie, ora al tutto bianche presentando un abito diversissimo. Il Seguier (Ver.II. p. 173.174) sotto i num. I. 3. à descritto la varietà $\alpha$ e $\beta$. La prima ò veduto coltivata in qualche città d'Italia sotto il nome improprio di Abrotano.

Artemisia vulgaris .

Aram italicum .

Asclepias Vincetoxicum .

Asparagus acutifolius

_— officinalis.

Asperugo procumbens.

Asperula arvensis

- cynanchica.

Aspidium Filix mas.

Asphenium Adianthum nigrum

Ruta muraria

Trichomanes

co. Pona bald. ital. If́2. 250. )

Aster Amellus,

Astragalus Cicer

- glycyphyllos

- Onobrychis.

Athamanta Cervaria

- Libanotis

Oreoselinum.

Avena fatua

- flavescens.

Ballota nigra.

Barbula ruralis. Brid.

Berberis vulgaris.

Betonica officinalis .

Bidens tripartita

- - tripinnata.

Biscutella apula .

Boletus edulis. Fra i Castagnị ai Masi.

Borrago officinalis . 
Borrera chrysopluthalma e.

Sui rami dell' Olivo e dello Spimbianco .

\section{- tenella.}

Prassica Erucastrum

\section{- Napus}

- orientalis.

Briza media.

Bromus arvensis

- - erectus

- mollis

- squarrosus

- stcrilis.

Sryouia divica.

Bryum argenteun.

Dunias Erucago.

Puphtalmum grandiflorum

- spinosum.

Bupleurun Gerardi

- Odontites. Ai Masi.

rotundifolium.

Calendula arvensis.

Calluna vulgaris .

Campanula glomerata

_- persicifolia

Rapunculus

- - sibirica

Spcculum

- Trachelium.

Cardamine hirsuta.

Carduus defloratus

- nutans

Cares digitata

- flava

- hirta

- Michelii

- pillulifera

- praecox. (Cyperoides rerna, caule rotundo triquetro, spicis seminalibus densioribus, binis rel ternis squamis ferrugineis, obtuse mucronatis, et tamquam in aristulam prolungatis, capsulis turbinatis subhirsutis trilateris. Segu. Ver. I. p. $122 v$ t. I. Gg. 2.)
Carex recurva

- Schreberi

- vulpina.

Carlina acaulis

- vulgaris.

Carpesium cornuum .

Carpinus Betulus.

Carthaums lanatus .

Castanea vulgaris.

Cancalis Authriscus

_- dancoides

- grandiflora

- nodosa.

Caulinia fragilis.

Celtis australis.

Cenomyce alcicornis $\propto$

- epiphylla $u$

- pyridata

- - rangiferina $\alpha$.

Centaurea calcitrapa

- crupina

- Cranus

- Jacea: ramis angulatis, foliis lanceolatis, integerrimis, radicalibus dentatis, peranthodiis scariosis, squamis ovatis apicc jaceris, pappo subnullo. Mihi .

\section{W.}

$\beta$ Centaurea amara.

C. foliis radicalibus lanceolatis subdentatis, summis lincari-lanceolatis integerrimis . Mitic . Cyanoides montana squamata, flore purpurco . Ponted. Diss. 219. Segru. Ver. II. 152. Moren. Hort. sicc.

Io penso col Gerard che la Centaurea Jacea, e la Centuurea amara sieno varietà, mentre i caratteri clie le distinguono sono sovranmodo incostanti. La varietà $\beta$. ossia la $C$. amara è assai volgare in tulta la provincia. II colore della corolla in ambeclue le varietà ora ć por- 
jorino, ora ma piì raraincute bianco.

Centaurea nigrescens

- paniculata

- scabiosa

_ solstitialis

- splendens.

Cerastium aquaticum

- arvense

- viscosum

_ vulgatum.

Cercis Siliquastrum .

Cerinthe minor.

Chaerophyllum temulum .

Chara flexilis o. major. Cliara translucens major flexilis. $V$ aill. par. I 8 . t. 3 . f. 8 .

- - B.minor. Chara trans lucens minor flexilis. Vaill. par. 18. t. 3. f. 9. An species praccedente distincta?

Nella fossa volgarmente pozza ove si abbeverano gli armenti al luogo detto il pra-bestemmià .

—_ vulgaris. Ivi.

Cherianthus erysimoides.

Chelidonium majus .

Chenopodium murale

- polyspermum

Vulvaria ( Tragio germa-

nico. Calceol. Viagg. 12.)

Chlora perfoliata.

Chondrilla juircea .

Chrysanthemum Leucanthemum .

(C. foliis radicalibus spathulatis, caulinisque inferioribus petiolatis serratis, reliquis semiamplexicaulibus lanceolatis, subinciso-serratis. Mihi.

Leucanthemum vulgare. Tournef. Instit. 492. Ponted, Diss. 282. Segu. Ver. I1. 222.

Chrysanthemum Leucanthe- mum. Linn. Willd.Sp.pl.IIr. 3142. Pers. Syn. pl. II. 460. Bellis sylvestris caule folioso major. Bauh. Pin. 26 r.

Bellis major. Fuchs. I 48. Cam. Epit. 635. Blackw. t. 42.

Ch. caule plerumque monanthodio, foliis serratis, radicalibus cunciformibus vel spathulatis, reliquis lanceolatis, squamis peranthodialihus atro-marginatis. Milhi.

Ch. montanum. Linn. Sp. pl.

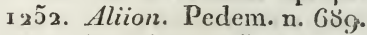
t. 37. f. 2. Jacq. 065. 4. p. ?. t. 9 r. Willd.Sp. pl.III. 2143 . Pers. Syn. pl. II. 460.

Ch. atratum. Linn.Sp.pl. 1253. W. Sp. pl. III. 2142. Pers. Syn. pl. II. 460.

Leveanthemum montanum minus. Tournef. Instit. 492.

Bellis montana minor. Bauh. Hist. III. p. I15. c. ic.

Leucanthenum alpinum majus rigido folio. Tournef. Instit. 492. Segu. Ver. II. 223. Moren. Hort. sicc.

Leucanthemun montanum majus folio acuto. Tournef. Instit. 492. Segu. Ver. III. 28 r. Moren. Hort. sicc.

Bellis alpina major rigido foliø. C. Bauh. P'in. 26 1. Prodr. I20. c. ic.

Bellis alpina major, foliis angustis rigiclis J. Bauh. Hist. III. I 15 .

Bellis montana major folio acuto . C. Bauh. Pin. $26 \mathrm{r}$. Prodr. I2r. c. ic.

Bellis alpina minor foliis non rigidis. J. Bauh. Hist. III. 1 I5.

La varietà $\alpha$ ossia la Bellide maggiore cresce in tutti $\mathrm{i}$ prati e pascoli della provincia ; la varietà $\beta$ io l'ò rac- 
colta in valle-fredda $e$ in altri luoghi di monte Baldo, ove fu parimente rinvenuta da Gasparo Bauhino, dal Pontedera, Seguier e Moreni, del pari che negli Euganei ove fil pure osscrvata da Gasparo Bauhino. Cresce anche negli altri monti Veronesi, Vicentini, Tirolcsi, e Bresciani .

Le descrizioni e le figure del Ch. montanum date da Giovanni Bauhino e dall'Allioni m'ànno persuaso essere que sto varietà del $C h$. atratıun; anzi le defirizioni del $C h$. monlanum slese da Linneo e dal Willdenow meglio si confanno agli esemplari baldensi ed euganei del Ch. $u$ tratum da me colti e dal Seguier e Moreni, che al $C h$. montanum stesso. E pongasi mente che le due figure di Gasparo Bauhino, le quali si riferiscono dayli autori al Chrysanthemum atratum, furono disegnate sopra esemplari raccolti appunto in monte Baldo e sugli Euganei, ciò che a mio credere è una novella prova della identità delle due credute distinte specie.

Che poi il $C h$. alratum e il Ch. montanum sieno varietà del Ch. Leucanthemum m'ànno convinto gli esemplari di quest'ultimo colti nelle monlagne meno clevate, i quali offrono caratteri comuni ai due primi e al secondo. Veggo che rispetto al $\mathrm{C} / \mathrm{h}$. montanum e al C'\%. Lencanthemum fu fatta la stessa osservazione dal Besser nelle sue Primitiac Florac Guliciae
Austriacae part. II. p rg9,, ()t specimina it D. F. Christiani in montosis circa Dulila collectu, quae manifestissime medium inter Ch. Lencanthemum et Ch. montanum tenent has species praceunte Ch. Gerardo, conjungo, ,). Cichorium Inthybus.

Circaca lutetiana.

Clavaria sorniculata .

Clematis recta Vitilba.

Cnicus lanceolatus.

Colchicum autumnale.

Collema nigrescens.

Convallaria Polygonatum .

Convolvulus arvensis

- Cantabrica

- sepium.

Conyza squarrosa .

Coriandrum testiculatum.

Cornus sanguinea.

Coronilla Emerus

- minima

—_ varia .

Corylus Avellana.

Crepis foetida

—_ hispida

- tectorum.

Crocus vernus.

Cucubalus Behen

- Otites .

Cuscuta curopaea.

Cyclamen europacum .

Cynodon Dactylon.

Cynoglossum pictum.

Cynosurus cristatus.

Cytisus hirsutus

nigricans

sessilifolius.

Dactylis glomerata.

Daucus Carrota.

Delphinium Consolida .

Diauthus Armerius (Caryophyllus barbatus sylvestris. Segu. Ver. r. 437. tab. ?. fig. 4.) 
Dianthus atrorubens. (Caryophyllus sylvestris, flore rubro plurimo de summo caule prodeunte. Ponted.Segu. Ver. I. 434. tab.7. fig. 2.)

- carthusianorum . (Caryophyllus barbatus angustifolius, petalis rubris, maculis purpureis et villis circinatim dispositis in umbilico aspersis. Segu. Ver. I. 438. tab. 8.)

- prolifer (Caryophyllus sylvestris, prolifer. Segu. Ver. I. p. 433. tab. 7. fig. x.)

- sylvestris. (Caryophyllus sylvestris flore rubro inodoro, calyce oblongo cum brevibus unguibus. Ponted. Segiz. Ver. I. 435. tab. 7. fig. 3. Armerio terzo del Dodoneo? Pona Bald. ital. II. )

Dictamnus albus (Poligonato di Dioscoride, ovvero Frassinella degl' Italiani . Pona Bald. ital. x2. Dittamo bianco. Calceol. Viag. 9. )

Digitalis ambigua (Digitale gialla di fior grande. Pona Bald. ital. 173. fig. C. )

\section{- Iutea.}

Digitaria sanguinalis .

Dipsacus sylvestris.

Dorycnium herbaceum .

Draba verna (Alysson vulgare, Polygoni folio, caule nudo. Segu. Ver. I. 376. tab. 4. f. 3.)

Echium italicum

- vulgare.

Epilobium angustissimum

- hirsutum

- pubescens.

Epimedium alpinum .

Equisetum arvense

- hiemale.

Erigeron acre

- canadense.
Erodium ciconium

- cicutarium.

Ervum hirsutum.

Eryngium amaethystinum

- campestre .

Erysimum officinale .

Eupatorium cannabinuna.

Euphorbia Chamaesyce

- Cyparissias

dulcis

- falcata

_ helioscopia

_- nicaeensis. ( Tithymalus

foliis brevibus aculeatis. Segu.

Ver.I. p. 154 . t.3. f.r. Pity usa.

Matth. 1258. Calceol. Viag.7.)

- Peplus

- platyphyllos

- verrucosa.

Euphrasia lutea

_- odontites

__- officinalis .

Eronimus europaeus.

Fedia coronata

_- dentata

- olitoria.

Ferula nodiflora.

Festuca ciliata . $D_{e} C$.

_- cristata

-_ duriuscula

- Myurus

- ovina

— pinnata

- pratensis. Schiad. $\mathrm{W}$. Enum.

- serotina (Gramen loliaceum serosinum panicula dispansa.Segu.Ver. III. p. I 46 . tab. 3. fig. 2.)

Fragraria collina

- vesca.

Fraxinus Ornus.

Fumaria officinalis

__ - 3 caule humiliore erecto rigido.

La varietá $\beta$ volgare infra i campi meno fertili coltivati a biade fu deteruninata dal 
Seguier ( Plant. reron. II. P. III ) colle seguenti frasi ripetute anche dal Moreni nel suo crbario , Fumaria minor tenuifolia, caulibus surrectis, flore hilari purpura rubente. Baule pin. I 3 ; Capnos tenuifolia. Clus. hist. CCVIII. c. ic. ,, le quali riferendosi alla Fumaria spicata ànno fatto conchiudere al Linneo che la Fumaria spicata cresca nei campi nostri, lo che non è altrimenti vero. Galega officinalis. Galeopsis Ladanum 'Iethrait.

Galium Aparine

- Bocconci

- Mollugo

- rubrunı verum .

Genista germanica

- ovata

- tinctoria.

Geranium colombinum

- - molle

- robertianum

- sanguincum.

Geum urbanum.

Gladiolus communis.

Grlechoma hederacea.

Globularia vulgaris.

Gnafalium germanicum

- luteo-album.

Grimmia apocarpa

- ovata IVeb. et Moler.

Gypsophylla Saxifraga.

Hedera Helix.

Hedysarum Onobrychis.

Helianthemum marifolium. Milii (II. suffruticosum extipulatum divaricatum, foliis petiolatis oppositis ovatis acutis, snbtus cano-tomentosis, floribus racemosis. Mihi.

Cistns marifolius et Cistus canus. Linn.
Helianthemnm foliis Mrrli mi noris subtus incanis. Segul. Ver. I. p. fiz. Moren. et Bordon. Hort. sice.

Cisto terzo ungarico del Clusio. Puna Monte baldo descritto p. $150 ; \mathrm{e}$

Cisto piccolo con foglia mirtina del Clusio. Pona ivi 2 f́.

- - $\beta$ Cistus anglicus. Linn. H. suffruticosum extipulatum divaricatum, foliis petiolatis oppositıs, oblongis, acutis, subtus cano-tomentosis, floribus racemosis. Milii.

Helianthemum alpinum, foliis Pilosellae minoris Fuchsii. Segu. Ver. III. p. I96. Moren. et Bordon. Hort. sicc.

Il Cistus marifolius, C. canus, $C$. anglicus $\mathrm{L}$. sono varietà d'una stessa specie volgarissima in tutti $i$ uostri colli).

Helianthemum salicifolium (Helianthemum annum humile foliis ovatis, flore fugaci . Segu. Ver.III. 197.t. 6. f.3.)

_- vulgare.

Heliotropium europaenm.

Helleborus foetidus

- - viridis.

Helvella leucophaea .

Herniaria glabra .

Hibiscus Trionum.

Hicracium floreutinum

- murorum

_- Pilosella

_- sabaudum

_- umbellatum.

Hippocrepis comosa .

Holcus lanatus

odoratus .

Hordeum murinum .

Holosteum umbellatun.

Hottonia palustris.

Humulas Luppulus. 
Hydnum coralloides. In trunco Mori albae.

Hyosciamus niger.

Hypericum hirsutum

_- perforatum.

Hypnum arbuscula. Brid. $\mathbf{H}$. alopecurum L. Hedw.

_- cupressiforme

- serpens.

Hypochacris radicata.

Jasione moutana.

Inula disenterica

- hirta

-. Pulicaria

- squarrosa.

Iris germanica .

Jungermannia dilatata

- platyphylla.

Juniperus communis .

Lactuca perennis

- Scariola.

Lamium album

- amplexicaule

-n maculatum

- purpureum.

I apsana vulgaris .

Jatliyrus Aphaca

__ latifolius

- pratensis

- netifolius

- sphoericus.

Lecanora albella

_- milvina

- murorum

- salicina

- snbfusca

- varia

- versicolor.

Lecidea baldensis: crusta imbricata foliaceo-lobata pallide virescente subtus alba fuscescente, lobis minutis crenatis, apotheciis confertis planis incarnatis demum marginatis. Spreng. PI. min. cogn. pug. If. p. 95. Pollin. Pl. nov. vel min. cogn. p. 28 . - callosyuc
Lecidea lapicidá parasema

- Wulfenii.

Leontodon Taraxacum .

Lepidium graminifolium .

Leskea polyanthos

scricea.

Ligustrum vulgare.

Linkia Nostoc

- pulposa Mihi. L. suborbicularis fusca lobata, lobis crassis subimbricatis crenu. latis, superficie centrali apotheciis rufis sparsa. Pollin.

Pl. nov. vel min. cogn. p. 3a.

- verrucosa.

Linaria chalepensis

- Cymbalaria

- minor

_- vulgaris.

Linum catharticnm tenuifolium.

Lithospermum arvense

- officinale

- purpurea-cocruleum .

Lolium arvense. With. Smith.

Schrader.

- perenne

- temulentum .

Lonicera Caprifolium

- X Xlosteum .

Lotus corniculatus.

Lychnis dioica

__ Flos Cuculi.

Lycopus europaeus

_- exaltatus.

Lythrum Salicaria .

Malva rotundifolia

—_ sylvestris.

Marrubium vulgare.

Matricaria Chamomilla.

Medicago falcata

Gerardi

Lupulina

- minima

orbicularis

sativa .

Melampyrum arvense 
Melampyrum cristatum

nemurosum

\section{- prateuse.}

Melica ciliata

- cocrulea.

Ifelilotus oflicinalis $\boldsymbol{x} \mathrm{fl}$. luteo. vulgaris. W. Enum.

21. caule erecto foliolis ovatooblongis dentatis, leguminibus racemosis pendulis suhrugosis oratis, mono-lispermis. Milhi.

Il celebre Willdenow nella sua Fnumeratio horti berolinensis p. 790 d fatto due specie distinte delle due varietà a fior giallo $e$ a fior bianco del Melilotus ofjicinalis, e il carattere principale consiste nel pericarpio disperno nella varieta a fior giallo, monospermo in quella a fior bianco. Ma io ò rinvenuto ora uno ora due semi tanto nell' una che nell' altra. Ambedre sono appo noi annue, e la varietà a fior giallo à lo stendardo senzat vene fosche.

Melissa officinalis.

Melittis Melissophyllum.

Menthat arvensis

- rotundifolia.

- sylvestris.

Mercurialıs annua.

Merulius Cantharellus.

Mespilus monogyna

_- Oxyacantha. Negli spineti e nelle selve.

Muium cuspidatum

- undulatuan Hedsv. M. Li gulatum Brid.

Morchella esculenta

- patula.

Myagrum rugosum

_- striatum Mihi. Papistrum monospermula. Segu. Vcr. I. 36s. Moren. Hort. sice. non Baulh. Piu.

Radix annua ramosa, flavescens.

Caulis sesqui-bipedalis, erectus, ramosus, inferue sulstriatus hirsutus, pilis albis retrorsum asper, superne glaber. Folia alterna, petiolata, obovata, snbhirsuta, scabra, dentata, basi sinuata, caulina media oblonga dentata, ramea lanceolata obtusiuscula suldeutata. Panicula terminalis, composita, racemis erecto-patulis nudis. Flores alterni pedunculati, sepalis palidis, petalis luteolis. Ovarium biarticulatum in calyce sessile. Siliculae erectae, glabrae, striatae, stylo obtuso longiore vel subaequali terminatae, biarticulatae, articulo inferiore stricto abortivo, superiore rotundoovato monospermo.

Provenit etiam in arvig vicis Vallese, Oppeano, Bonavigo, Minerbe adjacentibus.

Il Seguier e il Moreni, che ànno rinvennto la descritta specie presso Oppeano e il Vallese, ore fu pure da mo raccolta, l'ànno determinata colla frase del Bauhino sopraccitata, la quale si riferisce dagli Autori al Myagrum perenue. Anche nell'erbario del Vitman, cui posseggo. esiste col nome di My'agrum perenne. L' à spodita a qualche Botanico mis corrispondente, e fu determinata per lo Myagrum rugrosuin. E di vero a questa specie per la forma del fiore, e della silicietta e pel fusto annualo s' avvicina, tanto pirt che dassi una vas 
rictit notata dal Roth Germ. II. p. $8_{1}$. siliculis minus pilosis. 'íuttayolta oltre delle siliquette affatto liscie ne differisce per le foglie. S' accosta più al My agrum oricntale, secondo quello che puossi arguire dalla frase specifica, non avendo sotto gli occhi un esemplare. Frattanto io determinerò le tre specie $\mathrm{My}_{\mathrm{y}} \mathrm{a}$ grum striatum, Myagrum rugosum, Myagrum perenne colle seguenu frasi:

Mragrum striatum: foliis obovatis dentatis, basi sinuatis, rameis lanceolatis subdentatis, siliculis biarticulatis, monospermis, striatis, glabris.

Myagrum rugosum: foliis oblongis, obtusis, sinuatis, obtuse dentatis; siliculis biarticnlatis, monospermis, striatis, pilosis, stylo brevioribus.

Myagrum perenne: foliis oblongis pinnatifido-sinuatis, dentatis, scabris, summis serratis, glabris; siliculis biarticulatis, monospermis, striatis, stylo longioribus.

Siliculae glabrae, quandoque pilosac.

Myosotis arvensis

-r Lappula .

Neckera crispa

- curtipendula

- viticulosa.

Nepeta Cataria.

Ononis minutissima

- pinguis

- spinosa.

Onopordon Acanthium .

Onosma ,echioides.

Ophrys apifera. (Segu. Ver. III. p. 246 . n. 4. tab. 8. fig. 2.)

- arachuites. (Segru. Ver.
III. p. 244. n. '3. tab. 8. f. I.)

Ophrys aranifera ( Segu. Ver: II. p. 130.13 I. n. 18. 19. 20 . 21. tab. I5. f. I3. 14.)

Orchis Morio (Segu. Ver. II. p. 125. I 26. n. S. 9. tab. 15. fig. 7. 8.)

- piramidalis ( Segu. Ver. II. p. 129. n. 15. 16. tab. 15. fig. I I. )

- ustulata (Segu. Ver. II. p. 123. n. 4. tav. 15. fig. 4.) Origanum vulgare.

Ornithogalum umbellatum.

Ornithopus scorpioides.

Orobanche caryophyllacea .

Orobus vernus.

Orthotrichnm anomalum. Hedw.

O. saxatile. Brid.

- cupulatum. Brid.

Oxalis corniculata.

Panicum glaucum:

- verticillatum.

Papaver Argemone

- Rhoeas.

Parietaria officinalis .

Parmelia aipolia

- caperata

- cartilaginea

- glomulifera

- parietina

- saxatilis

- stellaris

- ulothrix.

Pastinaca sativa.

Peltidea canina.

Peziza lycoperdoides. $\alpha$. latea. De C.

Phleum asperum

- Bohmeri

- pratense

- $\beta$ P. nodosum.

Physalis Alkekenghi.

Picris bieracioides.

Pinpinella magna

Saxifraga.

Pistacia Terebinthus. 
Plantago lanccolata

- - S. P. trinervia, angustissimo folio Bauh. Piu. 189.

major

- media.

Poa anuua bulbosa

- 3 . vivipara

megastachys. Kol. Gaud.

Briza Eragrostis $L$.

—— nemoralis

- pilosa

- pratensis

- - $\beta$. Poa angustifolia.

- rigida Linn.

Polychnemum arvense.

Polygala vulgaris .

Polygonum avicular

- Convolvulus

- Hydropiper

- minus

- Persicaria.

Polypodium vulgare.

Populus alba

- nigra

- tremula.

Potamogeton densum

- crispum

marinum

- natans

Potentilla alba

-

- recta

- reptans

verna.

Poterium Sanguisorba.

Primula vulgaris.

Prunella graudiflora

- vulgaris

- - $\beta$. fl. albis

Prunus Avium

- Mahaleb

- spinosa.
Pulmonaria officina!is.

Pulsatilla pratensis.

Pulveraria incana.

Punica Granatum.

Pyrus Amelanchier

- Aria

- torminalis.

Quercus Aegylops. Sui colli verso Affi, presso la Casa vecchia del Becelli.

-_ Esculus

- pedunculata

- Rohur.

Ranunculus acris

- arvensis

- bulbosus

- Ficaria

- lanuginosus

- repeus.

Reseda lutea

- Phyleuma.

Ragadiolus stellatus.

Rhinanthus Crista galli.

Rhus Cotynus .

Rosa arrensis

- canina

_- pumila. Nelle siepi .

- rubiginosa fl. albo.

Rottbölla monandra.

Rubus cocsius

- fruticosus.

Rumex Acetosa

- Acetosella

- acutus (Ossilapato. Calccol. Viagg. 10.)

- crispus

- obtusifolius

- pulcher.

Ruscus aculeatus.

Ruta clialepensis :

Salix alba

- caprea .

Salvia pratensis.

Sambucus Ebulus

- nigra.

Saponaria officinalig

- Vaccaria.

Satureja hortensis 
Satureja montana.

Saxifraga bulbifera tridactilites.

Scabiosa arvensis

- Columbaria .

Scandix Antriscus Pecten.

Scirpus Holoschoenus

- palustris

- triqueter.

Scleranthus annuus.

Scorpiurus muricata.

Scrophularia canina

- nodosa.

Sedum album

- dasyphyllum

- reflexum

Senecio aquaticus

-.. vulgaris.

Serratula arvensis

- tinctoria.

Seseli annuum .

Siesleria cocrulea.

Sherardia arvensis.

Silene nutans.

Sinapis arvensis

- orientalis.

Sisymbrium Nasturtium

- Sophia

- - sylvestre

- tenuifolium .

Solantim Dulcamata

— miniatum

- nigrum .

Solidago Virgaurea .

Sonchus arvensis

- oleraceus $\propto$ levis

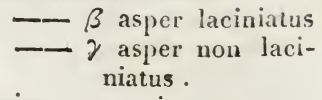

Sphaeria concentrica.

S. subrotunda vel tuberculiformis, nigra, intus stratis concentricis interrupta. Mihi.

In truncis Salicis albae, ct Populi nigrae emortuis.

is una delle specie della massima grandezza, eguagliande. sovente una grossa noce, sicchè pare un Petilupo o $V_{c}$ scia lupaja antica. È nera coriacea esteriormente e levigata: taluno autore però la osservò di color fosco e a superficie inuguale e pustulosa. La sua figura ì assai varia, ora bistonda, ora ovata, sovente munita d'un brevissimo e grosso stipite segnato di rialti circolari, superiormente ampliantesi in tubercoli più o meno pronunciati. La sezione verticale offre tanti straterelli semicircolari o quasi circolari concentrici bianchi, fragili come il midollo di molte fanerogame erbacce, e separati da setti neri più consistenti, i quali sono quelli clie costituiscono i rialti circolari sulla base del fungo . Nei tubercoli maggiori ò annoverato fino a I 8.20 strati . Cotali strati sono tutti eguali, salvo i tre o quattro più esteriori, che a mano a mano vanno assottigliandosi, sicchè l'ultimo immediatamente sottoposto al tegumento è appena distinguibile. Questi inoltre non sono candidi, ma d' un bianco sudicio. Osservata a microscopio composto una sottile laminetta perpendicolare d'uno strato unitamente al suo setto, apparisce composta d'un tessuto membranoso trasparente e segnato da fibrille longitudinali, con alcune trasversali a luoghi indeterminati. Talora dansi a vedere cziandio alcuni punti simili a quelli del tessuto mcmbranoso delle fauero- 
game. Non sono peró gुimto a distinguere in essi puuti il puntino centrale trasparente. Il tessuto membranoso dello strato giunto al setto si fa piu sodo, offre cellette o fibre più titte e si tinge in nero. 'Torna qnindi a farsi soffice e candido costituendo lo strato seguente. La tessitura del tegumento è molto simule a quella dei setti. Unasfogliasottile trasversale di uno strato dà a vedere mercè il microscopio un tessuto membranoso, che si apre in cavità tubulose rotonde od ovate o irregolarmente angolose, le quali corrispondono alle fibre che appariscono sulla sezione verticale. Cotali tubi non sono voti nell'intera loro lunghezza, ma a luogo a luogo sono interseccati da una parete membranosa o cellulosa trasparente, alla quale penso corrispondano le fibre trasverse, cui dicemmo osservarsi sulka sczione verticale. Gli strati estcriori offrono tessnto meno sviluppato e talora indefinibile; dal che conchiudo essere i più giovani. Per ciò che spetta agli organi moltiplicatori io non sono riescito a distinguerne nessuno.

Spiraca Filipendula.

Stacliys annua

- germanica

- palutris

Stellera Passerina.

Stipa pinnata.

Symphytum officinale.

Tamus communis.

Tanacetum vnlgare.

Teacrium Chamaedrys
'Teucrium Chamaedrys. $\beta$.

Thalictrum angustifolium

- flavum

'Thelephora acerina. Sul Noce.

- Lirsula

- purpurea.

Ihlaspi Bursa pastoris

- perfoliatum.

Thymus Acynos

- - angustifolius. Pers.

- Calamintha

- - grandiflorus

- lanuginosus

- Nepela

Serpillum.

Tortula muralis. Brid.

- unguiculata. Brid.

'Tordylium maximum .

'Tragopon prateuse.

Tribulus terrestris.

Trichodium caninum .

'Trifolium agrarium

—_ alpestre

__ angustifolium

arvense

fragiferum

medium

montanum

prateuse

repens

- rubens

- scabrum.

Triticum repens.

Tuber cibarium

- rufum. Mihi. Nelle vigne e nelle selve dei colli presso Colà. T' globosum soliclum, rufum, faeviusculum, arrhizon, interne primum sordide albidum, dem rufescens, venis albis.Pollin.

PI. nov. vel min. cogn. p. 33.

'Tulostoma boreale.

'Furritis hirsuta.

'Tussilago Farfara

_- Pulasites.

Ulmus campestris.

Urtica dioica 
Urtica urens:

Valantia cruciata glabra.

Valeriana officinalis rubra.

Variolaria communis.

Verbascum Blattaria

- Lychaitis

$$
\text { 3. fl. albo }
$$

- nigrum

Thapsus .

Verbena officinalis .

Veronica Anagallis

- arvensis

- Beccabunga

- Chamaedrys

—_ fliformis

- hederifolia

- prostrata

- spicata

- T'eucrium. Mihi.

(V. caulibus adscendentibus, foliis sessilibus cordato-ovatis obtuse serratis, racemis lateralibus longissimis, calycibus quinquepartitis inaequalibus. Mihi .

Veronica Teucrium. Linn.Sp. pl. ed. II. T. I. p. $16 . \mathrm{W}$. Sp. pl. x. 66.

Veronica latifolia. Linn. W. Sp. pl. $\mathbf{x}$. 7 .

Veronica pseudochamaedrys . . Jacq. aust. i. p. 37 . t, 60 .

An Veronica spicata latifolia? Segu. Ver. 1. 234. (non G. Bault. Pin. )

Veronica montana Riv. Nonop. t. 95 .

Chamaedrys spuria major latifolia. G. Bautı. Pin. 248.

Chamaedrys spuria major altera sive frutescens. G. Bault. Pin. 248.

An Veronica supina del LobeIio. Pona Bald. Ital. I69. Segu. Ver. II. 364?

Tencrium quartum. Clus, hist. 349. c. ic.
Radix perennis fibrosa. Caules pedales, sesquipedales, adscendentes, teretes, pubescentes, firmi . Folia opposita, subamplexicaulia, cordato-ovata, obtusiuscula, rugosa, pubescentia, subtus pallicliora, magis minusve profunde obtuseque serrata, quando que angustiora, elliptica, acutiuscula. Racemi ex alis superioribus, oppositi, longissimi, multiflori . Pedicelli bracteis lineari lanceolatis subaequales, pubescentes. Calyx quinquepartitus, laciniis lineari-lanceolatis inaequalibus, duobus nempe minoribus, quinta minima. Corolla coerulea, speciosa. Capsula obcordata, compressa.

In Paldi locis humilioribus et in collium fere omnium sylvulis occurrit ; quamvis a Seguiero cum propriis phrasibus non commemorata; modo major evadens ( $V_{e}$ ronica latifolia) in locis fertilibus, modo minor in siccioribus (Veronica Teucrium).

Ex multis praeclaris Botanicis Veronicam hanc accepi modo sub nomine Veronicae Teucrii, modo Veronicae lalifoliae: eamdemque in provincia Veronensi et proximis collectam ad eos missam modo Veronicam. Teucrium dixere, modo Verouivam latifoliam. Ex quibus colligo unicam esse speciem, cui nomen Veronicae Teucrii serbavi. Meam sententian insignes Willdenowius et Schraderus confirmant, quorum primus in sui operis cui titulus - Species planiarum - 
tom. 1. ץ. 66. sic ait, Specimeu hujus Veronicae (scilicet Leronicae Teucrii) in herbario Linnaeano non invenit clarissimus Smith (Act. Soc. Linn. Lond. r. p. rgr.) Ego semper sub hoc nonine $l^{\prime}$. latifolium, vel $V$. prostratae varietatem majorem vidi, ergo dubia planta " . Et Schraderus in Florae germauicae tom. I. P. 37. "Collatis synonymis a Linnaeo ad! $V$. lutifoliam et $T e u-$ crium relatis, vix ulium dubium mihi superesse videtur, Linnacum sub varietate nostra majori $V$ er. latifolian sub minori $V$. Teucrium intellexisse, quod adhuc respectu $V$. latifoliae magis probabile fit eo, quod in herbario Jacquini fil., ut cl. Schott mihi per litteras mandavit, specimen hujus plautae asservatur, cui Linnaeus manu propria notam ac'scripsit: Veronicam pseu-

Dal luogo suddetto sino I 450 metri crescono oltre Achillaea distans. Acer Pseudo-Platanus.

Aconitum cernuum

- Lycoctonum

Napellus.

Actea spicata.

Agaricus alneus

- coriaceus

- versicolor.

Alchemilla vulgaris.

Apargia alpina

- tuberosa.

Aquilegia vulgaris.

Arabis alpina. (Draba Clusiana di seconda specie. Ponce Bald. ital. 19o. Draba Calceol. viagg. 9.) dechamaediyn Jioq. eandem esse cum sua latifiolia". ) Veronica triphyllos.

Verrucaria ancitepta

- Epipolaca

- Harrimamni

- Schraderi.

Viburnum Lantana.

Vicia angustifolia

- - cracca

- Ervilia

- Intea

- narbonensis

- peregrina

- - sativa.

Vinca minor (Dafnoide. Colceol. Viagg. 9. )

Viola canina

—_ mirabilis

- odorata

- tricolor $\alpha$.

Vitis vinifera.

Xanthium spinosum

- strumarium.

Xerauthemum inapertum.

Xyloma salignum.

Zyzyphus Paliurus

- vulgaris.

all'Ortigara all' altezza di molte delle nomiuate :

Arenaria Gerardi .

A. foliis lineari-subulatis, trinerviis, floribus geminis torminalibus, calycinis foliolis acuminatis marginc menbranaceis trinervits. W. Sp. pl. I I. 729 .

Questa specie trovasi volgarissima iu tulti i pascoli ciella mezzana ąltezza fino alla mas̆giore, e varia perciò senzt modo. Il Seguier (Pl. Veron. I. p. 419) l'à cerlatmente inclicata colla frase "Alsine saxatilis et mulliflora capillaceo folio ". cho spetta all'Aieraria saxatilis, 
cui non ò giammai rinvenuto, tuttochè e' dica essere frequente in monte Baldo massime al Canpedello. Anche nell' Erbario del Mureni trovasi l'Arenaria Gerardi, colla sudetta frase e colla crtazione del Seguier. Nel volume terzo delle Plantie veronenses colla frase Alsine alpina multiflora capillaceo folio flore minore vien descritta una varietà dell' $A$ renaria Gerardi a petali minori, ch' io pure ò raccolto in monte Baldo. Una terza varietà mi venne veduta in copia fra i dirupi di valle Finestra, e per l'abito diverso potrebbe credersi specie distinta. A' questa un caule dritto privo affatto di peli, laddove le altre due sono tutte pelose e viscose, onde la distinguerò coll' aggiunto glabra. Il carattere floribus geminis terminalibus è più costante in questa che nelle due prime varietà.

Arnica montana. Arundo sylvatica. Aspidium fragile. Astrantia major. Astragene alpina.

Atropa Belladona ( Solano maggiore. Pona Bald. ital. 2fo; Calceol. Viagg. 10.)

Avena pratensis.

Betonica alopecuros.

Betula alba.

Boletus fomentarius

- lutescens

- velutinus.

Borrera ciliaris

- furfuracea

— tenella.

Cacalia alpina.

Carlina acaulis
Carlina acaulis ver. caulescens. Carum Carvi .

Cenomyce rangiferina

Centaurea montana.

Cetraria juniperina .

Chaerophyllum hirsutum ß. fl. purpureo.

Cineraria alpina. Mihi.

C. foliis cordatis, inciso-dentatis, petiolis dentatis vel pinnatis, anthodiis paniculato-corymbosis. Mihi .

(C. alpina. Willd. Sp. pl. III. 208\%. Pers. Syn. pl. II. 4 o. Scnecio alpinus. Linn. Suppl. $37 x$.

C. cordifolia auriculata. Jacq. Austr. t. 177.

Cineraria alpina $\beta$. Linn. Sp. p1. 1243.

Jacobaea alpina laciniata flore buphtalmi. Bauh. Fin. I3r. Segu. Ver. III. 278.

Giacobea III. ongarica del Clusio. Pona Bald. ital. $23 \mathrm{r}$.

Jacobaea 3, latifolia I. Clus. Hist. XXIII. c. ig.

- - $\beta$. Cineraria cordifolia . Linn. suppl. 375 . $W$. Sp. pl. III. 2083. Pers. Syn. pl. II. 450. Jacq. austr.t. 176 ,

C. foliis cordatis duplicato dentatis, petiolis auriculatis. Milie.

C. alpina $\propto$. Linn. Sp. pl. 1243. $-\gamma$. Senecio baldensis. Encycl. botan. 7. p. rog. Pers. Syn. pl. II. 437 .

C. foliis petiolatis, ovato-cordatis, inaequalitc: dentatis . Mihi.

Jacobaea alpina foliis subrotundis serratis. Tournef. Instit. 485 .

Jacobaca montana integro rotundo folio. Bocc. Mus. If. p. $6 \mathrm{r}$. t. $49 \cdot$ (inala). 
Conyza alpina. J. Bauh. Hist. II. p. 1055 . c. ic.

Jacobiea alpina foliis rotundis serratis. C. Bauh. Pin. 13̈ı. Prodr. 7o. t. 69. Ponted. Diss. 235. Segu. Ver. III. 27.

Jacobaea latifolia pannonica. Clus. Pann. p. 574. tab. 575. Jacubaea pannonica quarta. Clus. Hist. XXIII.

Ex radice perenni, ramosa canles plures bi-tripedales, herbacei, striato-angulati crecti, superne ramosi in paniculam corymbosam. Folia ornuia petiolata supra fere glabra, subtus villosa vel tomentosa, valde polymorpha, modo cordata, duplicatodentata, petiolis nudis sive uno alterove dente vel auricula foliacea iustructis, modo sublyrata rel pinnata, 1)inna terminali maxima cordata, ambite surrata vel dentata vel inciso-dentata. Corymbi terminales et axillares. Pedunculi tomentosi, bracteis linearibus obsiti. Peranthodium peranthodiolatum, bracteis subtomentosis linearibus, apice saepe sphacelatis. Corollulac Inteac. In pratis fertilibus praesertim circa pastorum caulas nimis frequens occurrit in baldo, et in omnibus montibus $\mathrm{Ve}$ ronensibus, Vicentiuis, Brixiensibus, et Rheticis. Quintili et Sextili mensibus floret. Nel fascicolo pubblicato delle piante nuove o poco conosciute del Veronese ò fatto veduto, come la Cineraria alpina e la Cineraria cordifolia di Linueo, Willdenow, e Persoon sieno una sola specie, cui ì lasciato il no- me dii Cincraria alpina. Ora agsiunso, che it Senecio liuldensis dal Bose rinvenuto in monte Baldo, e descritlo dill'Enciclopedia e dal Persoon, ¿̀ senza dubbio varielà della mia Cineraria alpina. Imperocchè ò osscrvato piì volte in monte Baldo sorgere dalla stessit radice individui, che aveano i caratteri delle tre varietid accennate. Oltraccio avenelole io per varj anni coltivate nolI'Orto botanico di Verona, le ò vedute trasformarsi a vicenda le une nelle altre). Collema flaceidum.

Convallaria verticillata.

Corydalis bulbosa

_- lutea .

Cytisus alpinus

- Laburuum.

Dacdaleat quercina .

Daphne Mezereum .

Digitalis ambigua.

Epilobium angustifoliım .

Euphorbia dulcis

- sylvatica.

Evernia prunastri.

Fagus sylvatica.

Galeoludolon luteun.

Galium austriacum

- sylaticum .

Gentiana acaulis

- asclepiadea cruciata

- - G. G. cruciata minoa

$$
\text { germanica }
$$

verna.

Geraninm pliocum

- pratense.

Geum rivale.

Ginaphalium dioicum a fl. [mpureo ß. fl. albo

- sylvaticum.

Hierarium integrifolinm. 
Hyosceris fuetida.

Hypericum montanum .

Jungermannia complanata .

Lecanora atra

- salicina

- subfusca

- varia.

Lecidea parasema.

Lepraria chlorina.

Lilium bulbiferum

- Martagou.

I.onicera alpigena

Luzula maxima

- nivea

- pilosa

- spicata.

Lychnis sylvestris.

Mielica coerulea.

Melissa pyrenaica.

Irilium effusum.

Mnium undulatum. Hedr.

Nepeta nuda.

Orchis conopsea.(Segu. Ver.II.

p. 124. n. 5. et III. p. 251.

n. 9. tab. 8. f. 7 .

- Morio.

Ornithogalum pyrenaicum.

Paeonia corallina

- officinalis.

Tarmelia glomulifera

- olivacea

- pulverulenta.

Peltidea horizontalis .

Phleum alpinum.

Plantago montana. Lamark. Por alpina

Polygala $\mathrm{C}$. vivipara.

Polygonum Bistorta.

- viviparum.

Potentilla aurea.

Primula veris.

Pyrus Aria

- torminalis.

Ramalina farinacea

- fastigiata

- - $\beta$. calicaris

- fraxinea
Ramalina pollinaria.

Ranunculus aconitifolius.

Rubus idaeus

—- saxatilis .

Rumex alpinus.

Salix Wulfeniana .

Salvia glutinosa .

Sanicula europaea.

Saponaria ocymoides .

Saxifraga rotundifolia.

Scabiosa sylvatica .

Scrophularia verna .

Sedum saxatile?

Senecio laciniatus. Bert. Journ. bot. 4. p. 76. S. foliis imis petiolatis ovatis, inciso-dentatis, caulinis auriculato-amplexicaulibus, remote pinnatifidis, laciniis oblongis dentatis; peranthodiis glabris; seminibus striatis, piIosiusculis . Mihi.

Soldanella montana.

Solorina saccata.

Sorbus Aucuparia.

Spallanzania Agrimonoides : foliis radicalibus pinnatis, caulinis ternatis, impari majorc, basi cuneiformi. Pollin. Pl. nov. vel min. cogn. pag. 10. fig. I. A B. C. D. E. F. G. Al Pra-lungo.

Carattere generico

Calyx duplex campanulatus roI 2-fidus, internus 5-fidus fauce contractus. Corolla quinquepetala . Stamina octo summo calyci affixa. Orarium unicum, styli duo, stygmata duo. Semen unicum, globosum, calyce interno capsulari inclusum .

Appoggiato all'autorità dei celebri Anton Lorenzo Jussieu e Ventenat, non meno che al consiglio dal mio chiarissimo amico Balbi, ò istituito nel citato mio fascic olo 
roll' Agrimnnia Ayrimonoides il genere Spallanzania in onore dell' immortale mio maestro. E la ragione che m'indusse a ciò fare fu la souma discrepanza nel carattere generico, e principalmente pel calice interno non coperto nel mezzo di sete uncinate, per l'esterno non minimo né bipartito, ma di uguale grandezza dell' interno, e 10-12-fesso, pel numero degli stami, clie sono otto anzi che dodici a venti, e infine per l'ovario unico e non doppio, e per la casella monosperma non setosa. A tutto cio vuolsi aggiungere l'abito della pianta, la quale è fornita di foglic cauline non pennate, ma ternate.

Spiraca Aruncus.

Sticta pulmonacea - - sylvatica.

Stilbospora microsperma.

'Teucrium Botrys.

'Thalictrum aquilegifolium .

Thclephora hirsuta
Thelephora purpurea

- quercina.

Thesium Linophyllum.

'Thlaspi arvense

Thymus alpinus. (Clinopodio anstriaco del Clusio. Pona Bald. ital. 2ro. 2qr.)

Tormentilla erecta.

Trollius europacus.

Turritis glabra.

'Tussilago alba

Uredo gentianac. Stranss. In foliis Gentianae oruciatae.

Unica dioica.

Usnca harbata

- - - $\beta$. dasopoga

florida

- plicata.

Valcriana montana

_- officinalis

-. tripteris.

Variolaria communis

- - $\beta$. faginea .

Verbascum nigrum.

Veronica officinalis

- serpillifolia

- urticacfolia.

Vicia sepium.

\section{Dall'Ortigara si ascende in Costa-bella, dai 1450 a}

\section{0 metri .}

Alchemilla alpiua ( Tormentilla caudida del Dalecampio. Pona Bald.ital. 188. 195.) Apargia alpina.

Achillua Clavennac.

Arenaria ciliata. (Alsine serpilli folio inulticaulis et multiflora. Segu. Ver. I. p 420. tab. 5. fig. 2.)

Arnica Bellidiastrum.

Aster alpinus.

Astragalus inontanus.

Carex ferruginea. firma
Carex mucronata

_- ovalis.

Cetraria islandica.

Cherleria sedoides. (Segu. Ver. III. p. 180. tav. 4. fig. 3.)

Dapline Cncorum .

Draba aizoides.

Jryas octopetala.

Erygcron alpinum.

Galium baldense. Caule adscendente, foliis senis crassiusrulis glahris acutiusculis, pedumculis axillaribus subtrilluris, fructibus glabris. Spec. 
nova alio in loco descrip'a .

Creraium argenteum .

Geum montanun.

Globularia cordifolia .

Gruphalium Leontopodium.

Helianthemum grandifiorum . $D_{e} C$. ( Helianthemum alpinum vulgari simile, latioribus foliis. Se gu. Ver. III. p. r $9^{3}$. t. 6.f. s. )

Helianthemum oelandicum . (H. suffruticosum procumbens extipulatum, foliis. oppositis ovato-oblongis subpubescentibus, calycibus villosis. Arihi.

Cistus oelandicus Linn. Sp. pl. Helianthemum alpinum, serpillifolio nigricante et hirsuto. Segru. Ver. III. 195. t. 6. f. 2 .

-_ - 3 . Cistus serpillifolius. Linn. Sp. pl.

H. suffruticosum extipulatum procumbens folis oppositis oblongis pubescentibus, calycibns villosis. Mithi.

H. serpilli folio, flore majore aurco, odorato. Segu. Ver. III. p. 19' . Moreni et Bordon. lort. sicc.)

Uua leggiadra specie che non trovo descritta nè dal Linneo, nè da tutti gli altri cditori delle sue Spexies plantarum ì guella che il Seguier (11. Ver. III. p. 195.) à determinato colla seguente

- frase, Heliantlicmum Serpilli folio, flore amp̧lo, sulphureo ", c di cui ì arauti agli occhi due esemplari negii erbarj del Moreui e del Jordoni. Io ia chiamerò

Helianthemim Seguieri.H. snffruticosum prccumbens, stipulatum, stipnlis linearibus acutis, foliis sessilibus opposilis ovatis subpulescentibus, sepalis ovatis nervosis, subpilosis. Mitui.

Proximum Helianthemo oclandico, sed differt caule stipulato, sepalis longe amplioribus, usumquodque 3-/4-nervis nigris pilosis exaratnm . Corolla nuagna Helianthemi grandiflori sed pallidior.

Hieracium integrifolium.

Lecidea saxetana.

Ophris alpina.

Orchis nigra ( Segu. Ver. II. p. 133. n. 25. tab. 15. f. 17.) —— viridis (Segru. Ver. II. p. 133. n. 26 . t. 15 . fig. 18. et (a]). 16. fig. 18.)

Potentilla aurea.

Ranusculus alpestris. ( Ranunculus alpinus humilis, albus, folio subrotundo. Serru. Ver. I. 489 . tab. 12. ig. 1.)

\section{- montanus}

rutaefolius ( Ranunculo alpino con foglia di Coriandro. Pona Bald. ital. 446. c. fig. )

Saxifraga aizon a major longifolia.Sternb.S. recla.Lapeyr.

_- _ E. minor brevifolia. Sternb.

( Saxifraga sedi folio angustiore serrato. Segu. Ver. I. p. 4is. t. 9. f. x.)

- cocsia ( Saxifraga alpina minima, foliis coesiis, deorsum recurvis. Segu. Ver. I. tab. 9. fig. 2.)

Silcne acaulis (Ocimoide muscoso alpino. Ponce Bald. ital. 199. c. fig. )

Spergula saginoides.

Tofieldia palustris. ( Phalangium alpinum palustre, iridis folio. Segu. Ver. II. pas: 6r. tab. 14 Falsoasfodelo $\mathrm{il}$ Clusio. Pona Bald 1;4.271.) 
Urceolaria cincrea $\delta$. U. Lessulatia.

Valeriana saxatilis.

( "ino dai primi anui che presi a scorrere erborizzando il monte Baldo e i prossimi monti Veronesi, Tirolesi, c Vicentiui mi nacrue sospet1n, che Calceolari, Poua, e Seguier avessero scambiata Ja Valeriuna suratilis colla I'aleriana celtica. In.perocrhi la Valeriuna suxatilis, ch'io rimvenia volgarissina in tutle le nostre Alpi, lai citati autori nou fa osservata, c il Seguier la descrive sulla fede di Gasparo bauTruo, che in Balda la trorò. All'opposto per quanla sollecitudine io abbia usato non sono giunto a scoprire un solo esemplare di $/$ aleriana celeica, ad ontit che Calceolari la rinvenisse nella valle dcll' Artillon, e Pona frequentissima nei dirupi di valle-fredda, e al dire del Sesuier cresca in copia sulle cime di Baldo e degli altri nostri monti. L'esame clie instituii poscia denli crbars del Moreni e del liordo:ia, ambeduc colleghi nei riaggi e amice del seguier mi volsero il sospelto in certerzat. Imperocelic is veduto colle diasi . Nurdus alpina. Clus. hist. LVII. c. ir. Nardus celiica.J. Baul. II ist III. 205 . c. ic. Valeriana celtica. Tumrnef. Instit. 13 I, tuna varicta a foglie interissime della Valeriana sassatile da me pure sovente veduta, per veriti assai simile alle ligure del Clusio e di Giovami Bunhino. Che i suddell antori abhiano realmente preso la V. sassatile per la V. celliea me lo conferma il Wulfen, il quale asscrisce, che la V. coltica suole crescere solo nei monti di granito, gneisso, e di simili rocce primitive, e i nostri monti come è noto sono secondarj).

Veronica apliylla (Veronica parva sasatilis caulieulis nudis. Segu. Ver. I. p. 2,y. tab. 3. lig. 2. et Vol. III. p. 116.$)$

Nella valle Vaccaria e al fonte di Brigallello, dai

\section{I700 ai I900 metri.}

Angelica sylvestris $\beta$. A.montana fuliis elliptico-lanceolatis.

Anthyllis montana.

Atragene alpiua (Clematide cruciata alpina. Pona Pald. ital. e fig. )

Carex capillaris (Cyperoides alpinum, spicis senuiniferis pendulis, binis in sunmo caulc. Segue. Ver. III. p. 83. tab. 3. f. 1.)

Gentiana lutea.
Juniperus nana.

Laserpitium latifolium

-_ pencedanoides. (Laserpitium pencedanoides, foliorum setrmentis angustissim is. Segur. Ver. III. p. 227 . t. 7 . An Peuccdanum. Calc. Viagg. If?

Oplirys alpina.

Orrhis odoratissina Sesu. V. IH. p. 250. 11. 8.18 i.6.

Pedicularis commsat.

Phytema orbicularis 
Phyteuma spicala.

Pinus Pumilio.

Polygala Chamaebuxus var. $f$. alis purpureis, carina apice flava.

Jrimula veris.

Saxifraga androsacea.
Saxifraga Pona . . . Sternb. (Saxifraga alba petraea. Segu. Ver. I. 447. Pona Bald. ital. r83.c. fig. buona. Sassifraga bianca. Calc. Viagg. 12.)

- _ ${ }_{-}$. Sternb. foliis omnibus integris.

Nella valle alpina detta di monte maggiore, o valle delle pietre, e lungo il dirupato sentiero chiamato scalette, onde si ascende all'alta vetta, e nei pascoli prossimi al vertice, dai r800 ai 2200 metri.

Ajuga piramidalis .

Athamanta cretensis.

Azalea procumbens.

Bartsia alpina.

Chaerophyllum sylvestre.

Cherleria sedoides.

Dianthus sylvestris.

Galium baldense.

Marchantia hemisphacrica

- polymorpha.

Paederota Bonarotta: foliis subrotundo-ovatis dentatis; racemo oblongo subnt:tante, corollarum galea indivisa acuta. Pollin. Element. botan. tom. II. p. 153 . tav. 5. fig. 2. (Paederota Bonarotta et Veronica Ponae. Willd. Sp. pl. et Pers. Syn. pl.

Veronica Bonarotta. Linn. Sp. pl.

Veronica petrea. Segu. Ver.I. p. 235. Pona Bald. ital. 179. c. fig. e 244 .)

Pedicularis rostrata

- tuberosa.

Pinus Pumilio.

Potentilla nitida.

Primula Auricula

- carniolica.

Ranunculus alpestris

- a uricomus: foliis radicalibus reniformibus crenatis, caulinis sessilibus digitatis, linearibus serratis. Milii.
(R. rotundifolius vernus sylvaticus. Bauh. Hist. III. 857 . c. ic.)

Ranunculus lanuginosus

- montanus

- rutaefolius

- Thora

Salix herbacea

—_ reticulata

- retusa.

Saponaria ocymoides .

Scorzonera alpina. Milie.

(S.scapo monanthodio aphyllo simplici, foliis lineari-acuminatis, glabris, nervosis, planis. Mithi.

An Scorzonorae austriacae W. Sp. pl. varietas?

Scorzonera angustifulia prima. Moreni hort. sicc.

Ex radice perenni superne squamis nigris olotccta, scapus teres, glaber, pedalis et ultra, simplex, monanthodius, superne quandoque unibracteatus. Folia fere pedalia, lineam circiter lata, non rigida, integerrima, linearia, longissime acuminata, glabra plana 3-raro 5-nervia, nervo medio interdum albo. Peranthodium glabrum hasi subtomentosum : bracteis peranthodialibus lanceolato-a- 
cuminatis, margine albo memJranaceo. Corollulac peranthodio duplo longiores lineares quinquenervosae truncato-quinquedentatae, una cum stylis luteae.

Mihi florens occurrit Quintili et Sextili mensibus in editissimis Baldi jugis; primum in pascuis di monte magrgio$r e$, adscendens ex valle ejusdem nominis ad cacumen, dein'in valle delle os$s \pi$, demumque in pascuis summi jugi vallis Finestra. Morenius quoque in Baldo monte decerpsit, et cum phrase Bauhini pinacis pracdicta in proprio herbario de- (erminavit).

Silene acaulis

- - - B. fl. alloo

-m Saxifraga (Lychuis minor, Saxifraga. Segu. Ver.I. 431. tah. 6. f. 1. Sassifracia maggiore degl'Italiat. Pona Bald. ital. 176. Sassifragia maggiore del Mattioli. Calceol. Viagg. 9. )

Trifolium alpinum. ( Trifoglio angustifolio alpino. Pona Bald. ital. 19亿. c. fig.)

Tussilago discolor.

Valeriana montana saxatilis .

Veratrum album

- nigrum.

\section{Da Costa-bella proseguendo pel Coval santo, Mon-} maor, Sascaga, e per le seguenti altissime vette fino oltre la Colma o cima di valle Finestra, dai 2000 ai 2200 e più metri.

\section{Achillea atrata}

\section{Clavennae.}

Alchemilla alpina.

Androsace alpina. Lam. Aretia alpina. Linn. W. Sp. pl.

\section{- lactea.}

Anemone alpina.

Apargia crocea. Sotto il Corcel santo.

(A. scapo monanthodio, superna parte squamosa peranthodiisqne hirtis, foliis runcinatis glabris, lacinia terminali triangulari. Pollin. Pl. nov. vel min. cogn. p. r9.) Arabis nutans

- saxatilis.

Arenaria ciliata .

Astragalus montanus.

Athamanta cretensis.

Bartsia alpina. (Clinopodio alpino. Pona Bald. ital. 20 - .c. fig.) Bupleurum graminifolium ( Se- do petreo con foglia di Buplenro. Pona Bald. ital. $2 q^{\prime j}$. c. fig. )

Carex alpestris

- capillaris

- ferruginea

- firma

- mueronatia

- ovalis. (Carcx angustifo-

lia caule triquetro, spicis pluribus elegantilus parum inter se distantibus. Segu. Ver. I. p. 124. t. 1. f. 3.)

Cenomyce vermicularis. $\beta$ taurica.

Dianthus plumosus. $D_{C} C$.

Draba aizoides

- pyrenaica. In Monmaor. Dryas octopetala.

Evernia divaricata. In Rileododendro Chamaecysto

Geranium argentcum. (Geranium argentcum alpinum, 
Inngius radicatum. Segru. Ver. I. p. 471. tab, 10. Geranio apino. Pona Bald. ital. 2or. c. fig.

Ceum montanum (Caryophylata alpina lutea. Segic. Ver. I. 49'. Cariofillata montana del Matlioli Pona Bald. ital. 189. 214. 236. Culc. Viagg. 1年.)

- - B. (Ciryophyllita alpina minor. Segre. I. 49 J. Ciariofllata alpina minima di prima specie. Pona Bald. ital. 203. c. fig. )

Fileracium aureum

- _ chondrilloides

- cymosum

- villosum.

Juncus trifidus. Nei pascoli di Monmaor .

Iberis rotundifolia.

Lepidium alpinum

- petraeum.

Jycopodium selaginoides.

Nardus stricta.

Pedicularis rostrata

- - - var.fl. carneo. Mon-

maor.

- tuberosa.

l'hyteuma comosa. Coval sanLo. (Trachelio minore pe1reo. Pona Bald. ital. I77. c. fig. e 2 年.
Pinguicula flavescens. Flork.

Schrader.

Polentilla nitida. Coval sunto, Alonmaor.

Primula Auricula

- carniolica.

Ranuvculus alpestris. (Ranunculus alpinas humilis, albus folio subrotundo. Segu. Ver.

I. p. 489. tab. 12. f. I.)

Rhododendron Chamuecystus. Monmaor, Sassetto.

Salyx myrsinites

- - reticulata

- retusa.

Saxilraga bryoides

- coesia.

-_ exilis. Mrihi. Coval santo.

( $S$. caule subunifloro foliis alternis, lineari-lanceolatis, radicalibus in rosulam dispositis, spathulatis. Pollin.

Pl. nov. vel min. cogn. p. II. - - muscoides.

Trifolium alpinum. Monmaor. ('Trifolio angustifolio alpino.Pona Bald.ital. 19 4. c. fig.) Tussilago discolor.

Urceolaria ocellata.

Valeriana saxatilis.

Viola biflora ( Viola gialla del Clusio. Pona Bald. ital. p. 237. Viola gialla . Calceol. Viagg. 12. )

Nelle valli alpine dette delle $O_{s s a}$, di $S$ Zeno, valle grande o dritta, valle Orzera, e valle della Finestra, dai 1700 ai 2200 metri .

Acliillea atrata .

Alyssum rupestre W. Euum. 672. ('Tlaspi alpino petreo con aspetto di Miagro. Pona Bald. itul. 185. c. fig.)

Anemone baldensis. Valle delle ossa. (A. foliis biternatis, foliolis tripartitis, laciniis trifidis, involucro folioso, seminibus lanatis stylo persistenti brevissimo. Willd. Sp. pl. II. 1 278.)

Anthemis alpina.

Arbutus alpina. Valle grande. Arenaria Greardi $\alpha$.caule jilosa viscoso. 
Arenaria Gerareli $\beta$. pelalis calyce minorilus vel subacqualibus. Valle granace.

nestra.

- polygonoides. Valle grande. (Segu. Ver. III 17\%. t. f. f. 1.)

striata. Valle S. Zeno, valle grunde.

Arnoria vulgaris. Scapus glaher, hracteac peranthodiciles csteriores acutae.

Arnica Be!lidiactrum.

- scorpioides: foliis dentatis, radiralilius longe petiolatis subrordito-ohlongis, caulinis alternis oblongis, summis amplexicaulibus cordato-lanceolatis. Pollin. Pl. nov, vel min. cogn. p. 3 j.

V 3 . A. glacialis. W garico primo del Clusio. Puna Bald. ital. 2qu.)

A. Wulfeniana Pollin. A. cordata. Wulf: Valle di S.Zeno, valle $I i-$ nestra.

Nel citato mio fascicolo delle piante nuove o meno conosciute Veronesi a c. 35 . 36. ì fatto veduto, che l'Arnica scorpioides e l'Ainica glacialis erano varietà della stessa specie nata in plaga piu o meno alpestre. Descrivendo poscia l' Ainica cordata del WVulfen, cui ò mutato il nome in Arnica $W_{\text {ul }}$ feniana, perchè esiste già altra Arnica cordate descritta dal T'hunberg, o aggiunto, ch' io ammctucra ta!e specie appoggiato ali' autoriti clei cẹlebri Sternberg, WVulfe:ı, Persoon, ma che inclinava a crederla una varicta del-
l'Arnica sunipinides. Nell'ultima gita fatta nell Agosto dell anno corrente arendo raceolto varj esemplari d'Arnica seorpioides e drnice II ulfenirana specialusente in valle Finestra, ove fu veduta anclie dallo Siernberg, nii sono arvedulo del graduato passaggio dell'una nell'altra specie; e ò pure coufermato insussistente il rarattere del seme nudo e del perantoclio bislungo accordato dillo Steruberg all'Arnica Wulfeniana. In tumi s!i csemplari da me raccolti il seine era provreduto di filppo, e il perantodion non dissimile da quello dell' Arnica scorpioidle.

Aspidium alpioum ( Filicula alpina crispa. Segu. Ver. III. 1. 55 tab. x. fis. 3.bona. et I. tab. r. fig.r. mala. Felce crespoo sassatile. Pona Bald. ital. 2z. (. fig.) - fragile

- - Aspridium Pontederac. W. Valle grande. (L'Aspidium Pontederac VV.o Polypodium Pontederae delJ'Allioni, rinvenuto dal Pontedera per il primo nei luoghi alpestri di monte Baldo e figuraio dal Segnier ( PI. Ver. III. tab. I. fig. 2. ) \& certamente una varietà della specic fuor di modo polimorfa Aspidium fiagile. Io ne ò trasportato dalle valli srande, e Losanna più piedi nell' Orto botanico di Verona, e in due o tre anni di coltirazione andarono convertendosi in Aspidium fiagile. Nello stesso monte Baldo ì colto esemplari, che aveano 
i caratteri d'ambedue le varictà, costituendone il passaggio ).

Aspidium rigidum (Filix non ramosa, alpina foliolis, quac ad alas rotundioribus omnibus autem dentatis. Ponterd. Comp. I3. Segu. Ver. III. p. 53.tab. r. fig. r.)

Athamanta cretensis .

Cerastium latifolium.

Geranium macrorıhizon. Valle delle ossa.

Heracleum pyrenaicum . Volgare fra $i$ dirupi della valle delle ossa.

(H. foliis simplicibus subtus incanis, rotundo-cordatis, trilobatis, lobis lobulato-angulatis, dentatis. Pollin. M. nov. vel min. cogn. p. 8.

Sphondylium alpinum parrum, et Sphondylium alpinum glabrum. Segu. Ver. III. \$25. Moreni hort. sicc.

Questo Heracleum da me descritto nel predetto fascicolo a c. 8 . 9. non pare l'Heracleum pyienaicum della Flora Taurico-caucasica del Bieberstein (Vol. I. p. 224.) e riferito dal Willdenow nella sua Fnumeratio a c. 3 I 2 : imperocchè il seme del mio Eracleo pirenaico à il margine interissimo liscio, e non cigliato-scabro. Mi è nato dubbio, potesse esscre l'Ileracleum gummiferum descritto dal Willdenow a c. 53 . e figurato nelle tav. 53. 54 del1'Horlus Berolinensis, e cui asserisce d'aver ottcuuto dai semi tratti dalla Gomma anmoniaco del commercio : sicchè pensa esnere un tale Eracleo la pianta d'onde si estrae la Gomma sucldetta .
Di fatli tutta la pianta dell'Eracleo di monte Baldo à un odore grave assai simile a quello della recente Gomnia ammoniaco, e di tale una veemenza, che uom non dura a fiutarla lungameute. Però le foglie non sono per di sotto pubescenti, nè la pianta c bienne, ma perenne).

Iberis rotundifolia.

Juniperus naria.

Lepidiun alpinum .

Linaria alpina (Linaria quadrifolia supina. Segu. Ver.I. pag. 265.)

Lychnis quadridentata . Valli grande e orzera (Lychnis alpina foliis angustis reflexis petalis quadripartitis. Segu. Ver. III. p. 186. tab. 5. f. 1.) Mespilus Cliamae-Mespilus.

Pacderota Bonarotta.

Papaver aurantiacum . Lois. Notic. 84.

(P. scapo foliisque pinnatis hirsutis, pinnis ellipticis subdentatis vel pinnatifidis, capsulis ovatis hirsutis. Milii. Papaver alpinum. Sternb. Reise in die Khet. alp. 5.r.

Papaver alpinum, saxatile, Coriandri folio. Segu. Ver. I. 416. tab. 4. fig. 4.

Argemonc alpina, Coriandri folio. C. Buul. Pin. 172 . Argemone giallo. Pona Bald. ital. roo. c. fig. catt.

Ex radice perenui scapi r-plures spithamei erecti $\mathrm{vel}$ adscendeutes hirsuti uniflori. Folia omnia radicalia petiolata conferta, hirsuta, pinnata, pinnis ellipticis deutatis vel pinnatifidis, raro integerrimis. Sepala 'hirsuta. Petala amplat, aurantiaca 
olorata. Capsula ovata hispida, stigmate 5-6-radiato. In vallibus delle pietre, srande, Finestra, Quintili it Sestili mensibus floret. Provenit etiam in montibus $\mathrm{T}_{\mathrm{y}}$ rolis, et circa Feltrian in jugis diclis le velle.

A Pupazero alpino, cum quo commutatum fuit, differt foliis hirsutis non glabris nee bipinnatis, petalis nom aibis vel pallide luteolis basi flavis, sed ex toto aturatiatcis.)

Phy tcuma comosa. Valli delle ossa, S. Zeno.

Pinus Pumilio.

Potentilla caulescens

- vitida. Valle di S. Zeno. ('Trifolio argentalo alpiun. Pona Bald. ital. 202. c. fig.) - - var. 1 ll allo. Ivi.

Rauunculus alpestris

_- auricomus

- rutacfolius. (Panunculus rutaceo folio, five suaverubente. Segu. Ver. I. fso. Ranunculo alpino con foslia di Coriandro. Pona Biald. ilal. 197. c. fig.)

- Seguieri. Valli delle ossa, S. Zeno. ( Ramuurulus alpinus apii folio, flore alloo inagno. Punted. Comp. 1 Segu. Ver. I. 99o. tab. 12. (ig. 2. 3. folia).

\section{- 'Thora}

Phamnus punilus.

Rhododendron Chamaecintus

- ferrugincum

- hirsulum.

Fiosa alpina.

Salix ambigua

- corruscans

- hedhacea

- my rsinites
Salix reticulata

_- Wulfeniana.

Saxifraga aizoides. Stemb.

- Aizoon B. Sternb.

- androsac ea. Valle grande.

- Iryoides. Valle grande. burseriana. Valle lineslia.

(Saxifraga alpina, foliis glatacis acutis, nonaulios, can-

le fulioso. Segn. Ver. III.

l. 201. A.1. 5. fig. 2. )

- - roesia

_- museoides. ( Sissifiara

pyrenaica, fuliis palim inlegris, partim tritidis. Segue.

Ver. I. 1'. 行 tab. 9. fig. 4.

ct III. P. $20 j$.

- mutala. Valle grande.

- Jonic $\alpha$. Sternú.

___ sedoides. ( Saxifraga alpina muscoides, fuliis silperioribus oblongis, inferioribus rotundiorilus et circumartis. Segu. Ver. III. 203. lab. 3. fig. 3.bona: ec Saxiliraga alpina minima, foliis ligulatis, in orbem circunatctis, flore ochroleuco. sesite. Ver. I. 4́̃o. t, 9. tig. 3. jessima).

Silere acaulis

- Saxifraga.

Thalictrum miusus. Valle delle ossu. ( 'T. alpium minus sasatile Rutac fulio, staminibus lutcis. Tita. it. alp. 19. Segu. Ver. I. $4=6$. titl. 11.

Thymus alpinus.

T'ussiliggo alloa

- discolor

- nivea.

Vaccinium Mrrtillus

- Vitis Idca. Valle grande.

Valeriana saxatilis. 
Ai pascoli e selve dette dei Zocchi e Tredespin, e alla $C_{n} l-$ ma e al pra di Malcesine, dai 1400 ai 1600 metri. Apargia Taraxaci.

dietonica alopecuros

- hirsuta.

Bupleurum ranunculoides.

Campanula bononiensis. Treclespin .

\section{- - barbata.}

Carex alba

\section{- flava.}

Centaurea phrygia. Prato di Malcesine.

Cnicus rivularis. ( C. foliis sessilibus oblongo - cllipticis, glabris subtus pallidioribus, sinuatis, laciniis ovatis, remote scratis, spinulosis, superioribus laciniatis; anthodiis glomeratis, baacteis peranthodialibus lauceolatis, mucronulatis patentibus. Mihi.

Radix porennis. Caulis fere orgyalis ramosus erectus, striatus, glaber. Folia radicalia subsessilia, glabra, subtus pallidiora vel glaucescentia, pedalia vel sesıjuipedalia, oblongo-elliptica, acuta, sinuata, laciniis ovatis acutis, late serratis, serraturis ciliato. spinulosis; cauliua in auriculam rotundatam spiunlosam amplexicaulem utrinque producta; summa Jaciniatopinnatifida integerrima. Peduaculi 2--3-bracteis amplcxicaulibus cordato-lanceolatis, spinuloso-dentatis instructi, quarum una sacpe sub anthodio, superne pubescentes, 2-3-4-raro5-anthodii . Peranthodium hemisphocricum, bracteis lanceolat is mucronulatis patentibus vel paululum reflexis. Corollulae purpureac. Stylus simplex.

Semen ellipticum, compresso-tetragonum. Pappus plunosus.

Mihi occurrit in declivitatibus umbrosis dei Zocchi, tum et in sylvis editioribus quae sunt in descensu ex la bocca di Navene ad lacum benacum. Quintili et Sextili mensibus floret.

Addidi definitionem et descriptionem hujus speciei, quam C. rivularem dixi ex comparatioue speciminum a celeberrimo Botauico, amicoque Balbio acceplorum, quia definitiones Murrayi, Jacquini, et Willdenowii illi minus conveniunt).

Cytisus alpiuus.

Geranium phocum

- pratense

- rotundifolium.

Helleborus niger.

Hyppochoeris helvetica. Colma di Malcesine.

Laserpitium Siler.

Linum hirsutum .

Pedicularis acaulis. Colma di Malcesine .

Rumex Acetosa f.

Selinum Seguicri.

Silene nutaus.

Stachys alpina.

Veronica saxatilis. Ai Zacchi. ( Veronica alpina con foglia di Serpillo. Pona Bald. ital. 13r. c. fig. )

Veratrum album. Ivi.(Elleboro bianco di for pallido. Pona Bald. ital. 169. Elleboro bianco. Calc. Viagg. 12.) - nigrum. (Elleboro bianco con fiori, che nel purpureo nereggiano. Pona Bald. ital. I70.) 
Nella selva di Malcesine, 1200. I300 metri circa.

Alnus incana.

Asarum europacum.

Betula alba

- ovita.

Boletus foinentarius

- rersicolor.

Borrera ciliaris

furfuracea .

Cenomyce furcata $\gamma$ erpemena

- - - $\delta$ recurva

- - ל pungens

- - o spitiosa.

Cetraria islanrifica

- - juniperina $\beta$. pinastri.

Evonimus latifolius.

Giaphis scripta.

Lecanora alliella.

- varia e apocliroca.

Lecidea corticula $\beta$. Ieucocelis. Mespylus Chamae-Mespilus Cotonenster.

Parmelia aipolia

_- aleurites

- caperata

- parietina

Lungo i dirupi del sentier Alyssum rupestre.

Angelica sylvestris $\beta$. montana. Authemis alpina.

Arbutus alpina

_- Uva ursi.

Arenaria ciliata

- bavarica

- striata.

Athamanta crelensis.

Atragene alpina .

Carex Drymeja.

Cenomyce coccifera

- cornuta

— deformis

C- pyxidatia.

Cetraria cucculata $\gamma$. tapeina .

Cnicus erisitales fl. Iutcolo

- - - fl. purpureo

- palustris. Presso la fonte dell' Albio.
Parmelia saxatilis

_- stcllaris

- - tiliacear .

Pinus Abies.

Ossern. Il Pinus baldensis di

Atulio Yucragni Centur. I.

Oloserv. botan. in Collect.

Roemeri pag. 158.) ¿̀ una varictì di lievissimo inomento del Pinus albies.

Pinus Larix

- pirca

- - sylvestris.

l'yrola unillora

-- rotundifolia

- - secunda.

Sticte pulmonacea

- - sylvatica.

'Taxus baccata.

Thelephora quercina .

Usnea barbąlat

- - B. dasopoga

- ceratina

- florida

- plicata.

di Ventrar, I7oo metri. Collema flaccidum.

Convallaria bifolia

- verticillatia.

Cypripedium Calcolus. (Calceolus. Serg. Ver. III. par. 2.).3. Damasonio bastardo de? moderni . Pona Bald. ital. p. 239.$)$

Dicranum adianthodes.

Eirthronium Dens canis.

Gentiana asclepiadea.

Hieracium iucanum

- villosum.

Jungermanuia pubescens .

Iychuis quadridentata .

Pitederota Bonarotta.

Peitidea aphtosa

- horizontalis.

Potentilla caulescens.

Prenanthes muralis 
Prenanthes purpurea

- - - var. fol. angustiojibus.

Rhododendron ferrugineum

- hirsutum.

Rosa alpina.

Sambucus racemosa.

Saxifraga longifolia .Lapeyr . (S. lişulata. Bellard. )

Saxifraga mutata

- Ponae $\alpha$.

Silene Saxifraga .

Tussilago nivea .

Vaccinium Myrtillus

- Vitis Idaea.

Alla Bocca di Navene e nelle prossime selve lungo la via che scorge al Lago, dai 1300 ai 1600 metri. Aecidium cornutum. Asplenium viride. Bartramia Oederi —— pomiformis.

Cenomyce rangiferina $\beta$. syl- Laserpithium prutenicum. Nelvatica

- - $\gamma$. alpestris

- bacillaris

- - $\gamma$. macilenta.
Cnicus rivularis.

Jungermannia Erhartiana. $W e b$.

Nelle selve lungo la discesa . -

le selve inferiori.

Mercurialis perennis.

Rubus hirtus.

Sclinum Seguicri.

Al luogo detto il Lastè oltre Navene, ed in Altissimo, dai 1600 ad oltre 2100 metri.

Amanita bulbosa. In Campo presso l'Altissimo.

Aira montana.

Barbula inclinata. Schwaegrig. In Altissimo .

Cenomyce vermicularis $\beta$. taurica.

Centaurea Rapuntica.

Cetraria cucullata $\beta$. nipharga. Cynontodium capillaceum . Hedw.

Geum montanum .

Jungermannia resupinata. $A l$ tissimo .

Nardus stricta. Volgare ne'pascoli dell' Allissimo .

Orchis globosa (Segu. Ver. II. p. 129. n. 17. tab. 15. f. 12.)

Dalle falde settentrionali verso Tierno ${ }^{\circ}$, Castione, Brentonico, valle delle Sorne ascendendo per S. Giacomo', Pozzaferrera fin presso ai Pianetti; dai 300 a incirca 1000 metri.

Orchis nigra viridis .

Pedicularis tuberosa

- vertícillata. Altissimo.

Salix herbacea. In Campo .

Saxifraga aizoides

- coesia

—- sedoides.

Senecio Doronicum $\beta$.

Spartium radiatum .

Spergula saginoides. Nei pascoli. S. caulibus simplicibus decumbentibus, foliis oppositis lineari-subulatis, pedunculis axillaribus, petalis calyce brevioribus. Pollin. Pl. nov. vel min. cogn. p. 12. verso Tierno, Castione,
prne ascendendo per S.Gia-
presso ai Pianetti; dai 300 
Aecydium crassum

- leurospermum.

Aethusa Bunius.

Allium pauiculatum.

Alnus incana.

Anthericum ramosum .

Arenaria fasciculala.

Arum maculatum, maculis nigris. Ai Pianelli.

Arundo sylvatica.

Athamanta Cervaria

- Oreoselinum.

Bolettis aereus

- c eciulis.

Bromus gigauteus

- secalinus.

Buphtalmum spinosum .

Bupleurum Gerardi.

Campanula patula

__ persicifolia

sibirica

- spicata .

Carduus defloratus.

Carex alba

- pedata.

Carliua vulgaris.

Carthanus lauatus.

Cenomyce alcicornis

- - furcata.

- sympliycarpa.

Cinicus olcraceus. Castione.

Coruus mascula.

Cynoglossum officinale.

Cyperus flavescens.

- fuscus.

- glomeratus W. Euum. non ejusd. Sp. pl. Presso Castione. ( C. culmo triquetro, umbella triradia-

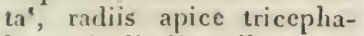
lis, spiculis linearibus confertissimis, cylindraceo-capitatis, involucris triphyllis umbella longioribus Willd. 1.c.Cyperus australis Schrad. Fl. germ. I. 116. Cyperus aquaticus italicus, procerior, locustis tenuissimis in race- mum dense rongestis, semiue cincreo longo perangusto. Mich. gen. 15. Segru. Ver.III. 65. (ab). 11. lig. 2.)

Cytisus capitatus

- hirsutus

- purpureus.

Diantlius carthusianorum - plumosus. $D_{e} C$.

Dictammus albus.

Enilocarpon miniatum .

Erythraca Centaurium .

Euphorbia nicaeensis.

Euphrasia lutea

—_oflicinalis

- tricuspidata .

Evonimus latifolius.

Festuca serotina.

Galium Bocconei.

Gnaphalium montanum.

Grimmia apocarpa.

Helleborus viridis.

Hieracium porrifolium

- staticifoliun .

Iuncus bufonius.

Lactuca perennis.

Linkia pulposa. Mihi. Collema pulposum. Achai.

Lecidea baldensis

- callosyne.

- lapicida. - Wulfenii .

Lepraria incana

- - B. L. latebrarum.

Limodorum abortivum . (Orobanche Calc. Viagg, 1x. )

Linum catharticunı

—— tenuifolium.

Malva Morenii . Milii . M. caule erecto, foliis subrotundo-cordatis, quinquelobis, crenatis, subus incanis, calycibus tomentosis . Pollin. Pl. nov. vel min. cogn p. 16.

Melissa officinalis.

Mentlıa arvensis.

Nepeta Cataria 
Nepeta nuda.

Ononis minutissima.

Orchis fusca. Segu. Ver. II. p. 122. n. 2. tab. 15. f. 2.)

- hircina. Nelle selvelic presso Castione. (Segu.Ver.II. p. 121. n. I. tab. I5. f. I.) Ornithogalum minimum. Fra le biadc.

\section{- umbellatum.}

Orobanche caryophyllacea

- elatior

- ramosa. Infesta i campi coltivati a tabacco.

Orobus niger.

Parmelia conspersa.

Phleum pratense. $\beta$. P. nodosum.

Pimpinella dioica.

Plantago subulata.

Poa rigida.

Prunella grandiflora

- vulgaris.

Pteris aquilina.

Rhamnus catharticus

- saratilis.

Rhus Cotynus.

Rosa arvensis.

Salix aurita

- triantra.

Satureja montana.

Scirpus palustris . Caslione, Brenionico.

- - - . minor, spica

Ai luoghi detti Canalette, Tolghe fino alla valle del Tretio lungo il torrente Aviana e il sentiero dei suppiadori, dai 1000 ai 1200 metri.

Arenaria bavarica. (Alsine alpina, foliis teretibus obtusis, flore albo. Sezru. Ver.I. 4ig. Pona Bald. ital.r 59.c.fig.) Parbula subulata.

Cnicus palustris . ovato-lanceolata, pauciflora. Ivi .

Sedum album

- reflexum.

Sempervivum arachnoideum.

Senecio erucifolius. Spreng. Fl. hal. p. 240. et Pers. Syn. pl. 435. Presso Mori. S. foliis pinnatifias dentatis supra glabriusculis, subtus tomentosis, perauthodiis apice coloratis, radio patente. Pollin. Pl. nov. vel min. cogn. P. 22. Jacobaca Senecionis folio incano, perennis.Segu. Ver.IIL.278. Aloren. hort.sicc. II forte non pertinet $S_{c n e-}$ cio erucifolius Limn. et W. Sp. pl.

Serratula tincloria.

Stipa pennata.

Thesium Linophyllum. Castione.

Triticum caninum .

Tussilago Farfara

- Petasites.

Urceolaria calcarca

- scruposa.

Uredo tussilaginis .

Veronica spicata.

Verrucaria Schraderi.

Erysimum Barbarea.

Hieracium staticifolium.

Potentilla caulescens.

Salix riparia.

Selinum Seguieri.

'Thesium alpinum.
Senecio viscosus.

Triglochin palustre. Presso

\section{Al prato di Brentonico, 1300 metri.}

Aconitum ccrnuum. Var. fl. al- bo-variegatis. 
Apargia hispicla

\section{- Taraxaci.}

A rusdo cpigejos.

Campanula Jarbata .

Centaurea phrygia.

Epilobium angustifoliun.

Gentiana lute:

utriculata.

Hypericum hirsutum

\section{- montanum.}

Sambucus racemosa.

Nella ralle dell Artillon,

\section{Aconitum certumum}

- var. Il. alho-variegatis.

-- Lycoctonum

Napellus.

Actea spicatia.

Agaricus alneus

- amaethysteus

campestris

- ceriuus

(coprinus) strictus

coriaccus

flammeolus. Mlihi. A.

pileo flansmenlo demmm concavo, lamellis firris, stipite croceo listuloso. Dullin. 'l'l. nov. vel $\mathrm{min}$. $\operatorname{cog} n$. [?. 3 .

- porrigens

- - steccorarius

vaccilius.

Anemone uemorosa

- - ranunculoides.

Asperula odorata

- - taurina.

Aspidium Filix focmiua

- Lonchitis. (Louchite a-

spera maggiore. Pona Bald. ital. 17r. 24\{, Lonclite aspera. Calceol. Viagg. 11.) Betonica alopecuros

- hirsuta.

Boletus lulescens

- - velutinus

- versicolor.

Bryum annotinum.

Cacalia alpina.
Selinum Sesuieri. (Ligusticum alpiuum perenne, ferulae folio, florihus albis. Sigull. Ver. II. p. /1. tab. 13.

Cl. Willdenowius in sui operis Specics plantrorum nuncnpati tom. I. p . Ifati. ctiam ad Ligusticume pyenaeum dubiam plantan falso refect phasem et lithulam Seguicri).

Scuecio laciniatus. Eeriolon.

Carclamine impatiens.

Carex baldensis: spica andro5y na, ifgregalo-rapilata, basi tribracteata, spiculis subscnis, lateralibus femineis, centralibus $x-3$ androgynis, flosculis inferioribus femiueis, terminalibus masculis, capsulis iuflatis. Milu. Içon. Ilost. Gramin austr. vol.IV. (a)!. 79 .

(Carex alpina, capitulis albis, squamosis, triquetris, et pulchre lucentibus. Segu. Ver.I. p. 125.

Gramen montanum junceum, capite squamoso. C. Buth. prods. p. 13. c. je.

,Tiadix repens, lignescens, fibris aucta, percunis. Culmi erecli, trigoni, glabri, laeves, tautum inferne foliorum vaginis vestiti. Folia canalicuJata, glabra, in brevem et tiquetrum cuspidem attenuala, ad oras, superiutemque carinae partem scabra. Spica romposita e spiculis tribus eirciter; latcralibus femineis; spicula terminali androgyna, inferne fininea; apice mascula. Siquamae superiores albae, ovatuc, rel oblongae, aphyllae, inferiores tres circiter, in folium 
desinentes: folio, squamam intirmam tcrminante longissimo. Fl mas. Filamenta tria, brevia. Antherac parvac obtusae. Fl. four. Germen ovatum, turgidum. Stylus brevis. Stigmata tria, villosa. Capsúla ovata, inflata. Semen triquelrum, Most. l. c. pag. $4^{5}$.

Questa bellissima Carice non cresce nè sulle altissime cime né sulle umili. Ama le mezzane e la plaga orientale e meridionale. E però rinviensi in tutto il fianco orientale, incominciando da valle Basiana, valle-fredda, poi alla Lonza, nelle parti inferiori di valle Losanna, clel Sassetto, alle Acque-negie, fino alle coste dell'Artillon).

Circaea alpina

- lutetiana.

Convallaria bifolia

- majalis

- verticillata.

Daphne Mezereum.

Dentaria bulbifera

- enneaphylla

- pentaphylla.

Digitalis ambigua

- lutea.

Doronicum austriacum .

Eryophorum polystachyum . Cinn. Willd. Sp. pl. Eriophorum latifolium. Schrad.

Galanthus nivalis .

Gentiana utriculata.

Graphys scripta.

Iyduum repandum var. alba .
Hypoum serpens

I- splendens.

Impaticns Yoli tangere.

Jungermannia complanata -_ Erharthiana. $W e b$.

Juniperus nana .

Lapsana foctida.

Jecanora albella

- varia $\varepsilon$. apochroea .

Leskea attenuata. Hedw.

Lonicera alpigena.

Lycogala miniata.

Lycoperdon pyriforme.

Oxalis Acetosella.

Paris quadrifolia.

Phallus impudicus . ( Fallo olandico. Pona Bald. ital. p. 225. )

Polyporus amorphus. Fries.

Pyrola rotunda

- - secunda.

Prenanthes muralis.

Pulmonaria augustifolia

- offiçinalis.

Ramalina fastigiata

- pollinaria.

Rosa alpina.

Rubus idaeus

_- saxatilis.

Sanbucus racemosa.

Sanicula europaea.

Saxifraga aizoides.

Senecio saracenicus

- laciniatus. Bert.

Sphaeria fuliginosa.

Thelephora cruenta.

'Tremella juniperina. In Junipero nana.

T'urritis glabra

— hirsuta.

Veratrum nigrum .

Ai luoghi detti le Acque-negre, e Campion, eguali altezze dell' Artillone . Arabis alpina . Arctium Lappa. Adianthum Capillus Veneris. Cnicus eryophorus

Atropa Belladonna. Boletus communis. 
Cnicus palustris.

Cyperus flavescens.

Epilobium hirsutum

- montanım .

Eryophorum polystachium.

Geum rivale.

Hypum commutatum.

- riparioides.

Juncus effusus.

Lycopodium clavatum

complanatum

Sclago.

Orchis bifolia. (Segu. Ver. II.

p. 125. n. 1'. tab. 15. f. 10) Pascula. (Segu. Ver. II.
P.12午. 125 . n.6.7. t. 15. f.5.6.)

Pinguicula flavescens. Flork.

- vulgaris.

Rhododendron ferrugincum

- hirsutum.

Salix aurita

- corruscans

- Pontederana

- riparia

- Wulfeniana.

Schoenus nignicans.

Scirpus Caricis.

Scrophularia nudosa

- verua.

Tussilago Farfara.

Lungo la via che guida ai Lavaci, e nelle foreste laterali sino al pian della cenere, dai 1000 ai

\section{400 metri.}

Agaricus flammeolus. Mihi.

Arenaria trinervia.

Aspidium aculcatum

3. minus

- Filis focmina

\section{Filix mas}

- fragile

Halleri.

Adianthum album Joannis

Bauhini, latifolia. Ponted.

Comp. 20. Segu. Ver.I. 7o.)

- Lonchitis.

Asplenium Adiauthum nigrum

__ Trichomanes

viride .

Botrychium Lunaria (Lunaria racemosa orvero minore del Mattioli. Pona i95. 2 ío. Lunaria del graspo. Calc. Viaggio 12. )

Cenomyce furcata

- - - $\delta$. recurva .

Ceterach officinarum .

Collema furvum.

Epilobium angustifolium

\section{montanum}

Epipactis Nidus Aris. Selva dei Lavaci ( Satirio abortiro del Lobelio. Pona Bald. ital. p. 238. )

Erysimum Barbárea .

Euphorbia dulcis

- sylvatica.

Gentiana lutea.

Hicracium praemorsum

-- staticifolium.

Hypnum recognitum. Ai $L a-$ v'aci.

Ligusticum peloponense. ( $\mathrm{Ci}$ cutaria fetidissima del Lobelin da altri chiamata Seseli Peloponense. Pona Bald.ital. 24o. Seseli peloponense. Calc. Viagg. 10.)

Mnium ligulatum. Brid.

- punctatum

Ophrys Moworchis . (Segu. Ver. II. $13 \mathrm{x}$. n. 22. t. 16 . fig. I5.)

Orchis albida. (Pseudorchis. Segic. Ver. III. p.25\%. Mich. gen. 3o. tab. 26. )

Pelidea aphtosa.

Polypodium Phegopteris. Pian della conere.

P. Iytrichum juniperinum 
Polytrichum vulgare.

Pyrola rotundifolia

- - secunda .

Ribes petraeum.
Rỉes Ura crispa.

Scandix odorata.

Triticum caninum .

Uvularia amplexifolia.

Nella selva d'Avio e lungo la via onde si scende al villaggio, dai 150 ai 900 metri.

Accr monspessulanum . Nelle Jungermania pubescens

selve sopra il villaggio.

- Platanoides

- Pseudo-Platanus .

Agrostis alba

- Calamagrostis.

Betula alba.

Cacalia alpina.

Crataegus Oxyacantha. W. Sp.

pl. Mespilus Ejusd. Enum.

Cytisus hirsutus

- nigricans

-

Daedalea quercina .

Daphne Laureola

- Mezereum.

Eyuisetum hiemale.

Festuca elatior W. Enum. F. arundinacea Schreb. Lungo

il torrente nella discesa .

Ilex Aquifolium.

Jungermania aspleniodes

- complanata

- dilatata

- platyphylla

Ai pascoli del Cerbiol e di monte Gambon, dai 1300 ai 1400 metri.

Aristolochia rotunda.

Arum maculatum. var. fol. immaculatis .

Chaerophyllum hirsutum

- - - . fl. purpureo.

Cytisus purpureus.

Ornithogalum luteum .

Ai pascoli e nei dirupi di Artaea spicata.

Adoxa Moscatellina .

Anemone ranunculoides

- trifolia.

Aquilegia vulgaris.
_- tamariscifolia.

Lunaria rediviva. Presso la Cateratta nella discesa. (Lunaria major, siliqua longiore. Segu. Ver. I. 379. Lunaria odorata maggiore degli erharj. Pona Bald. ital. 216. Lunaria odorata. Calc. Viagg. 9. Lunaria greca . Calc. Viaggio 13. )

Milium effusum.

Ostrya vulgaris.

Pohlia pallens. Brid.

Populus tremula .

Prunus Avium.

Pyrus Amelianchier

- Malus. Nelle selve sopra il villaggio.

- Pyraster. Ivi.

Sambucus nigra.

Tilia europaea

-T microphylla.

Ulmus campestris

- - var. latifolia montana.

Peltidea canina

- horizontalis.

Plantago montana.

Scrophularia canina

Stachys alpina.

Noveza, 1500 metri. Arabis alpina.

Arenaria tenuifolia.

Asperula odorata

- - taurina .

Betonica hirsuta
- verna. 
Betonica oflicinalis.

Bovista plumbea.

\section{Carduus eriophorus}

montosus . Milhi.

Con tal nome ì descritto nel primo fascicolo delle piante nuove o poco conosciutc una specie di Cardo frequente in tutti i pascoli alquanto elevati di monte Baldo e dei monti Veronesi, Bresciani, 'irolesi, Vicentini, e Comaschi. Di un cotal Cardo avendo spedito esemplari a varj mici amici corrispondenti, e domandati del parer loro, quale mi rispose essere il Curduus alpestris Waldst, e Kitaib., quale il C'ardius ciefloratus, e quale il Carduus crassifolius Hornem. Differisce però dal Cardo alpestre per le foglie non mezzoscorrenti, verdi in ambe le pagine, pennatofesse, a laciuie bilobe cigliato-spinose, ma affatto scorrenti, glauche ossia verdiccie per di sotto, colle lacinie o semmenti inegualmeute tridentato-spinosi. $\dot{E}$ diverso dal Cardo disfiorato perchè nou à le foglie radicali indivise, e le cauline non mezzoscorrenti pennatofesso-seghettate; dal Cardo crassifoglio differisce poi per le foglie non rezzoscorrenti. Pero dai semi del Cardo erassifoglio ricevuto dal chiarissimo Sprengel ò ottenuto piante a foglie affatto scorrenti al pari del mio Cardo montanesco, sicchè poteasi conchiudere appartencre alla stessa specie. Pure coltivati per due anni nell' Orto botanico Veronese infra le piante alpine e il mio Cardo montanesco e il Cardo crassifoglio nato dai seıni dello Sprengel ne ì notata la seguente differenza. 'Iutta la pianta del mio Cardo è liscia verde ad eccezione delle foglie che sono inferiormente glauche: sono esse sinuose a semmenti tridentato-spinulosi con denti ineguali. Le corolline sono d'un porporino gajo. Nel Cardo erassifuglio l' intera pianta è glauca, e le foglie sono per di sotto d' un verdiccio più pallido, e oltracciò a lacinic bidentatospinulose: le corolline sono d'un porporino smorto; onde presoita un aspetto diverso. Contuttociò non $\mathrm{mi}$ pajono caratteri suflicienti a determinarli come specie distinte. In ogni modo cccone le frasi :

Carduus crassifolius : foliis decurreutibus glabris, glaucescentibus, oblongo-lanceolatis, sinuatis, laciniis inacqualiter bidentato-spinulosis, pedunculis pubescentibus monaulhodiis, Jracteis peranthodialibus patentibus mucronatis. Mihi .

Cardus montosus: foliis decurrentihus glabris, subtus glaucis, oblongo-lanceolatis, sinuatis, laciniis inaequaliter tridentato-spinulosis, pedunculis pubescentibus mouanthodiis, bracteis peranthodialibus patentibus mucronatis. Mihi.

Ma poiché si favella di Cardi non debbo tacere una cosa che risguarda l'altoro Cardo nella stessa mia operetta de- 
scritto col nome di Carduus sumanus. Fra i Botanici che videro tale specie altri disse poter essere il Carduis crassifolius Hornem., altri il Cardures pannonicus Linn. Ma il mio Cardo, da quanto arlducemmo dianzi, scorgesi non poter essere il C. crassifoglio. Non il C. pannonico pel caule non lanuginoso ma liscio; per le foglie non inermi ma inegualmentè spinuloso-cigliate e glauche inferiormente; per gli antodj più voluminosi di quelli della Serratula arvensis, per le brattee perantodiali non segnate da una linea bianca, nè inermi, ma verdi, mucronate e aperte; e infine per le corolline d'un porporino vivace e non pallido. Parrebbe piuttosto il Cardures transalpinus del Suter. 'Tuttavolta comechè da me non veduto, da quanto traesi dalla descrizione pare differire il mio Cardo sumano da esso per le foglie nou macchiate e scorrenti, ma tutte mezzo-scorrenti, e glauche solo per di sotto, pei peduncoli non incrassati, e per le brattee perantodiali non erette ma affatto aperte.

Chrysospleninm alternifolium. Cerastium coespitosum. Kitaib. Circaea alpina

\section{- luteitana.}

Corydalis bulbosa.

Crocus vernus. (Croco verno primo del Clusio.'Pona Bald. ital. pag. 239. Croco marzio. Calc. Viagg. Io.)

Dentaria enveaphyllos

- pentaphyllos.

Doronicum austriacum .
Epipactis ovata.

Erigeron alpinum .

Euphorbia ambigua. (Folia, involucra, et involucella integerrima, umbellae radii crecti, capsulae nudae verrucosae; quibus caracteribus differt ab affinibus $\boldsymbol{E}$. diclci et E. carniolica. Mihi occurrit etiam in monte Sumano.

Festuca documbens. (Danthonia. DeC. Gaud.)

Galanthus nivalis.

Galium austriacum .

Galeobdolon luteum.

Hepatica triloba.

Iris graminea.

Ligusticum peloponense.

Lycoperdon giganteum

_- pyriforme $\beta$. tessclatum.

Mercurialis annua

-

Muscari botryoides.

Orchis bifolia (Segu. Vcr. II. p. 128 . n. 14. tab. 15. f. 10.)

Orobus luteus.

Oxalis Acetose!la.

Phleurri alpinum.

Polygala amara

- Chamaebruxus 。

Potentilla argented

- aurea.

Ranunculus aconitifolius

_- lanuginosus

montanus .

Saxifraga rotundifolia.

Selinum Carvifolia.

Senecio laciniatus. Bert.

Solidago Virgaurea

$$
\begin{aligned}
& \text { B. Solidago alpe- } \\
& \text { stris. W. }
\end{aligned}
$$

Spergula saginoides.

Stellaria graminea

- nemorum.

Teucrium Botrys.

Tormentilla erecta. 
Valeriana montana

\section{- tripteris.}

Veronica urticaefulia.

Viola biflora
Viola canina

—_ montana

- tricolor $\beta$.
Achillca aubigua. Mili. A. folis pimnatis pubescentibus, pinuis piunatilidis inciso dentatis, corymbo composito. Pullin. Pl. nov. vel mia. $\operatorname{cog} n . p \cdot 2 \%$.

Nou ui soccorre il luogo preciso ove ò rinrenuto quesia bclla specie, ma parmi sia lit valle Losanna.

Achillea ligustica.

Allium ochroleucum .

Arabis saxatilis.

Aruica scorpioides.

Aspidium fragile

- - B. Aspidium Pontederae. W.

\section{- rigidum.}

Boletus fuligineus.

Bupleurum graminifolium .

Campanula rotundifolia

__ _- B. Campanula pusilla. Jacr.
Carex baldensis.

Chrysanthemum Leucanthemum $\beta$. montanum .

Corydalis lutea .

Globularia nudicaulis.

Polygala amara.

Pyretrun clegaus. Mitie. P. foliis pinnatis multilidis, laciniis linearibus acutis supra convexis subfus canaliculatis, pappo quadricienta. to. Pollin. Pl. nov. vel mia. cogुn. p. 24.

Saxifrma sedoides.

Toficldia palustris.

Urer's Aspiciii. Mitii. U. glohosa sparsa vel oblonga confluvens flavescens. p., ilin. Pl. nov. vei mia. rugn. p. 33. In Aspidil fragilis var. 5 . An varietas $U_{i}$ iclinis Polypodii. Pers. Syn. Lugg. p. $217, ?$

Nei prati e campi diclla Ferrara crescono Je seguenti, la piu parte celle quali si rinvengono anche al Prabazar e negli aitri pascoli di mezzana altezáa; dagli 800 ai Icoo metri.

Aclillea distans

j. 11. purpurascente

Millefolium

Agrostis alba

- vulgaris .

Aira caryopliyllea

- cespitosa

- cristata flexuosa .

Ajuga reptans. Alchemilla vulgaris . Anthoxanthum odoratum .
Anthyllis vulneraria.

Apargia alummalis

- crispa

- hastilis

__ hispida.

Arctium I appa.

Arabis alpina.

Asphodelus albus.

Astrantia major.

Avena flavescens

- pratensis.

Perberis vulgaris.

Letouica officinalis 
Betonica officinalis $\beta$. A. albo . Biscutella apula.

Briza media.

Bromus erectus

_- mollis

- secalinus.

Campanula glomerata

_- Ranunculoides

- rotunda

- Trachelium.

Carex collina

- distans

- hirta

- pedata

- recurva.

Carum Carvi .

Centaurea Jacea. B. C. amara

- montana

- - nigrescens.

Cerastium aquaticum

_- ciliatum. Kitaib.

Chacrophyllum hirsutum

- temulum

Chelidonium majus

Chrysanthemum Leucanthemum .

Clematis recta

- Vitalba.

Cnicus Erysithales.

Colchicum autumnale.

Conium maculatum.

Cucubalus Behen.

Cynosurus cristatus .

Cytisus capitatus.

Dactylis glomerata.

Daucus Carota.

Dianthus atro-rubens.

Equisetum arvense

-._- fluviatile.

Euphorbia dulcis

\section{- sylvatica}

- verrucosa.

Euphrasia officinalis.

Festuca clatior. W. Enum.

- duriuscula

—- oviná

- prateusis.

Galeopsis cannabina
Galcopsis grandiflora

-_ Ladanum

- 'Tetrahit . Variat eanle glabro, et hirto, corolla lutea, vel alba, vel purpurca, vel purpureo-variegata.

Galiun Bocconei

- purpureum.

Genista ovata

- pratensis. Mihi. G. ramis adscendentibus striatis, foliisque oblongo-lanceolatis hirsutis, corollis et leguminibus glabris. Pollin. Pl. nov. vel min. cogn. p. 19.

Gentiana acaulis

- cruciata

- germanica

- verna.

Geranium phaeum

- pratense

_- robertianum

_- rotundifolium

- sanguineum.

Geum rivale.

Gnafalium dioicum $\propto$. fl. purp. - - - $\beta$. fl. albo.

Hemerocallis Liliastrum.

Heracleum flavescens. W. H. angustifolium . Jacq. Fl. radiati albi. (Sphondylum majus aliud, laciniatis foliis . Segu. Ver. III. 226. Moren. hort. sicc. Sfondilio con foglia laciniata. Pona Bald. ital. Igo. 2ri.)

Heracleum Sphondylium

- - $\beta$. fl. purpureis.

Holcus avenaceus. Schrad. Avena elatior Linn.

_- lanatus

- odoratus.

Hypericum hirsutum

- montanum

- perforatum.

Hypochoeris radicata.

Inula hirta

- - salicina 
Inula syuatrosa.

Juncus acutillorus

- bufonits

- eflusus.

Lamium album

- maculatum

\section{Orvala .}

Lathyrus pratensis.

I,contodon Taraxacum .

Leonurus Cardiaca .

Lilium bulbiferum

Martagon .

Linum catharticum

- - tenuifolium

- viscosum.

Lolium perenne

Luzula campestris

- maxima

_- pilosa

- spicata.

Lychnis Flos Cuculi

- sylvestris.

Medicago falcata

- L I upulina

- sativa.

Melampyrum arvense

- cristatum

nemorosum

- pratense.

Melica coerulea . Melissa officinalis

- pyrenaica.

Melittis Melissophyllum.

Mentha arvensis

- sylvestris.

Muscari botryoides.

Myosotis scorpioides.

Orchis conopsea

- globosa

- mascula. (Segu. Ver. II.

p. 124. 125. n. 6. 7. tab. 15.

f. 5. 6. )

- Morio

- piramidalis. ( $S$ egu. Ver.

II. p. 129. n. 15. 16. tab. 15 .

fig. II. )

Ornithogalum narbonense

pyrenaicum
Ornithngalum umbellatum .

Orobauchic caryophyllacea.

Orobus luteus

- niger.

Paconia corallina

- oflicinalis.

Panicum glaucum

- verticillatum.

Pastinaca sativa .

Pedicularis comosa.

Phleum alpinum.

Phyteuma orbicularis.

Pimpiuella magna

- Saxifragat.

Plantago lanceolata.

- media

- montana.

Poa alpina

- - 3 . vivipara

- pratensis

- trivialis.

PoIygonum Bistorta

___ viviparum.

Potentilla alba

- - argentea

- aurea .

Primula veris.

Prunella grandiflora.

- vulgaris .

Prunus Avium .

Pyretrum corymbosum.

Ranunculus bulbosus

- lanuginosns.

Rhinanthus Crista galli.

Ribes Uva crispa.

Rumex Acetosa

- alpinus

-.. obtusifolius.

Salvia glutinosa

—_ pratensis.

Scabiosa Succisa

- sylvatica.

Scilla bifolia.

Sedum acre

- atratum

— dasyphyllum

_- reflexum

- rubens. Milic. Crassula 
rubens W. Stamina semper decem.

Sedum sexangulare.

Sempervivum tectorum.

Sesleria coerulea.

Silene nutans.

Solorina saccata.

Spiraea Aruncus

- Filipendula.

Stachys recta

- sylvatica.

Stellaria crassifolia

- graminea

- Holostea.

Taxus baccata. In valle $O_{i} \approx a$.

'Teucrium Chamaedrys

- Botrys

- montanum .

Thalictrum aquilegifolium

- minus.

Thesium Linophyllum .

'Thlaspi arvense

- campestre.

Thymus alpinus.

Nei luoghi detti la Lonza, Campedello, Valle brut$t a$; dai 1200 ai 1300 metri.
A $^{\text {llium paniculatum }}$

Ar butus Uva Ursi. In copia sulle alte pendici.

Euphrasia tricuspidata ( E. angustis et tricuspidatis foliis, floribus ex albo purpureis.

Zannon. hist. I 10. tab. 76.) Globularia nudicaulis .

Melica coernlea'.

Nella valle-fredda, e specialmenite nelle rupi che le sovrastano fino alla valle di Navole, dai $x 400$ ai I65o metri .

Achillea nobilis .

Aconitum Anthora

- cernunm

- Lycoctonum

-napelius.

Ophrys Monorchis.

Rubus idaeus.

Rumex scutatus. (Osalide con foglia rolonda. Pona Bald. ital. 247 . )

Salix ambigua

_- corruscans

- Wulfeniana.

Sedum acre

- atratum.
A - $\beta$. bulbiferum .

$\mathrm{Ca}^{\text {rex baldensis . }}$
Tilia europaea

- microphylla .

Tragopogon prateuse.

Trichodium caninum

Trifolium alpestre

- montanum

- pratense

- repens

'Trollius europaeus.

Turritis glabra

- hirsuta.

Ulmus campestris .

Valeriana officinalis

V- tripteris.

Verbascum Lychnitis

- ni grum.

Veronica officinalis

- - serpillifolia.

Viola canina

- tricolor $\alpha$.
- rubens.

$-\ldots$. 


\section{Anica Dellidiastrun}

- montana.

Athamanta cretensis .

Botrychium Lunaria.

Cares baldensis

capillaris .

Cyperoides alpinum, spicis seminiferis pendulis, binis in summo caule. Segu. Ver. III. p.S3. tab. 3. f. $\mathrm{x}$.)

\section{Centaurea montana}

- Rhapuntica . (Centaurea seconda maggiore del Clusio, da al tri creduta la maggiore di Dioscoride, cla altri chiamata Reu baldense. Pona Bald. ital. I; I.)

Cerastium latifolium.

Chara hispida. Nella fossa ore si abbeverano gli armenti.

Clirysantliemum Leucanthemum $\beta$. montanum .

Cnicus acaulis.

Cucubalus Behen $\beta$. montanus.

Daphne Cineorum.

Tianthus plumosus $D_{e} C$.

Dicranum scoparium.

Digitalis ambigua.

Draba aizoides.

Epilobium angustifolium

- montanum $\delta$. E. alpestrc.

Euphorbia ambigua.

Gentiana lutea (Genziana Pona Bald. ital. $1 \mathrm{~S}_{9}, 2$ I. Calceol. Viagg. 12. 14.)

Globularia nudicaulis.

Helianthemum grandiflorum

- oclandicum.

Hicracium cymosum

villosum.

Juniperus nana.

Laserpitium latifolium

- peucedanoides.

Linum alpinum.

Narcissus poeticus.

Ophyoglossum vulgatus .

Ophrys alpina.

Orchis viridis
Orchis sambucina a. ft: luteo . ( Segul Ver. III. p. 2/9. u. 7. tab. 8. lig. 5.)

Orobus luteus.

Pacderota Bonarotta.

Pedicularis comosa

- tuberosa.

Peplis Purtula. Nella fossa.

Phyteuma comosa

- spicata.

Pimpiuella dissecta .

Pinguicula flavescens. Flök. Calcar fere rectum, corolla alba, palato flavo. (l'inguicula di fior bianco. Pona Bald. ital. 2任.)

- vulgaris. (I'inguicula di for cerulco. Pone Bald. ital. 187.2 . 3 . )

Pinus Punilio.

Potentilla caulescens.

Primula Auricula

- carniolica.

Pyretrum corymbosum.

Rihamuus pumilus.

Khododendron hirsutum .

Rubus saxatilis.

Ruscus Hypoglossum. (I Ipoglosso. Ponc Bald.ital. 172.)

Salix ambigua

- corruscans

- Wulfeniana.

Saponaria ocymoides. Sasifraga Aizoon e. Stern\}.

$-\ldots$ coesia

- Ponae $\boldsymbol{u}$.

Scorzonera rosea. (Scorzonera angustifolia subcoerulea.Segre. Ver. III. 275. Scorzonera quinta ongarica del Clusio. Pona Bald. ital. 174. 211.) Senecio Doronicum

__ - var. $\beta$. (Jacobaea integro et crasso Hieracii folio. Segu. Ver. III. 2;8. ) Silenc Saxifraga. 
Soldanell montana.

Spartium radiatum.

Stachys alpina.

Thalictrum minus.

Thesium alpinum.

Tofieldia palustris.

Valeriana montana

- saxatilis.
Veratrum alloum

- nigrum .

Veronica aphylla

- fruticulosa

— saxatilis

- - serpillifolia .

Viola biflora

- tricolor $\beta$.

Alla fontana di Navole e nella prossima valletta detta del Bastion oltre molte delle diauzi nominate; 1700 metri incirca.

Aster alpinus.

Conjugata porticata. Vauch. Ectosperma baldensis. Mihi. (Conferva baldensis filamentis viridibus simplicibus et ramosis inarticulatis cylindricis obtusis, globulis gemmiferis solitariis, rotundis,

sessilibus breviterve pedunculatis, lateralibus et terminalibus. Pollin. PI. nov. vel min. cogn. p. 32.)

Mnium fontauum.

Orchis nigra.

Parnassia palustris.

Nelle valli Basiana ed 1 me, dai r200 ai r30o metri.

Agaricus campestris.

Arum maculatum var, immaculata .

Aristolochia rotunda. (Aristolochia rotunda vera. Pona bald. ital. 13.. Calc. Viags.9.) Barbula cirrhata

_- inclinata. Schw ägr.

Bryum annotinum.

Carex alpestris

- - baldensis

- clandestina

- ferruginea

- strigosa.

Comarum fragarioides .

Conferva aurea. Dill

Cynontodium Hedw.

Cytisus Laburnum.

Epipactis latifolia

- rubra.

Gentiana cruciata

- - $-\beta$.

Hypnum megapolitanum.

Jungermannia furcata
Jungermania pubescens .

Laserpitium peucedanoides.

Nepeta nuda.

Orchis odoratissima .

Polygala amara.

Polytrichum commune

- - juniperinum

—_ undulatum.

Rubus idaeus.

Saxifraga exilis. Mihi. S. caule subunifloro, foliis alternis, lineari-laneeolatis, radicalibus in rosulau dispositis, spathulatis. Pollin. Pl. nov. vel min. cogn. pag. II.

Scabiosa Succisa.

Scirpus acicularis. Nel margine della fossa ove si abbeverano gli armenti.

Sedum atratum.

Spallanzania Agrimonoides: foliis radicalibns pinnatis, caulinis ternatis, impari majore basi cunciformi . Pollin. Pl. nov. vel min. $\operatorname{cog} n$. p. 1 o. f. $r$ 
Sparganium ramosum. Nella fossa.

Symphitum tuberosum.

Nelle praterie del Prabazar e de' Crosati (dai rooo ai I 00 metri), oltre la più parte di quelle dei contorni della Ferrara, crescono

Aruica montana.

Una specie di Astragalo rinrenne il Martini nel Praba$z a r^{\circ}$, come traesi dal suo Catalogus planturum inventurum in ilinere montis Baldi. ( Veronae ex Typographia Jo. Berni 1 zo . ) Non riescendomi riferire tale specie a nissuna delle a me note, dal nome del primo, che ne dic la figura, io l'ó chiamata

Astragalus Clusii.

(A. caulescens erectus, foliolis lineari-lanceolatis pubescentibus, spicis elongatis confertis pedunculatis terminalibus, leguminibus nudis canaliculato-triquetris mucronatis. Mihi.

Onobrychis spicata folio pinnato, flore papilionaceo tetrapetalo pallido. Martini . Cat. pl. M. Baldi . Segu. Ver. II. 351 .

Onobrychis quibusdam flore pallido vel Polygalon. I. Bauh. Hist. II. 337. c. ic.

Onobrychis spicata floribus pallidis, nigris radiis notatis. C. Bauh. Pin 350.

Onobrychis tertia. Clus. Hist. CCXXXIX. c. ic.
Radix perennis lignosa ramosa, albicans. Caules plures sesquicubitales erecti. Folia impari-pinnata, $12-15$ - juga, fonuginosis. Pedunculi foliis ctea lineari unicuique flori longam confertam tcrminalem subsessiles, erectiusculi; petala albo-luteola striis nigricantibus notata, vexillo alis duplo longiore. Legumina nuda bilocularia. Semina Trigonellae Foenigraeci sed minora et nigra).

Carex collina

- flava

- pallescens

- Scopoliana

- tomentosa.

Carlina acaulis.

Convallaria multiflora.

Festuca flavescens. Bellard. F. varia Host.

- spadicea .

Gentiana ciliata

- utriculosa.

Hemerocallis Liliastrum.

Malva Morenii.

Selinum Chabraci.

Verrucaria pyrenophora. liolis lineari-lanceolatis, laduplo longiores striati, brasupposita. Flores in spican

\section{Dai Coltri discendendo al Santuario della Corona} lungo le scale e nelle selve e rupi adjacenti sino al villaggio e alla valle di Brentino (dai 150 ai 900 metri) le principali piante ch'ivi crescoro sono le seguenti : 
Acer platanoides

_- Pscudo-Platanus .

Actaca spicata.

Aecydium rhamni.

Aethusa Bunius

Cynapinm .

Agrostis arundinacca.

Allium carinatum

-_ rotundum.

Althaea hirsuta. Nella valle di Brentino.

Alyssum rupestre. (Tlaspi alpino petreo con aspetto di Míagro. Pona Bald. ital 185. c. fig.)

Andropogon halepensis: "panicula florente patula, flore hermaphrodito sessili, glumellaeque palcola exteriore aristata, masculis pedicellatis muticis. Milii.

Questa gramigna detta dal Linneo Holcus halepensis, dai 'Toscani Saginella selvatica - Cannerecchia, e da' contadini Veronesi Nalgastro o Melgastro, infesta i campi migliori dei colli e del piano della provincia nostra e delle vicine, moltiplicandosi prodigiosamente colle sue $\mathrm{grosse}$ radici striscianti. Tali radici ànno un sapore dolcigno mucilaginoso nou ingrato, e in alcune spezierie di Verona corrono sotto il nome di Smilace dolce e di Gramignone. Fu da me e da qualche collega usata piì volte con vantaggio nelle malattie reumatiche e artritiche non meno che nella sifilide, ora sola ora combinata ad altri farmaci in sostituzione e alla dose stessa della radice di Cina, della Smilace aspera o Rovo Cervione, della Dulcamara e della Salsa- pariglia. Ben ì vero, che non ispiegò la possanza di quest'ullima, ma si mostrò in varj casi piì attiva delle prime, e oltraccio riesce meno discara al palato. Non reputo quindi cosa vana il proporre ai pratici lo sperimento di questo indigeno rimedio ).

Angelica sylvestris.

Anthericum Liliago. Alle falde del Monte, ed alla Chiusa . Anthericum ramosum.

Apargia crispa

_- hispida incana .

Arabis Turrita.

Arenaria bavarica .

Arnica Bellidiastrum.

Artemisia campliorata.

Aruudo epigejos.

Una graziosa gramignetta $f_{1}$ rinvenuta dal Sig. Gebhard, che percorse tredici anni fanno il monte Baldo in compagnia dell'Arciduca Giovanni d'Austria. Ne ignoro il preciso luogo. Fu descritta dallo Sprengrel nel primo pugnello delle piante meno conosciute, e da me che ne ne ottenni da quest' ultimo esemplari. Il nome impostole è

Arundo pygmaea: culmo herbaceo, panicula spicata, glu mis bifloris, glumella triaristata brevioribus, arista dorsali paleolis duplo longiore. Milii.

Ex radice fibrosa culmus uncialis quinquenodis, inferne vaginis foliorum canescentibus obtectus. Folią linearia complicata pubescentia. Pili loco ligulae. Panicula spicata. Glumae paleae valde 
incequales; palea externa lanceolato-acuminati, scariosa, trinervosa, nervis medium versus evanescentibus; interna lineari-lanceolata uniucrvosa. Glumellae palcolac gluma longiores subaequales, externa apice dorsogne aristata, interna apice; arista terminalis paleolis duplo brevior, recta; dorsalis duplo lougior, recurra. Pilorum barba ad hasin glumellae pedicellata, pilis ciliatis glumella brevioribus. Pollin. Pl. nor. vel min. cogu. p. 4 . Asplenium Ruta muraria

\section{_- Trichomanes.}

Aspidinm fragile

_ _ Filix foemina

Filix mas.

Athamanta cretensis.

Dartramia Oederi.

Biscuteila apula.

Bromus asper

- - erectus.

Puphtalmum granciflorum.

Bupleurum baldense. Negli spinetti lungo l'Adige presso Rivole.

( B. caule erecto ramoso, foliis lanccolato-liuearibus, iuvolucris subquadribracteatis inaequalibus umbella brevioribus, involucellis quinquebractcolatis. Pollin. Pl. nov. vel min. cogn. pag. 7. )

- - ranunculoides. Lungo le scale.

Byssus antiquitatis.

Campanula petraca: caule declinato; foliis ovato-lanceolatis obtusiusculis, undulatis, crenatis, sublus tomentosis, floribus sessilibus capitatis et glomeratis, corollae laciniis oblique revolutis. Pollin. Element. botan. tom. II. p. rír. tav. 5. fig. 1. (Campauula foliis lanceolato-ovatis, serratis, hispidis, floribus per caulem dense congestis. Segu. Ver. I. P. I79. Trachelio maggiore petreo. Puna Bald. ital. 16 r. c. fig. catt. )

Campannla rapunculoides.

__ rotundifolia

- sibirica

- spicata

- Trab $\beta$. Albo

Carex clandestiua Drymeja.

Carpinus Betulus.

Caucalis Anthriscus

_- nodosa. A Prentino.

Cenomyce coccifera $\gamma$. asotea.

Cheiranthus erysimoides.

Choerophyllum temulum.

Circaea lutetiana.

Convallaria bifolia

__ majalis

- Polygonatum

___ verticillata.

Cornus mascula

- sanguinea.

Coronilla minima

—_ valeutina.

Corydalis lutea.

Cyclamen europacum .

Cynoglossum officinalc.

Cynonthodium capillaceum.

Cytisus alpinus

- C hirsutus

- Laburnun

- nigricans

sessilifolius .

Diantlius plumosus. $D_{e} C$. e Spreng. Pl. min. cogn. pug. II. 6 . Dianthus alpestris. Sternb. Reise in die Rhet. alp. 5o. Dianthus monspessulanus. Li,ln. Amacn. acad. IV. 3×3. Caryophyllus silvestris alter, flore laciniato 
odoratissimo. Segu. Ver. I. Radix annua sublignescens, ra437. et Moren. hort. sicc. mosa, extus flava. Caulis unon C. Bauh. Pin. D. foliis linearibus nervosis flaccidis, floribus sparsis solitariis, bracteis calycinis lanceolato-linearibus patentibus tubo parum brevioribus, petalis subbarbatis multifido-incisis. Nelle selve lungo le scale, e in tutti i colli Veronesi, Vicentini, Tirolesi e Bresciani.

- - sylvestris.

Dicranum scoparium.

Dictamnus albus.

Digitalis lutea.

Dorycnium lierbaceum .

Echinops Sphoerocephalus.

Endocarpon miniatum.

Epipactis latifolia.

Erica herbacea .

Eryngium amacthystinum.

Erysimum hieracifolium. Nella valle di Brentino. E. foliis lanceolatis, subsinuato-dentatis, siliquis erectis. Mihi. Hesperis leucoii folio serrato, siliqua quadrangula. Segu. Ver. I. 3S2. Moren. hort. sicc. Leucojo giallo montano. Pona Bald. ital. 232. c. fig. cattiva.

(Caules ex una radice plures vel caulis unicus ramosus ercctus, angulosus, scabriusculus, 1-2 pedalis. Folia scabriuscula sacpe sinuato-dentata. Siliquae tetragonae sub1) ubescentes erectae, stygmate subemarginato coronalae. Biennis. An varietas C'heiranthi crysimoidis?

Euphorbia dulcis.

-_- maculata. ( E. dichotoma, foliis oppositis oblique cordato-oblongis serratis maculatis, floribus axillaribus solitariis, capsulis laevibus.Mihi. nicus, pedalis et ultra, te.. res, scaber, dichotomus, ramis patulis vel saepe prostratis, superne atro-rubentibus. Folia breviter petiolata, opposita, oblique cordato-oblonga, obtusa, serrata, altero latere maxima parte integerrima, tri-raroquinquenervia, pilosiuscula, paginae superioris disco macula atro-purpurea notato, quandoque tota pagina purpurascente. Flores peduncuJati solitarii axillares. Involucrum (Calyx Linn.) octodentatum, dentious alterwis exterioribus (Petala Linn.) subrotundis, externe purpurascens. Ovarium glabrum, non verrucosum.

Questa Euforbia creduta indigena deli'America settentrionale cresce volgarissima ne" campi dei colli e dell'alta pianura Veronese come nella valle di Brentino, ncila valle Pulicella presso l'OspediaJelto e S. Ambrogio, nei colli d'Avesa e del Tagliaferro, alla Ca dei Cozzi, nella valle d'Illasi, ec. Fiorisce dalla melà di Agosto alla metà di Settembre, e in Ottobre matura il finto).

Euphrasia lutea

- officinalis

Favolus.

CARATTERE GENERICO

Pileus (dimidiatus) suberosns subtus in cellulas hexagonas exfossus. Mitii.

Favolus Mori. Mihi. Hexagonia Hori. Pollin. Pl. nor, vel 
min. cogn. p. 3j. lig. 2.3. CIRATTERE SPECIFICO.

F. pileo planiusculo, coriaceosuberoso, luteo et glabro. Mihi. Sui rami del Moro bianco moriente presso Bicnlino.

Ferula nodiflora.

Festuca elatior W. Enum. F. aruudinacea. Scheb. duriuscula

- glauca. Lam. Schiad. ovina

- 3 . spiculis muticis. F. tenuifolia. Hoffm.W.Enum. pinuata pratensis. Huds. WV. Enum. F. elatior. Linn. Fissidens adianthoides Fragaria collina.

Funaria liygrometrica.

Galium sylvaticum.

Genista germanica .

Gentiana asclepiadea

- cruciata.

Geranium robertianum

--- sauguineum .

Geum urbanum.

Clobularia cordifolia

- nudicaulis

- vulgaris.

Gratiola officinalis . Lungo l'Adige.

Gymnostomum tenue.

Gypsophyla Saxifraga .

Helianthemum Fumana

- marifolium

- - vulgare.

II racleum Sphondylium . llieracium amplexicaule

- - incanum

- murorum

- praemorsum

- porrifolium var. fol. glal)ris integerrimis. Holcus odoratus. Hypericun Androsacmum - hirsutum.
Hypocrepis comosa.

Hypum abietinum. Brid.

- arbuscula. Brid. H. alopecurum Hedw.

_- crista castrensis. Medir.

- cupressiforme

- splenders. Hedir.

- triquetrum.

Inula hirla

- sefuarrosa.

Iris sambuciua. A lato a un antico castcllo lungo la strada del porto della Pilarole.

Jungermania asplenoides

- complanata

- dilatata

- furcata

-- pinguis. Nelle pareti del-

la fonte dei Coltiv.

- platyphylla

- jubescens.

Juniperus communis .

Lacluca perennis.

Lamium Orvala.

Laserpitium Siler.

Lecanora chloroleuca

- circinnata

- galactina

_- milvina

- murorum

-.. parella

- versicolor

- Villarsii.

Lecidea haldensis

- callosyne

- confiuens

- - B. pillularis

- Lapicida i). cyauea

_- marmorea $\beta$. L. cupularis

- plocina

- Wulfenii.

Leersia lanccolata.

Lepraria incana $\alpha$.

Leskea complanata. Hedw.

- sericea. Hedir.

Limodorum alıortivum.

Ijuhia pulposa Mini. Collema pulposum. Shlaw. 
Lonicera Xylosteum.

Luzula nivea.

Lycopodium helveticum.

Melampyrum cristatum

- nemorosum.

Mclica ciliata

- nutans

—_ uniflura.

Melissa officinalis.

Melittis Melissophyllum.

Mercurialis peremnis.

Mespylus Cotoneaster

- - germauica.

Innium cespititium . Brid.

- cuspidatum . Brid.

- serratum. Brid.

Mölringia muscosa. (Alsine tenuifolia muscosa. Segu. Ver. I. 418. tab. 5. fig. I. Ubi flos male exprimitur pentapetalus).

Neckera crispa. Hedir.

- curtipendula. Hedir.

- viticulosa. Hedir.

Ononis minutissima

- - pinguis.

Onosma echioides.

Opezrapha calcarea.

Orchis conopsea

- maculata. (Segu. Ver. II. p. 132. n. 24 . tab. 15. f. 16.)

Orobanche caryophyllacea.

Orobus niger

- vernus.

Ostrya vulgaris.

Pacdcrota Bonarotta.

Paconia corallina.

Parmelia caperata

_- olivacea

_- saxatilis .

Pedicularis comosa.

Peltidea horizontalis.

Philadelphus coronarius.

Phytcuma comosa

- orbicularis

- spicata.

Picris hicracioides.

Pimpinella dioica
- dissecta. Fe' dirupi a sinistra del Santuario. ('I'ragoselinum minus. Segiu. Ver. III. 2 I9. Moren. et Bordon. hort. sicc.)

_- _- - umbella purpurascente.

Saxifraga .

Pistacia Therebinthus.

Plantago lanceolata (s.

— subulata.

Poa compressa

- laxa

_- nemoralis

- pilosa

rigida.

Polysperma glomerata. Vauch. Nel fonte.

Polytrichum commune juniperinum .

Potentilla caulescens.

Prenanthes muralis

- purpurea.

Prolifera parasitica . Vauch. Sulla Polysperma glomerata.

Prunella grandiflora

- vulga ris

_- _ $\beta$. fl. albis

- - $\gamma$. Prunella intermedia .

Prunus Avium

- Mahaleb.

Punica Granatum .

Pyretrum corymbosum.

Pyrus Amelanchier

- Aria

- torminalis.

Quercus Esculus

- Ilex

- Robur.

Reseda lutea.

Rhamnus catharticus

Rhamnus saxatilis .

Rosa Pollinii. Spreng. PI. min. cogn. pug. II. p. 66 . Nelle siepi lungo la strada che da Brentino scorge al porto della Pilarola. 
R. trunco aculeato, petiolis aculeato-glandulosis, foliolis ovaso-rotundis, utrinque glabris, serratis, dentibus glanduloso-serrulatis; calycum tubis ovatis, pedunculisque hispido-glandulosis. Pollin.

Pl. nov. vel min. cogu. p. 3 .

Rhus Cotynus.

Salix aurita

- capraea

Salvia glutinosa . Lungo le scale.

- verticillata. Nellá valle di Brentino.

Saponaria ocymoicles.

Satureja montana.

Saxifraga dizoon $\alpha$.

- $-\beta$.

Ponae

tridactylites.

Scabiosa graminifolia.

Scolopendrium officinale.

Scorzonera humilis. (Scorzonera di larga foglia. Pona Bald. ital. 13. 155.)

Scutellaria galericulata. Presso l'Adige.

Sedum album

- reflexum

- - rubens. Mihi.

\section{sexangulare}

Telephium.

Selinum rablense: caulc sulcato ramoso, foliis triternatis laciniis linearibus angustissimis acutis apice diaphanis, involucri universalis foliolis linearibus. Spreng. Pl. min. cogn. pug. II. p. 51. Ferula rablensis. Wulf. in Jacq. coll. 4. p. 312.W. Sp. pl. I. 1412. Setinum elegans. Balb. Stirp. min. cogn. pag. 23. tals. 4 . Ferula alpina humilis, foliorum pinnis angustissimis, trifariam, quinquefariam divisis. Segu. Ver. II. p. 37. Moren. hort. sicc. Seseli massiliense Matth. Counmeut. 750. c. ic Seseli Massiliense di Diosenride. Pona Bald. ital. 212.

Sescli elatum.

Silene Saxifraga.

Solorina saccata.

Sorbus aucuparia.

Spiraea Aruncus.

Sporotrichum aurcum. Linh.

Stachys sylvatica.

Tamarix germanica. Nell'arena lungo I'Adige.

Tamus communis.

Teucrium Chamaedrys

- - $\beta$.

- montanum.

Thesium Linophyllum.

Thymus Acynos

_- angustifolius

_- Calamintha

- lanuginosus

- Nepeta

- Serpillum.

'Tilia europaea

- microphylla.

Tortula tortuosa. Hedw.

Triticum caninum.

Ulmus campestris - $\beta$. latifolia.

Urceolaria calcarea .

Uredo campanulae. In C. petraeae fol.

Valantia glabra.

Valeriana saxatilis

- montana

- tripleris .

Variolaria lactea .

Veronica Teucrium

__ urticaefolia.

Verrucaria epipolaea

- Harrimanni

plumbea

Schraderi

trachona

- umbrina $\beta$. nigrescers.

Viburnum Lantana.

Vitis vinifera. 
Terminata la corsa a monte Baldo ci riducernmo a Brentino, ove passammo la notte. Il dimane ci alzamtno coll'aurora onde far ritorno alla città . Ma per non correre sull' orme altre volte calcate piegammo alquanto il cammin nostro verso meriggio, e arrivammo a Caffi . Quivi visitammo la solitaria casetta dell' elegantissimo scrittore della Sifilide, e salutammo la yaga collinetta, ove solea muovere a diporto, e beavasi della gioconda veduta della sottoposta campagna, e contemplava le bellezze del Benaco e del sublime Baldo. Lasciato Caffi valicammo l'Adige a Pontone. Ecco, diss'io, allora a' compagni, ecco il suolo ov'era nel sedicesimo secolo il rinomato giardino Nichesola. Una diserta iscrizione rammenta a stento al viaggiatore instrutto l'esistenza d'uno de'primi giardini botanici dopo il risorgimento delle lettere. Ove rigogliose vegetavano peregrine stirpi australi, crescono ora sterpi ed ortiche.... La sera fummo in Verona.

Da Verona alli 55 Agosto..... 


\section{AVVERTIMENTO}

Mentre era per escire a luce la presente operetta, il Sig. Giovanni Petrettini da Venezia, il quale come Censore alle stampe ebbe a vederne il manuscritto, invio una cortesissima lettera all' Autore, nella quale fa parola di due valenti Botanici Veronesi, che nel $16 .^{\text {mo }}$ secolo percorsero il monte Baldo, e pubblicarono la storia de' semplici da essoloro scoperti. Poichè non tutti conoscerarno i nomi del Calceolari $e$ del Pona, e a cui fieno noti non increscerà certamente udire da elegante penna l'elogio di due benemeriti antenati, si ̀̀ creduto pregio dell'opera il farla pubblica.

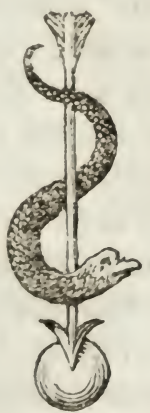

MATVIR T D M 
132

\section{ALL' ILLUSTRE SIGNOR DOTTORE}

\section{CIROPOL L I N I}

Nello scorrere quella sua elegante Operetta, ch'E1la sotto gli auspizii di un dotto Tedesco vuol mettere a stampa, ebbi campo di ammirare più volte le sue vaste cognizioni nelle naturali discipline, e di congratularmi meco stesso, veggendo che in questa nostra Italia, cosi di quelli che alle Umane Lettere rivolgono i loro studii, come degli altri, che alle Dottrine dell'esperienza e dell'osservazione si danno, non è del tutto spento il seme gentile. Piacquemi ancora di scorgere ch'Ella, altrui lasciando la gloria di visitare attentamente l'interne parti dell' Africa o le più alte cime de $^{2}$ monti americani, abbia voluto piuttosto dimostrare i nostri patrii tesori, i quali o non furono mai conosciuti, od a gran torto giacquero dimenticati : e quel suo Montebaldo eziandio, chiamato a buona ragione l'Orto d'Italia, in questi ultimi tempi, ne' quali l'arte de'Semplici va tanto avanti, doveva promuovere le cure di un investigatore diligente. Che se bene esso Monte puó, direi quasi, inorgogliarsi per la copia di peregrini spiriti, che assai prima d'ora andarono con grande amore a ricercarlo, lasciandone ne' loro scritti onorata la rimembranza, non pertanto a' di nostri non ha cosi grande la fama, che a lui si spetta, per colpa forse di quell'avverso destino, il quale fa che i nostri ingegni, i quali le scienze coltivano, 
siano conosciuti si poco: ed anzi bene spesso avviene che un qualche moderno oltramontano si tenga come primo scopritore di tal cosa, di che già noi un secolo innanzi avevamo notizia esatta ed intera. Quindi lo stesso Montelualdo, che ora io veggo nominato di rado, fu dagl'intelletti Veronesi del secolo xv r cosi bene descritto, che, considerata l'età, in cui vissero, non credo che veruno possa desiderare piu oltre.

Giovanni Pona, speziale al Pomo d'oro, in un suo libro trattò di moltissimi semplici di Montebaldo é de' suoi contorni, ed ivi figurò e fece chiare molte peregrine piante degli Autichi, a' Moderni non note. Se non che principe in tale fatica devesi avere Francesco Calzolari, speziale alla Campana d'oro, che nel suo Viaggio di Montebaldo, stampato nelle due lingue d'Italia, ne dichiara il sito e le varie vedute con tanta facilità ed eleganza di stile che veramente a qualunque onorata Compagnia, come scrive egli stesso, a cui per via di diporto o per vedere qualche bella pianta nel suo suolo natio, piaccia di colà trasferirsi, quella sua Guida può servire di ammaestramento non solo, ma di piacevole lezione. E tanto più merta l'Autore di essere conosciuto e letto, essendochè come promotore, anzi ristauratore della Facoltà Erbaria puossi tenere senza dubbio veruno. Stretto da' vincoli della più dolce amicizia col Mattiolo, questi ne' suoi scritti fece del Calzolari frequente menzione, e spesso confessò di avere da esso ritratti assai lumi non solo nella Botanica, ma ancora nelle Scienze mediche. Il Calzolari ebbe molte altre ed insigni corrispondenze, e singolarmente con alcuni eruditi Viaggiatori, che a raccogliere le esotiche piante si recavano per le terre 
più inospitali e remote. Ricorderò soltanto Cecchino Martinelli veneziano, che ad esso spedi da Damasco il Satyrion, pianta, che allora credevasi atta a risvegliare le voglie di Venere, e di Haimo Abensusio, ebreo ed archiatro dell'Imperatore de' Turchi, che gli mandò l'Amomo e più altre cose. Fu altresi famigliare di Ulisse Aldrovandi, che forse lo confortò a dar principio a quel suo tanto famoso Museo, che venne poi in luce a' tempi di suo nipote, pure per nome Francesco, e che con grande apparato di dottrina fu illustrato dal Ceruti e dal Coccio. Nè solo dagli scienziati, ma fu avuto in sommo pregio anche da' più gran Principi italiani e stranieri. L'Imperatore austriaco Massimiliano voleva avere di sua composizione i più valevoli contravveleni, e Vincenzo Gonzaga, gran fautore e cultore egli stesso de' buoni studii, così l' ebbe in istima che d'un'aurea collana volle onorarlo. E di più ancora degnossi di andare a visitarlo in un'amena sua villa vicino a Verona, nè si die' a credere di menomare la sua dignità, sedendo alla mensa dello Speziale Calzolari, quando questi, già d'anni carco e cieco degli occhi, attendeva alle predilette sue occupazioni. Tale si è quell' uomo a gran torto dimenticato, che in società di Antonío Tolomei, di Girolamo Lippomani, di Lodovico Fumanello, di Luca Ghino e del Fracastoro si dilettava di ricercare in ogni parte l'amato suo Monte, e che avendone fatta una Descrizione volle mandarla in dono a Prospero Borgarucci, perchè avesse luogo nella Fabbrica degli speziali, che questo Autore per i torchi del Valgrisio nel 156 ? fece di pubblico diritto: dono, cui l'uomo insigne e viaggiatore perito ebbe si caro, che nel fine della sua 
Fabbrica gli diede nobilissimo luogo, siccome compimento il più bello che potess'egli offerire a' risguardanti .

Ond'è che io stesso, sapendo, signor Dottore, il di Lej ottimo divisamento di pubblicare quell' ameno suo Viaggio, le invio la notizia di questa Operetta, perch'Ella ne faccia quell' uso che più crede opportuno, e considerarla le piaccia non già come compimento del suo bel Libro, ma come scritto rozzo ed informe, cui le moltiplici occupazioni non mi lasciano tempo di pulire, e di ordinare. Nè io dettandolo ebbi altro scopo fuorchè quello di dimostrare in qualche piccola parte che gl'Italiani furono sempre teneri cultori anche delle cose appartenenti all'Istoria Naturale, e che anzi eglino siccome nelle più soavi e mansuete discipline, cosi pure in queste devono per il tempo decorso avere il primato. Dell' età nostra non so quale le venture genti pronunzieranno sincero giudizio, ma so bene per altro ch'Ella, se colle Opere sue continua a mantenere di tali studii viva la fiamma, offerirà bell'argomento alla Letteraria Istoria.

Di Venezia 9 Ottobre 18г6.

\section{Umilissimo Servitore}

Gionanin Petretinis. 


\section{O N T E N U T O}

NELLA LETTERA I.ma SUL IAGO DI GARDA

\section{Partenza da Verona per Bardolino (c.te 4 ). De-} scrizione del Lago di Garda o Benaco, sua lunghez$z a$, larghezza, profondità, ed elevazione della sua superficie sopra quella dell' Adriatico ( 4-5). Innalzamento delle sue acque in estate (5). Sue tre isole (5). Penisola Sermione (5). Sarca, Ponale, Tusculano, ed altri torrenti che metton nel Lago (5). Mincio che n' esce (5). Suoi due venti periodici (5-6). Sua navigazione (5). Purezza delle sue acque (6). Amenità e varietà de' suoi contorni (6). Sponda Veronese. Garda, sua Rocca ed Eremo (7). Promontorio di S. Vigilio, il pii ameno luogo della sponda Veronese (7). Torri, Castelletto, Brenzone, Malcesine (7). Sponda settentrionale. Torbole e Riva (8). Sponda occidentale piu deliziosa della Veronese. Cateratte del Ponale (8): Limone, Campione (8). Ridente seno di Gargnano (8). Tusculano, sue cartiere, e fucine (8). Digressione sull' esistenza dell' antica città Benaco (9). Due iscrizioni antiche (9). Maderno (10). Salò e sua gaja riviera (10). Manerbe, Desenzano, Rivoltella, Sermione e grotte di Catullo, Peschiera, Lazise (10). Struttura geologica delle sponde (10). Sponda Veronese parte calcare, parte di terza formazione (10. II) Marmi eleganti di Tor- 
ri (10). Ciotti porfirici e granitici ond'è sparsa lı sponda (10). Sponda settentrionale e occidentale camposte di calcare secondaria bianca o rossiccia, o nericcia fetente, o di marna calcare con corpi organizzati marini petrefatti (I I ). Varj colli Salodiani di terza formazione (xI). Palle silicee e marnose racchiuse nella calce fetida di Campione ( I I ). Marmo nero di Tremosine (Ir). Cristalli di quarzo, ciottoli di diaspro, calcedonie, agate, corniole, petroselci erratici sui colli di Salò ( I r. I22). Arena della spiaggia composta di ciottoli calcari, granitici, porfirici, schistosi ( 12 ). Cenno sulla costruzione degl' interni monti Bresciani ( 12 ). Miniere di ferro ivi esistenti (12). Torba della sponda meridionale (12). Acque termali gorgoglianti dal Lago presso Sermione (12). Corrente singolare subacquea (13). Sua cagione ( 13. 14). Indice alfabetico dei vegetabili principali crescenti presso le sponde e nell' acque ( I4-20). Piante particolarmente menzionate. Buphtalmum speciosissimum (15); Erythraea Centaurium; Erythraea intermedia (I6); Favolus Mori (16). Nota sul genere Hexagonia dell'Autore (16). Senecio aquaticus (r9); Senecio Jacobaea (r9); Ulva turbinata (20), e sua descrizione (44. 45). Pesci lienacensi indicati coi loro nomi scientifici e volgari (20.21). Descrizione di due specie nuove chiamate Blennius vulgaris, Cyprinus benacensis colla loro fisura di grandezza naturale disegnata dall'autore (20.21.22). Petromyzon marinus, e Plexronectes flesus alitatori dei fumi Veronesi (20.22). Tre Granchj del Benaco (22.23). Augelli che frequentano il Lago coi proprj nomi scientifici, italiani, e volgari 
(23. 24). Principali augelli che vivono o passano per la provincia Veronese (24-28). Insetti rari od eleganti (29-32). Descrizione di un Bisso dall' autore chiamato Dematium coleopterum, vivente sui coleotteri(29). Nota intorno alla Caprificazione (3г.32). Sei serpenti della provincia veronese (32-34). Con-siderazione sulla direzione dei rami inferiori degli alberi crescenti sul pendio de' monti (35). Osservazioni mi-croscopiche e sperienze eseguite sulle Conferve (36.37), Oscillarie (37.38.39.40.41), Linchie (4r.42.43). Specie di Linchia, Linkia pulposa, annoverata dai Botanici fra' Licheni (43).

\section{Lettera II. SUL MoNte Baldo.}

Partenza da Bardolino per Caprino (47). Posizione di monte Baldo ( 47 ). Sua altezza, lunghez$z a$, larghezza (47). Sue vedute, amenità, e allettamenti ( 47.48.49). Arcobaleno osservato dall' autore sulle cime di monte Baldo sotto figura di circolo (49). Lunghezza, larghezza, e superficie quadrata della provincia Veronese (50). Sua pianura, suoi monti, fiumi, e torrenti(5o). Longitudine, e latitudine di Verona (5o). Sua distanza dall'Adriatico (5o). Sua altezza dal livello dell' Adriatico (5o). Altezza di molte cime e luoghi de' monti Baldo (5r.52), Bolca (52), Lessini (52), Sumano (52.53), del lago di Garda(5r), e di pii altri siti (5I-53). Monte Baldo e rimanenti monti Veronesi diramazione dell'alpi primitive del Tirolo (49). Confini e valli principali de' monti Veronesi (5r:52.53/. Letto dell Adige cambiato e abbassatosi dopo lo spaccamento de' monti della Chiusa 
(53.54). Monti piic alti del Veronese (54); ignudo rupi per molta parte dell'anno nevose (54); monti minori un tempo boschi ora pascoli (54); composti tutti di calcare stratificata con filoni e straterelli di creta, marna, argilla, silice, e zeppa di vegetabili e animali petrefatti (54.55). Ittioliti innumerevoli di m. Bolca (55). Osteoliti (56). Ossa e zanne d'elefante e d'altri quadrupedi del Serbaro (56). Marmi pregevoli e numerosi del Veronese (56). Oolite e calcare in bastoni di m. Baldo (57). Tartufite di Castagnè (57). Spelonche (57). Ponte di Veja (57. 58). Roccia base de' m. Veronesi in niun luogo visibile, bensi nelle prossime provincie (58). Sono lo schisto micaceo e l'argilloso sovente coperti da un' arenaria macigno (53). Trappo vulcanico comunissimo ne' monti Veronesi ad ogni altezza (58.59.6o). Monte Tomba il più elevato luogo ove si rinviene il basalto, aggiunte in nota le piante alpine ivi crescenti (60). Vachia e tufo vulcanico coperti dalla roccia calcare c sovente disposti a strati zeppi di petrificazioni, alternanti con istrati calcari (60) . Caratteri della vachia Veronese (60.6r). Mandoloide (6r). Mesotipi bellissime vetrose e farinose di m. Baldo presso Tierno(6r); meno pregevoli in altri luoghi(6r). Stronziana solfatica nella mandoloide di Montecchio (6r). Corpi organizzati marini di $m$. Viale coperti e penetratidalla stronziana solfatica (6r.62). Vachia, tufo vulcanico e basaltino conformati in palle o cipolloni (62). Caratteri del basalto Veronese (62). Basalto amorfo, basalto colonnare (63). Basalto colonnare dellavalle di Roncà, della valle degli Stanghellini $e$ di m. Bolca (63). Prismi basaltini singolari e basalto 
140

in tavole di Trissino (63). Terre gialle e rosse Veronesi (63). Terra verde o talco zografico di $\mathrm{m}$. Baldo (63). Sua analisi e giacitura (64). Talco zografico di m. Bolca e d'altri luoghi (64). Manganese metalloide di Alcenago (64). Miniera di ferro della valle di Botte e della Ferrara, e ferro ossidato lenticolare di Monmaore di Baldo (64) . Ferro solforato frequente sui colli (64). Arenaria di Mazzurega, di Lavagno, e della valle de' Prusti (64. 65). Soda nativa delle mura di Verona (65). Solfato di magnesia erratico nelle cave di marmo di Castione in m. Baldo (65). Muriato di soda di Rovere di Velo, e non solfato come per errore sta scritto nel testo (65). Gesso di Torri (65). Creta di Marcelise e S. Floriano (65). Pietre focaje del Cerro (65). Acqua minerale fredda della valle di Botte in m. Baldo non analizzata (65), e di Rovere di Velo imperfettumente analizzata (65). Acqua termale di Domejara recentemente scoperta (66). Sua profondità singolare (66), sua posizione (66) ed elevazione sopra l'Adriatico (66). Caratteri fisici, e peso specifico $(66.67)$. Analisi instituita dall' autore (66. 67. 68). Acqua termale di Caldiero (69) . Calore, peso specifico ed analisi (6g). Litantrace o carbone fossit di piu fatte copioso in varj luoghi della provincia (69.70.71). Carbon fossile di m. Bolcus sourapposto agli ittioliti e sottoposto al basalto colonnare, e coperto e intersecato dal trappo ( 70 ). Tufo calcare, breccie, e puddinghe component $i$ molt $i$ colli e monti meno elevati (75). Alta pianura Veronese costituita di banchi di sassi di varie specie strascináti dall' Adige e da' torrenti ( $7 \mathrm{I}$ ). Monte Baldo povero in ogni classe d'animali dagl'insetti in 
fuori, feracissimo all' opposito in piante $\left(7_{2}\right)$. Vie da $V e-$ rona al m. Balilo (72.75). Topografia di m. Balilo $\left(7^{2} \cdot 7^{3} \cdot 74 \cdot 7^{5} \cdot 7^{6}\right)$. Brentino villaggio a' piedi del monte (72). Santuario della Corona (73). Coltri e la Croce o Crosati (73). Il Prabasar(73). Valle Basiana (73). Valle fredda (73). Sassi di valle-fredda $\left(7^{3} 5\right)$. Valle Ime (73). Il Campedello (73). La Ferrara villaggio (73). Cimndetta l'Albarè (73). Valle Orza (73). Il Cumbrigar (73). Valle Novesa (73.74) . Torrente Aviana (74.75). Il Campione (74). Le Acque-negre (74). La Lonza (74). Valle brutta (74). Valle Losanna (74). Il Sassetto e sua fontana (74). Monte Gambon (74). Il Cerbiolo (74) . I Lavaci (74). Piano della cenere (74). Selva d'Avio (74). Valle dell'Artillon (74). I Dossioli (74). Prati di-Brentonico e del Tredespin (74). Selva de' Brentegani, rammentata dal Pona, distrutta (74). Gli Zocchi (74. 76). Navene (74). Bocca di Navene d'onde si scende al Benaco (76). Tolghe (74.76). Canalette $(74 \cdot 76)$. Altissimo $(74 \cdot 76)$. Le Scalette(74). I Pianetti (74). Pozza Ferrera (74). S. Giagomo (74).Villaggi Brentonico, Castione, Tierno a settentrione (74. 75$)$.Valle e torrente Sorna (75). Il Tretto (75). Sentiero de' soppiadori per cui si scende al villaggio Avio alle falde orientali (74). Via che da Caprino conduce ai monti della Corona, detta la Costa dei cani (75). Altra vic detta dei Lumini che conduce a monte Piore e allcs valle Ortigara (75). Prati e selve di Pra-lungo e di Brenzone (75). Vetta detta Costa-bella (75). Valletta di Navole (75). Fontana di Navole ( $\left.7^{5}\right)$. Valle del Bastion (75). Valle Vaccaria (75). Fonte di Brigaldello o delle Buse (75). Selva di Piombe (75). MIonmaor la più alta cima di m.Baldo $(75)$. Coval santo 
142

(76). Valli profonde ed aspre delle cime di monte Baldo, chiamate valle delle buse, delle pietre o di monmaor, delle ossa, di S.Zeno, grande o fonda o dritta, Orzera, Finestra, delle Pozzette (76). Colma o cima, prato, e selva di Malcesine (76). Orrido sentiero detto di Ventrar che scorge al prato di Malcesine $e$ al Benaco (76). Geografia botanica Veronese, Vicentina e Bresciana (77), divisa in tre regioni o zo ne (77). Instabilità de' confini delle tre regioni nel variar della plaga (77), comprovata per molti esempi $\left(77^{\prime} \cdot 7^{8)}\right.$. Cagioni che determinano l'abitazione delle piante ( $\left.7^{8}\right)$. Enumerazione alfabetica delle piante rinvenute dal$l$ ' autore in m. Baldo e ne' luoghi adjacenti, nelle molte gite da esso intraprese, coll' indicazione dei siti e delle loro altezze sopra l'Adriatico (79-129). Piante rinvenute nella valle di Caprino e ne' luoghi adjacenti alle vie onde si ascende in $m$. Baldo fino ai Masi $e$ al Pra-bestemnià all'altezza di yoo metri (79.--93). Dai luoghi suddetti fino alla valle Ortigara all' altezza di 1450 metri( 93-97). In Costa bella; 1450 ai $2000 \mathrm{~m}$. (97-99). In valle Vaccaria e al fonte di Brigaldello, тү00 ai г900 m. (99-гоo). Nella valle di Monmaor e lungo il sentiero onde si ascende alla cima, 1800 ai $2200 \mathrm{~m}$.(100-101). Ai Coval santo, Monmaor, Sascaga, Sassetto sino al di là della colma di valle Finestra; 2000 ai 2200 e più m. (10I-102). Nelle valli delle ossa, di S.Zeno, grande o dritta, Orzera, Finestra, I700 ai $2200 \mathrm{~m}$. (102-105). Ai pascoli e selve dei Zocchi e Tredespin, e alla colma e al pra di Malcesine, 1400 ai r600 m. (го6). Selva di Malcesine, 200 ai $1300 \mathrm{~m}$. (10-). Al sentiero di Ventrar, $1700 \mathrm{~m}$. (107). Alla bocca di Navene e nelle 
prossime selve lungo la via per cui si scende al Benaco, 1300 ai $1600 \mathrm{~m}$. (108). Al Lastì oltre Navene e in Altissimo, 1600 ai 2100 e piu m. (108). Dalle falde settentrionali verso Tierno, Castione, Brentonico, valle delle Sorne, ascendendo per S. Giacono, Pozza-ferrera, fin' ai Pianetti, 300 ai $1000 \mathrm{~m}$. (109. I10). In Canalette, Tolghe, valle del Tretto, iungo il sentiero de' soppiadori, I000 ai $1200 \mathrm{~m}$.(II0). Al prato di Brentonico, I3oo. m. (I I o-I I I). Nella valle dell'Artillon, I300 ai г500 m. (II . I12). Alle Acquenegre e al Campion, 1300 ai r500 m. ( I12-113). Alla via dei Lavaci e al Pian della Cenere, dai 1000 ai r $400 \mathrm{~m}$. (г13-rr4). Nella selva d' Avio e lungo la via per cui si discende al villaggio, dai I5o ai $900 \mathrm{~m}$. (I14). Ai pascoli del Cerbiol e di m. Gambon, I3०o ai I $400 \mathrm{~m}$. (1 14). In Novesa, I500 m. (Ir4. I I.7). INella valle Losanna, 1500 ai $2000 \mathrm{~m}$. (I I ) . Alla Ferrara, 800 ai 1000 m. (I17. 120). Alla Lonza, Campedello, valle brutta, 1200 ai $1300 \mathrm{~m}$. ( 120). Nella vallefredda e sue rupi fino alla valle di Navole, I 400 ai I $650 \mathrm{~m}$. (120. 122). Alla fontana di Navole e alla valle del Bastion, I700 m. (г22). Nelle valli Basiana $e$ Ime, 1200 ai $1300 \mathrm{~m}$. ( 122. I23). Ai prati del Prabasar e della Croce o C'rosati, 1000 ai $1100 \mathrm{~m}$.(123). Dai Coltri al Santuario della Corona, lungo le scale, selve e rupi laterali sino alla valle di Brentino, 150 ai $900 \mathrm{~m}$. (123-129). Nota sulla virtù della radice della Saginella selvatica o Andropogon halepensis, sperimentata dall' autore in varie malattie (124). Ritorno da Brentino a Verona (130). Visita alla casetta del Fracastoro presso Caffi (13o). Si valica l'Alige a Pon-tone (r3o). Luogo ove esisteva nel secolo decimo sesto il 
giardino botanico Nichesola (r3o). Arrivo in Verona (130).

Lettera del Sig. Petrettini all' Autore, nella quale è ragionamento de' due Botanici Veronesi Calceolari $e$ Pona, che nel secolo $16 .^{\text {mo }}$ intrapresero il viaggio di monte Baldo $c{ }^{\text {to }} \mathbf{1} 32$.

Piante nuove o rare o diluginate e officinali ED ECONOMIGHE I'IU' PREGEVOLI.

Acer monspessulanum. c. ${ }^{\text {te }}$ I 14 .

A. platanoides . ir 4.124. A. Pseudo-Platanus. II 4 . 124.

Achillea ambigua. II?.

A. atrata. 101. 102.

A. Clavennae. 60.97. гог. A. distans . $9^{3}$. I I · $\beta$. I I 7 . A. nobilis. 120 .

Aconitum Anthora. 120. A. cernuum . 93. 120.

A. cernuum var. $f$ l. albovariegato. I $\mathrm{I}$. II I. A. Lycoctonum . 93. III. $\mathrm{I} 20$.

A. Napellus . 93. 111. 120. Actaea spicata . 93. 124. Adonis annua di cui sono varietà l'Adonis aestivalis e l'A. autumnalis. 79. Agaricus edulis. 79. A.flammeolus. I I I. II3. Agave americana. I4. Asrimonia Eupatorium . I4. 79 .

Aira montana. so8. Ajuga Camaepithis . 79.
Ajuga piramidalis . 100. A. reptans. 79 . II?. Alchemilla alpina . 97. IOI. I20. Alisma Plantago. 14. Allium neapolitanum. I4. A. ochroleucum . II 7 . A. paniculatum $\beta$. bulbiferum. I 2 I. Althaea hirsuta. II4. A. officinalis. I4. Alyssum rupestre. I02. 107. J20. 124. A. satioum . 79 .

Amaranthus retroflexus. I4. 79 .

A. sylvestris. I4. 79.

Anagallis arvensis. $2 . \beta .79$. A. tenella. 14. Anchusa officinalis 80. Anemone alpina. 60. I0I. $A$. baldensis . I02. Andropogon halepensis. 14. 80. 124. Androsace alpina. ror. A. lactea. IOI. Anethum Foeniculus. 1480. Angelica syluestris.80.124. 
Angelica syluestris (3. montana. 99. ro?.

Anthemis alpina. 102.107. A. tinctoria. 14.80.

Anthyllis montana. 99.

Apargia alpina . 93.

A. crocea . IоI.

A. incana. 14. 124.

Arabis alpina. 93. II2.

$114.11 \%$.

A. nutans. $10 \mathrm{r}$.

A. saxatilis. I0I.

Arbutus alpina. 102. 107. A. Uva Ursi. 107.120.

Arctium Lappa. 80.

Arenaria bavarica.14. 110.

I20. 124 .

A. ciliata.97. 10 I, 107. 120.

A. Gerardi, di cui dansi

tre varietà .93. 102. 103. 120.

A. polygonoides. IC 3 .

A. striata. 60. 107.

A. tenuifolia $\alpha$. decandra e $\beta$. pentandra. 80.

A. trinervia . I 3 .

Aristolochia rotunda. II4.

\section{2.}

Armeria vulgaris. Iо3.

Arnica montana . 94. 121. 123.

A. scorpioides, di cui sono varietà l'Arnica glacialis e l'Arnica Wulfeniana o cordata. 103. I 17 .

Arthemisia Absynthium.14. 80.

A. campliorata, di cui è varietà l' $A$. subcanescens. 14.30.
Arum italicum. 14.30.

A. maculatum . sog.

A. maculatum var. sine maculis. II4. 123.

Arundo Donax. 14.

A. Phragmites. I4.

A. pygmaea. 104 .

Asarum europaeum. 105.

Asparagus acutifolizss. I4. 80.

Asperula odorata.III.II4.

A. taurina. III. II 4 .

Aspidium alpinum. 103.

A. Halleri. I 18 .

A. Pontederae, varietà dell' Aspidiun fragile . I 03 . I 17 .

A. rigidum . 104 .

Asplenium viride. го8. п3. Aster alpinus. 122. A. Amellus . 14. 80. Astragalus Clusii. 123. A. montanus. 97. ror. A. Onobrychis. 80. Astrantia major. II?. Athamantha Cervaria. I4. A. retensis . 100. I01. 104. 107. 12 I. 125. Atragene alpina. 94. 99. I07.

Atropa Belladonna. 14.94. I 2.

Azalea procumbens. 100. Barbula inclinata. 122. Bartsia alpina . 100. I0I. Betonica alopecuros. 94. I 06 . III.

B. hirsuta. 106. III. II4. B. officinalis. $14.113 . \beta$. fl. aibo. 118 . Bidens bipinnata. I4. 80. 
Boletus edulis. 8o.

Borrera chrysophthalma. $8 \mathrm{x}$.

Botrychyum Lunaria. I 3 .

I 2 I.

Bryonia dioica $.8 \mathbf{x}$.

Buphtalmum speciosissimiem. s 5 .

Bupletırum baldense . 125 .

B. graminifolium . I0I.

B. ranunculoides . I 25 .

Campanula bononiensis .

15. 106.

C. petraea. $\mathbf{1} 25$.

C. rotundifolia $\beta . C . p u-$ silla. I I 7 .

C. spicata. 125. et $\beta$. fl. albo .125.

Carduus crassifolius. II5.

C. montosus. I 5 .

C. sumanus . 216 .

Carex baldensis. I5. III.

II7. I20. 12 I. I 22.

C. capillaris.99. IOI. I2I.

C. firma. I O I

C. Michelii. 8r.

C. ovalis. 101.

C. praecox. $8 \mathrm{I}$.

Carlina acaulis . $8 \mathbf{r}$.

Carum Carvi. 94. I18.

Castanea vulgaris . 8I.

Cenonyce vermicularis $\beta$. taurica. I0 - 108.

Centaurea Jacea $\beta . C . a-$ mara. I5.8г. I I8.

C. Rhapuntica. 108. I2I. rerastium latifolium . 60. ro4. $12 \mathrm{I}$.

Cercis Siliquastrum. 15. 82.

Coterach officinarum. I 13 .
Cetraria cucullata $\beta$. nipharga. 108.

C. islandica . 97. 107.

Chara flexilis $\alpha \cdot \beta .82$.

Cherleria sedoides. 60. 97. Ioo.

Chrysanthemum Leucanthemum, di cui sono varietà il C. atratum e il $C$. montanum.82. II 7. 12 I. Cineraria alpina, di cui costituiscono la varietà a. la C. alpina $\beta$. Linnaei Sp.pl.o Senecio alpinus dello stesso suppl. $e$ del $W . S p . p l . e$ del Persoon; la varietà $\beta$. la Cineraria cordifolia Linnaei Suppl. e W. Sp. pl. et Persoon o Cineraria alpina a. Linnaei $S p$. $p l$.; la varietà $\gamma$. il Senecio baldensis Encyclop. botan. et Persoon. 94. Circaea alpina. I12. I16. Cnicus erysithales fl. luteolo et fl. purpureo. 107.

Cnicus rivularis . 106. 108. Comarum fragarioides. 122.

Convallaria majalis. 112. C. multiflora , 123.

Convolvulus Cantabrica. I5. 83 .

Cornus mascula. I5. C. sanguinea 83 .

Coronilla Emerus . I 5 .

C. minima. 83.

Corylus Avellana. I5. 83. Crataegus vel Mespilus monogyna. I5. 87. 
Crataegus Oxyacantha.87. Cyclamen europacum . I5. Cucubalus Behen ß.montanus. I2I.

Cyperus glomeratus $W$. Enum. 15. rog.

Cypripedium Calceolus. I07.

Cytisus alpinus . 93. 106. C. argenteus. 16 .

C. Laburnum. 93.122. 125. C. purpureus.i6. rog. II 4 . Dapine alpina . $\mathrm{r} 6$.

D. Cneorum . 97. 12r.

D. Laureola . i6. Ir4.

Dematium coleopterum.29. D. Mezereum.93. п12. 114. Dentaria bulbifera . I12. Dianthus Armęrius . 93. D. atrorubens . 16.84 .

D. Carthusianorum . I6. 84. 109.

D. plumosus . 16.101. 109. I 21 . 125.

$D$. prolifer. 84.

D. syluestris . 15.84. 100. Dictamnus albus . 16.84 . I09. 126.

Digitalis ambigua . 84. $9^{5}$. I I2. 121 .

D. lutea. 84. I12. 126. Doronicum austriacum . I12. 116 .

Draba aizoides. 97. I0I. I $2 \mathrm{I}$.

D. pyrenaica . I0 I.

D. verna. 84.

Ectosperma baldensis.122. Dryas octopetala . 60. 97. IOI.

Epipactis Nidus Avis.113.
Erygeron alpinum . 97 . Erysimum hieracifolizum. 126.

Erythraea Centaurium.i6. E. intermedia . I 6.

Euplorlia ambigua. I16. 121.

E. Lathyris . 16.

E. maculata . 126.

E. nicneensis. 84. 109.

Euphrasia tricuspidata . 16. 109. 120. 126 .

Evernia divaricata. гог.

Evonimus latifolius. 16 . 107.109.

Favolus mori ( Hexagonia mori. Pollin.) col suo carattere generico e specifico . I6. 126. 127.

Ferula nodiflora . 17· 127. Festuca ciliata. 84. $F$. serotina . 17. 84. 109 . Ficus Carica. I ?

Fragaria collina. 84.

Fumaria capreolata . I 7 .

F. officinalisa. 3.84.

Galeopsis cannabina. II8.

$G$. grandiflora. I 18 .

Galium baldense.60.97.100. Genista pratensis. 118.

Gentiancs ciliata . 125.

G.lutea.99.110.113.121. Geranium argenteum. 98.

IOI.

G. macrorrtizon - 104 .

Geum montanum. 60. 98. 102. 108. 3. 102.

$G$. rivale. 118 .

G. urbanum. 85. 127 .

Globularia nudicaulis.1 I ?. 120. $121,127$. 
Gnaphalium dium . 98.

Gratiola officinalis. 17 .

Grimmia ovata 85.

Hedysarum Onobrychis 85.

Heiianthemum Fumana . 127.

H. grandiflorum . 98. I2I. H. marifolium, di cui costituiscono la varietà $\alpha$. il Cistus marifolius e ilC. canus, e la varietà $\beta$. il Cistus anglicus. 85. 12\%. $H$. oelandicum, di cui costituisce la varietà $\alpha$. il Cistus oelandicus, e la var. $\beta$.il Cistus serpillifolius. 98. 121 .

H. salicifolium. 85.

H. Seguieri . 98 .

Helleborus foetiduis.17. 85 . $H$. niger. I 7 .

H. viridis . 17.85.

Helvella leucophaea. 85. Hemerocallis Liliastrum. I 18 .

Heracleum flavescens. II8. H. pyrenaicum, forse l' $H$. gummiferum del Willdenow, che somministra la gomma ammoniaca. 104. Hesperis tristis . 17 .

Hieracium aureum. 102. H. chondrilloides . 102. Hydnum coralloides. 86. Hydrodiction pentagonum. I 7 .

Hyosceris foetida . 66 . Hypericum Androsaemum. I 3 .
Hyperisum humifusum.1 7 . Hypochaeris helvetica.106. Hypocrepis comosa . 127. Iberisrotundifolia.102.104. Ilex Aquifolisum. 17. I I4. var. inermis . 17 .

Impatiens Noli tangere. II2.

Iris Pseud-Acorus . I7.

I. sambucina. $\mathbf{1 2 7}$.

Tuncus trifidus. 60. 102. Jungermannia pinguis . 127.

Juniperus nana. 99. 104. II2. I 2 I.

Lactuca perennis . I $77^{\circ} 36$. L. Scariola. I 7. 86.

Lamium Orvala . I I 9. 127. Laserpitium peucedanoides. 99. 121. 122.

L. Siler. I 7.127 .

Lathyrus setifolius . 86.

L. sphoericus. 86.

Laurus nobilis. I7?

Lavandula Spica. I7.

Lecidea baldensis .86. Iog. 127.

Leersia oryzoides. I 7 .

Lepidium alpinum . 102. ro4.

L. petraeurn. I7. I02.

Ligusticum peloponense. iा 3 . ri 6 .

Lilium bulbiferum $\cdot 9^{6}$.

L. Martogon - 96. 119 .

Limodorum abortivum.127. Linkia pulposa. I7. 86 . I09. I2?.

L. verrucosa. I7.

Linaria alpina. 104.

Linum alpinum. I2 . 
L. viscosum . 119.

Lonicera alpigena: 96 .

Lunaria rediviva. I I4.

Lychnis quadridentata. 60. 104.107.

Lycoperdon gisanteum. II6.

Lycopodium selaginoides . 60. 102.

Lycopus exaltatus. 17. 86. Lythrum Salicaria. 17.86. Malva Morenii . 1ng. 123. Miarsilea quadrifolia. I 7 . Melilotus officinalis $\alpha . \beta$.

17. 87 .

Melissa officinalis . I ? 28. 87.

Melittis Melissophyllum. 17.87 .128 .

Mentha Pulegium . I 7 .

Mörhingia muscosa ${ }_{128 .}$ Muscari botryoides. Iro. Myagrum striatum . 87. Nepeta nuda . 17. 96. 122. Nerium Oleander . 18.

Olea europaea. II 8.

Ononis minutissima. 88. IIO. I28.

Ophrys alpina. 6r 98.99. I2 I .

O. apifera . 8.88.

0 . arachnites . 18.88.

O. aranifera. 18. 88.

O. Monorchis. I I3. I20.

Orchis albida. I 13 .

O. bifolia I 13 . п16.

O. conopsea. 06г. I19. 128.

O. fusca. iाо.

O. globosa. гов. I I9.

O. hircina. I\%. I10.

O. maculata. 123 .
Orchis mascula. n3. I19.

O. Morio. 18.88. ing.

O. nigra. 98. 108. 122.

Orchis odoratissima . 99 . I22.

O. piramidalis.18. 88. I19.

O. sambucina . I2I.

O. sambucina fl. incarnato. I2I.

O. ustuluta. 88.

O. viridis. 108. 121.

Origanum vulgare. 18.88. Ornithogalum luteum. Ir4. Orobus lateus. Ir6. I2 I. Oxalis Acetosella.i I2. I 6. Paederota Bonarotta . 60. 100. 104. 107. 121. 128. Paeonia corallina. 96. I19. 128.

P. officinalis . 96. I 19 . Papaver Argemone. 88. P. aurantiacum. 104. Paris quadrifolia . I12. Pastinaca sativa. 18. 38. Pedicularis acaulis. 106. P.comosa.19. I19. I21. 128. I8. P. palustris . 18.

P. rostrata. 60. 100. 102. P. rostrata var. fl. carneo. 102.

P. tuberosa.100. 102.108. I2I.

$P$. verticillata. 108 .

Peplis Portula. I21. Peziza lycoperdoides. 88. Phallus impudicus . 112. Philadelphus coronarius. 128.

Phillyrea latifolia . 18. Phyteuma comosa.60.102. I05. I2I, 128. 
Phytolacca decandra. 18. Prunus Avium . 89: r14. Pimpinella dissecta. I2I. Pulmonaria angustifolia. I28.

Pinguicula flavescens. I02. Ir3. 12 .

Pinus Abies . ro?.

$\boldsymbol{P}$. baldensis varietà del $\boldsymbol{P}$. Abies. 107.

P. Larix. I07.

P. Picea. II

P. Pumilia . 6o. 100. 105.

I 2 .

Pistacia Terebinthus: 18. 88.

Poa aquatica var. prolifera. I 8.

Polygala amara.116. I I * 122.

P. Chamaebuxus var. $f$. elegantissimo. 100.

Polygonism Bistorta. 96. I 9 .

P. Hydropiper. 18.89. Polypodium Phegopteris. I 13.

Potentilla caulescens . $\mathrm{r} 8$. Poterium Sanguisorba. 89 . Potentilla nitida. I00. 102. 105.

Prenanthes purpurea . I08. x28.

$P$. purpurea var. fol. angustioribus . 108.

Primula Auricula . 100. I02. I 2 I.

$P$. carniolica.60. 100, 102. I2I.

$P$. veris . 18.

Prolifera parasitica. 128. Prunella vulgaris et var. ß. $\gamma .89$.

43.

P. officinalis : 89 .

Pulsatilla pratensis. 89.

Punica Granatum . 8.89 .

Pyretrum elegans. II 7 .

Pyrus Malus. Ir4.

P.Pyraster. II 4 .

Quercus Aegylops . 89.

Q. Ilex . 18. 128.

Q. pedunculata . 18.89 .

Ranunculus alpestris . 98 . 1000. 102. 105.

R. auricomus . 100. 105.

R. rutaefolius.98. 1(10. 105 .

R.Seguieri. 105.

R. Thora a. ß. 100. 105.

Reseda Phyteuma . 18.89. Rhamnus Catharticus.128. R. Frangula. 18 .

R. pumilus.18. 105. 12 I.

R. saxatilis . 18.

Rhododendron Chamaecistus. 102. 105.

R. ferrugineum . 6o. 105. 108. 1 13.

R. hirsutum . I01. 108 . II3. I2I.

Rhus Cotynus . 18. 89.

Ribes petraeum. Ir4.

R. Uva crispa. I 14. I 19 .

Riccia fluitans. 18.

Rosa alpina. 105. 108. 112.

R. Pollinii. 129.

R. pumila. 39 .

R. rubiginosa fl. albo. 18 . 89 .

Rosmarinus officinalis. I8. Rubus coesius. 89. R. hirtus . so8. 
Rubus Idaeus.96. I12. 122. Sedum atratum. I19. 120. R. saxatilis.96. 102. 121 . Rumex aquaticus. I3. R. scut atus. 120.

Ruscus aculeatus . I 8.89 . R. Hypoglossum . 121 . Ruta chalepensis. 18.89. Saccharum Ravennae. 18. Salix Pontederana. I 3 . Salvinia natans. $\mathrm{x} 8$.

Sambucus racemosa. 108 .

\section{I I I I 2 .}

Saponaria ocymoides . $9^{6 .}$ S. officinalis. I8,

Satureja hortensis. 18.89. S.montana.19.90.1 10.129. Saxifraga Aizoon «. $\beta .9^{8}$. 105. 121.129.

S. nndrosacea. I00. 105.

S. bryoides. 102. 105.

S. burseriana . 105 .

S. coesia. 98. 102. 105. 108. $12 \mathrm{I}$.

S. exilis . I12. 122.

S. longifolia . 108 .

S. muscoides. 102.

S. mututa. 60. 105. 108 .

S. Ponae a. 60. $\gamma$. 100. 105. 108. 12 I. 129.

S. sedoides. 105. 108. 11 7 . Scabiosa graninifolia. I9. 129.

S. Succisa. 19. I19. 122.

S. transiluanica . 19 .

Scirpus Caricis . I9.

$S$. palustris $\alpha$. $\beta .1$ I 0 .

Scorzonera alpina. 100.

S. humilis. 129 .

S. rosea. I2 I.

Scrophularia verna. I 3 . Sedum acre. II 9 .

122.

S. rubens. I I9.

S. Telephium. 129.

Selinum rallense. r 29.

S.Seguieri.106.108. I I0.I I Sempervivum tectorum. 19. Senecio aquaticus. 19.90. S. Jacobaea. Ig.

Senecio Doronicuma $\alpha . \beta$. I08. I 21.

S. erucifolius . 1 о.

S. laciniatus. 96. II I. II2. I 6 .

Serratula tinctoria .90.п Seseli annuum. I9.

S. elatum. 19. 129.

Silene acaulis.98. 101. 105. $S$. acaulis var. $f$. albo.ror. S. Saxifraga . гог. 1о3. 108. 121.129.

Sisymbrium Nasturtium . I9. 90.

Solanum Dulcamara. 19. 90.

S. miniatum . 90. fr. miniat. et fr. lut. I9.

Soldanella montana. $9^{6 .}$ Solidago alpestris varietà della $S$.Virgaurea . I16. Spartium junceum. I9. $S$. radiatum. 129.

Spallanzania Agrimonoides, e suo carattere generico e specifico.96. I22. Sphaeria concentrica, osservazioni microscopiche sulla sua interna tessitura . 90. Spergula saginoides. 98. 1 03 . I 16. 
Sporotrichum aureum.reg. Stachys alpina. II4. 122. Stellaria crassifolia. I20. Sticta syluatica. 97. 107. Stipa pennata. I9. II.

Symphytuintuberosum 123. Tamarix germanica. 129 . L'amus communis. $19.9 \mathbf{I}$. 129.

Tizxus baccata. 107. 120. Teucrium Chamaedrys.rg. 91. 3. 91.129.

Thalictrum minus. Io5.

120. I22.

Thesium alpinum . I22.

Thrincia hirta. 19.

Thymus alpinus 97 105.120. T. Calamintha.r9.9r. 129. T. Nepeta. I9.91. I29.

T. grandiflorus . 91 .

Tilia europaea. I14. 120. I 29.

T. microphylla, II4. I20. I29.

Tofieldia palustris . 98 . I 17.122 .

Tordylium maximum . I9. $9 \mathbf{I}$.

Tormentilla erecta.97. I 6 . Tremella juniperina. 102. Trifoliumalpinum.101.102. Trollius europaeus. 97 . Tuber cibarium.9т. T. rufum . $9 \mathrm{r}$.

Tulostoma boreale . $9 \mathbf{r}$. Tussilago alba. 97. T. discolor. 60. Ior. T. Farfara. I9. II. T. nivea 97 .

T.Petasites. Ig. Ir. Typha minima. 20.
Ulmus campestris var. latifolia. 114. 129. Ulva gelatinosa. I9.

U. turbinata. 19. 44. 45. Uredo aspidii . I I 7 .

Usnea ceratina . 102.

Uvularia amplexifolia 114. Vaccinium Myrtillus. 105. $V$. Vitis Idaea. 105. 108. Valeriana officinalis. Ig. 97.

Valeriana rubra. 19. 92. var. fl. albo. 19 .

$V$. saxatilis', dai nostri botanici presa per la Valeriana celtica. 60. 99 .

IOI. IO2. I29.

$V$. tripteris . $\mathbf{2} 29$.

Vallisneria spiralis . I 9.

Veratrum album . I01, 106. 122.

$V$. nigrum, I01, I06. I12. 122.

Veronica Anagallis. I9.

V. aphylla. 99. 122.

V. Beccabunga. Ig.

$V$. fruticulosa: 122.

V. officinalis . I9. 97 .

$V$. saxatilis . 106.122.

$V$. serpillifolia. 120. 122.

V. spicata. Ig. I 10.

$V$. Teucrium, di cui è varietà la V. latifolia.92. 129. $V$. urticaefolia . 129 .

Viburnum Lantana. Ig.

Vicia lutea. 19. 93.

Viola biflora. 60. 102. I1 $\%$. I22.

V. mirabilis . 93 .

$V$. montana. II 7 .

$V$.tricolor $\alpha . \beta .9^{3.120 .122 .}$ 




